\title{
Solitons and Yukawa couplings in nearly Kähler flux compactifications
}

\author{
Brian P. Dolan ${ }^{1,2, *}$ and Richard J. Szabo ${ }^{3,4, \dagger}$ \\ ${ }^{1}$ Department of Mathematical Physics, National University of Ireland, Maynooth, County Kildare, Ireland \\ ${ }^{2}$ School of Theoretical Physics, Dublin Institute of Advanced Studies, 10 Burlington Road, Dublin 4, Ireland \\ ${ }^{3}$ Department of Mathematics, Heriot-Watt University, Colin Maclaurin Building, Riccarton, Edinburgh EH14 4AS, United Kingdom \\ ${ }^{4}$ Maxwell Institute for Mathematical Sciences, Edinburgh, United Kingdom
}

(Received 3 July 2013; published 5 September 2013)

\begin{abstract}
We study vacuum states and symmetric fermions in the equivariant dimensional reduction of the YangMills-Dirac theory over the six-dimensional homogeneous space $\mathrm{SU}(3) / \mathrm{U}(1) \times \mathrm{U}(1)$ endowed with a family of SU(3) structures including a nearly Kähler structure. We derive the fixed tree-level scalar potentials of the induced Yang-Mills-Higgs theory and compute the dynamically generated gauge and Higgs boson masses as functions of the metric moduli of the coset space. We find an integrable subsector of the Higgs field theory that is governed by a sine-Gordon-type model whose topological soliton solutions are determined nonperturbatively by the gauge coupling and that tunnel between families of infinitely degenerate vacua. The reduction of the Dirac action for symmetric fermions yields exactly massless chiral fermions containing subsectors that have fixed tree-level Yukawa interactions. We compute dynamical fermion mass matrices explicitly and compare them at different points of the moduli space, some of which support consistent heterotic flux vacua.
\end{abstract}

DOI: 10.1103/PhysRevD.88.066002

PACS numbers: 11.25.Mj, 04.50.- h, 11.10.Kk

\section{INTRODUCTION}

Superstring compactifications with fluxes along the internal manifold are believed to provide a means of connecting superstring theory to observable low-energy physics while evading the unfavorable features of the more common Calabi-Yau compactifications. The presence of fluxes deforms the compactification manifold and requires the introduction of non-Kähler geometries in six dimensions [1,2]. In particular, in heterotic string theory the relevant flux is the Neveu-Schwarz 3-form background $H$, which is usually taken as a source for torsion on sixdimensional manifolds with a SU(3) structure. One of the main goals in the study of heterotic string compactifications is to understand how the dimensional reduction of the ten-dimensional $\mathcal{N}=1$ supersymmetric Yang-Mills gauge sector can be used to fix the multitude of free parameters present in the Higgs and Yukawa sectors of the Standard Model, and its extensions.

The pioneering dimensional reduction schemes are the Scherk-Schwarz reduction [3] and coset space dimensional reduction (see [4] for a review). The coset space dimensional reduction of heterotic supergravity over nearly Kähler homogeneous spaces is considered in [5]. Of the four known compact six-dimensional nearly Kähler manifolds, only the flag manifold $\mathbb{F}_{3}:=\mathrm{SU}(3) / \mathrm{U}(1) \times \mathrm{U}(1)$ seems to produce interesting and nontrivial consistent heterotic string vacua; see e.g. [6-8]. The coset space dimensional reduction of the supersymmetric Yang-Mills gauge sector over $\mathbb{F}_{3}$ is considered in e.g. [9]. An alternative

\footnotetext{
*bdolan@thphys.nuim.ie

†R.J.Szabo@hw.ac.uk
}

dimensional reduction scheme over homogeneous spaces is provided by equivariant dimensional reduction (see [10] for reviews); this scheme was applied to the internal space $\mathbb{F}_{3}$ in $[11,12]$.

In this paper we will study the equivariant dimensional reduction of Yang-Mills-Dirac theory over the coset space $\mathbb{F}_{3}$, focusing attention on the Higgs and Yukawa sectors of the induced field theory. Our field theory should be regarded as a toy model, which is the first step in describing the full gauge sector of heterotic string theory, in the sense that it involves two important omissions. First, we start with unitary gauge groups, rather than the desired $\mathrm{E}_{8}$ gauge group of the $\mathcal{N}=1$ supersymmetric gauge theory. Second, we do not demand supersymmetry of our initial Lagrangian. Incorporating both of these restrictions would be an important test of the viability of equivariant dimensional reduction in producing realistic physical relatives of the Standard Model; our preliminary analysis in this paper demonstrates that indeed an interesting vacuum structure and physical masses are induced by this scheme. However, our constructions and results are interesting in their own right, without any reference to heterotic string compactifications, as we now explain.

We consider the most general family of quasi-Kähler $\mathrm{SU}(3)$ structures on $\mathbb{F}_{3}$, one member of which is its standard nearly Kähler structure. This extends the analysis of $[13,14]$, which demonstrates how equivariant dimensional reduction over Kähler coset spaces can yield physical particle spectra that are qualitatively analogous to that of the Standard Model, to non-Kähler compactification manifolds; it extends the considerations of [12] to incorporate symmetric fermions. We will study the vacuum structure of the induced Higgs sector and compute the Higgs and gauge 
boson masses, induced by dynamical symmetry breaking, as functions on the moduli space of SU(3) structures. We will also describe in detail the structure of the Yukawa couplings at various points of the moduli space and compute induced fermion mass matrices explicitly after dynamical symmetry breaking. We will see that the nearly Kähler member of the family of SU(3) structures is naturally singled out, as has been observed previously from considerations based on supersymmetry.

The present paper is structured as follows. In Sec. II we review some standard facts about the geometry of the coset space $\mathbb{F}_{3}$, including the construction of homogeneous gauge fields, a three-parameter family of SU(3) structures, Dirac operators associated with the torsional connections of the SU(3) structures twisted by homogeneous background fields, and the structure of their harmonic spinors. In Sec. III we review the construction of SU(3)-invariant gauge fields on product manifolds $M \times \mathbb{F}_{3}$ associated with irreducible representations of SU(3) and extend the construction to SU(3)-symmetric fermion fields. In Sec. IV we study the vacuum structure of the induced Yang-MillsHiggs theory on $M$ and compute the tree-level Higgs potential and the induced boson masses after spontaneous symmetry breaking. In particular, we identify a subsector of the Higgs field theory that contains infinitely degenerate vacua that are connected by sine-Gordon-type soliton field configurations, and we completely classify the physical field content associated with an arbitrary irreducible SU(3) module. In Sec. V we classify those representations of SU(3) that allow for nontrivial tree-level Yukawa interactions between symmetric fermions in the reduced YangMills-Higgs-Dirac theory on $M$. We show that at the nearly Kähler locus of the moduli space a natural class of Yukawa couplings can be obtained via reduction using constant harmonic spinors on $\mathbb{F}_{3}$, and that nonzero fermion masses are induced by dynamical symmetry breaking. We also compare the Yukawa couplings at other points of the quasi-Kähler moduli space and with those associated with the standard Kähler geometry of the homogeneous space $\mathbb{F}_{3}$. Our results are summarized in the concluding Sec. VI, and finally two appendixes at the end of the paper contain some technical details of the constructions that are used in the main text: In Appendix A we summarize the relevant data for the $\mathrm{SU}(3)$ representations that we use, while in Appendix B we list the SU(3)-invariant field strengths for arbitrary irreducible SU(3) modules.

\section{GEOMETRY OF THE HOMOGENEOUS SPACE SU(3)/U(1) $\times$ U(1)}

The coset space $\mathbb{F}_{3}:=\mathrm{SU}(3) / \mathrm{U}(1) \times \mathrm{U}(1)$ is a reductive but not symmetric homogeneous space. In this section we describe the quasi-Kähler geometry of $\mathbb{F}_{3}$, referring to $[11,12]$ for further details. We will also describe the spin geometry of $\mathbb{F}_{3}$ and the construction of $\mathrm{SU}(3)$-invariant spinor fields.

\section{A. Bimonopole fields}

The projective plane $\mathbb{C} P^{2}$ and the complete flag manifold $\mathbb{F}_{3}$ on $\mathbb{C}^{3}$ are related through the fibrations

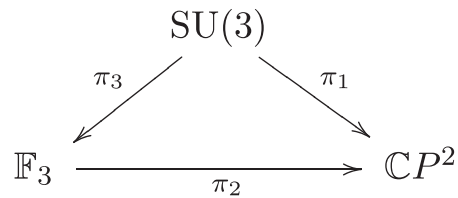

with fibers $\mathrm{U}(1) \times \mathrm{SU}(2), \mathrm{SU}(2) / \mathrm{U}(1)$ and $T:=\mathrm{U}(1) \times$ $\mathrm{U}(1)$ for the bundle projections $\pi_{1}, \pi_{2}$ and $\pi_{3}$, respectively. The $\mathbb{C} P^{1}$-bundle $\pi_{2}$ has structure group SU(2) and describes $\mathbb{F}_{3}$ as the twistor space of $\mathbb{C} P^{2}$. In the following we will exploit this description to construct natural gauge potentials on $\mathbb{F}_{3}$. We could also consider nonmaximal embeddings $\mathrm{U}(1) \times \mathrm{U}(1) \rightarrow \mathrm{SU}(3)$, which are parametrized by a pair of integers $(r, s)$ and lead to quotients $\mathbb{F}_{3} / \mathbb{Z}_{r} \times \mathbb{Z}_{s}$ by freely acting cyclic groups corresponding to inclusion of discrete Wilson line fluxes; the case $(r, s)=$ $(3,1)$ is considered in [9].

With $y^{1}, y^{2}$ local complex coordinates on the base space $\mathbb{C} P^{2}$, we define

$$
\begin{aligned}
T & :=\left(\begin{array}{c}
\bar{y}^{\overline{2}} \\
y^{1}
\end{array}\right), \\
W & :=\gamma \mathbf{1}_{2}-\frac{1}{\gamma+1} T T^{\dagger} \text { and } \\
\gamma & =\sqrt{1+T^{\dagger} T}=\sqrt{1+y^{\alpha} \bar{y}^{\bar{\alpha}}} .
\end{aligned}
$$

Let us introduce one-forms on $\mathbb{C} P^{2}$ given by

$$
\begin{aligned}
& b=\frac{1}{4 \gamma^{2}}\left(T^{\dagger} \mathrm{d} T-\mathrm{d} T^{\dagger} T\right) \quad \text { and } \\
& B=\frac{1}{\gamma^{2}}\left(W \mathrm{~d} W+T \mathrm{~d} T^{\dagger}-\frac{1}{2} \mathrm{~d} T^{\dagger} T-\frac{1}{2} T^{\dagger} \mathrm{d} T\right)
\end{aligned}
$$

together with

$$
\begin{aligned}
\theta & =\left(\begin{array}{l}
\theta^{\overline{2}} \\
\theta^{1}
\end{array}\right):=\frac{2 \Lambda}{\gamma^{2}} W \mathrm{~d} T \\
& =\frac{2 \Lambda}{\gamma}\left(\begin{array}{l}
\mathrm{d} \bar{y}^{\overline{2}} \\
\mathrm{~d} y^{1}
\end{array}\right)-\frac{2 \Lambda}{\gamma^{2}(\gamma+1)}\left(\begin{array}{l}
\bar{y}^{\overline{2}} \\
y^{1}
\end{array}\right)\left(\bar{y}^{\overline{1}} \mathrm{~d} y^{1}+y^{2} \mathrm{~d} \bar{y}^{\overline{2}}\right),
\end{aligned}
$$

where the real parameter $\Lambda$ characterizes the "size" of the base $\mathbb{C} P^{2}$. Here $\theta^{1}$ and $\theta^{2}$ form a local SU(3)-equivariant orthonormal basis of $(1,0)$-forms on $\mathbb{C} P^{2}$, with respect to the natural right isometric action of $G=\mathrm{SU}(3)$ on the coset. In this frame, the one-form $b$ is an anti-self-dual $\mathrm{u}(1)$ connection (monopole potential) on a complex line bundle over $\mathbb{C} P^{2}$, while the gauge potential $B$ is the (canonical) $\mathrm{u}(2)$-valued Levi-Cività connection on the tangent bundle of the coset space $\mathbb{C} P^{2}$. The corresponding field strengths are given by 
$f^{-}:=\mathrm{d} b=\frac{1}{8 \Lambda^{2}} \theta^{\dagger} \wedge \theta=-\frac{1}{8 \Lambda^{2}}\left(\theta^{1} \wedge \theta^{\overline{1}}-\theta^{2} \wedge \theta^{\overline{2}}\right)$

and

$$
\begin{aligned}
F^{+} & :=\mathrm{d} B^{+}+B^{+} \wedge B^{+} \\
& =-\frac{1}{8 \Lambda^{2}}\left(\begin{array}{cc}
\theta^{1} \wedge \theta^{\overline{1}}+\theta^{2} \wedge \theta^{\overline{2}} & 2 \theta^{\overline{1}} \wedge \theta^{\overline{2}} \\
-2 \theta^{1} \wedge \theta^{2} & -\theta^{1} \wedge \theta^{\overline{1}}-\theta^{2} \wedge \theta^{\overline{2}}
\end{array}\right)
\end{aligned}
$$

where

$$
B^{+}=\left(\begin{array}{ll}
a_{+} & -\bar{b}_{+} \\
b_{+} & -a_{+}
\end{array}\right):=B+b \mathbf{1}_{2}
$$

and

$$
F:=\mathrm{d} B+B \wedge B=\frac{1}{4 \Lambda^{2}} \theta \wedge \theta^{\dagger}=F^{+}-f^{-} \mathbf{1}_{2} .
$$

A representative element of the fiber space $\mathbb{C} P^{1} \cong$ $\mathrm{SU}(2) / \mathrm{U}(1) \cong S^{2}$ is a local section of the Hopf fibration $S^{3} \rightarrow S^{2}$ given by the matrix

$$
h=\frac{1}{\sqrt{1+\zeta \bar{\zeta}}}\left(\begin{array}{cc}
1 & -\bar{\zeta} \\
\zeta & 1
\end{array}\right) \in \mathrm{SU}(2) \cong S^{3},
$$

where $\zeta$ is a local complex coordinate on $\mathbb{C} P^{1}$. We may then define one-forms on $\mathbb{F}_{3}$ by the fiberwise gauge transformations

$$
\hat{\theta}=h^{\dagger} \theta=\frac{1}{\sqrt{1+\zeta \bar{\zeta}}}\left(\begin{array}{c}
\theta^{\overline{2}}+\bar{\zeta} \theta^{1} \\
\theta^{1}-\zeta \theta^{\overline{2}}
\end{array}\right)=:\left(\begin{array}{c}
\hat{\theta}^{\overline{2}} \\
\hat{\theta}^{1}
\end{array}\right)
$$

and

$$
\begin{aligned}
\hat{B} & =h^{\dagger} B h+h^{\dagger} \mathrm{d} h=\hat{B}^{+}-b \mathbf{1}_{2} \\
& =:\left(\begin{array}{cc}
\hat{a}_{+} & -\frac{1}{2 R} \hat{\theta}^{\overline{3}} \\
\frac{1}{2 R} \hat{\theta}^{3} & -\hat{a}_{+}
\end{array}\right)-b \mathbf{1}_{2},
\end{aligned}
$$

with

$\hat{a}_{+}=\frac{1}{1+\zeta \bar{\zeta}}\left((1-\zeta \bar{\zeta}) a_{+}+\bar{\zeta} b_{+}-\zeta \bar{b}_{+}+\frac{1}{2}(\bar{\zeta} \mathrm{d} \zeta-\zeta \mathrm{d} \bar{\zeta})\right)$

and

$$
\hat{\theta}^{3}=\frac{2 R}{1+\zeta \bar{\zeta}}\left(\mathrm{d} \zeta+b_{+}-2 \zeta a_{+}+\zeta^{2} \bar{b}_{+}\right) .
$$

Here $b, a_{+}$and $b_{+}$are given by (2.3) and (2.7), while $R$ is the radius of the fiber two-sphere $S^{2} \cong \mathbb{C} P^{1}$. Note that the restriction of the one-form (2.12) to the fiber is the usual Dirac monopole potential on $\mathbb{C} P^{1}$. The corresponding field strengths are given by

$$
\begin{aligned}
\hat{f}^{-} & =f^{-}=\mathrm{d} b=-\frac{1}{8 \Lambda^{2}}\left(\hat{\theta}^{1} \wedge \hat{\theta}^{\overline{1}}-\hat{\theta}^{2} \wedge \hat{\theta}^{\overline{2}}\right) \\
& =-\frac{1}{8 \Lambda^{2}}\left(\theta^{1} \wedge \theta^{\overline{1}}-\theta^{2} \wedge \theta^{\overline{2}}\right)
\end{aligned}
$$

and

$$
\begin{aligned}
\hat{F}^{+} & =\mathrm{d} \hat{B}^{+}+\hat{B}^{+} \wedge \hat{B}^{+} \\
& =-\frac{1}{8 \Lambda^{2}}\left(\begin{array}{cc}
\hat{\theta}^{1} \wedge \hat{\theta}^{\overline{1}}+\hat{\theta}^{2} \wedge \hat{\theta}^{\overline{2}} & 2 \hat{\theta}^{\overline{1}} \wedge \hat{\theta}^{\overline{2}} \\
-2 \hat{\theta}^{1} \wedge \hat{\theta}^{2} & -\hat{\theta}^{1} \wedge \hat{\theta}^{\overline{1}}-\hat{\theta}^{2} \wedge \hat{\theta}^{\overline{2}}
\end{array}\right),
\end{aligned}
$$

together with the Cartan-Maurer equation

$$
\mathrm{d} \hat{\theta}+\left(\hat{B}-2 b \mathbf{1}_{2}\right) \wedge \hat{\theta}=0 .
$$

The gauge fields $\hat{f}^{-}=\pi_{2}^{*} f^{-}$and $\hat{F}^{+}=\pi_{2}^{*} F^{+}$are pullbacks of the monopole and instanton gauge fields $f^{-}$and $F^{+}$on $\mathbb{C} P^{2}$ to the flag manifold $\mathbb{F}_{3}$ by the twistor fibration $\pi_{2}$ from (2.1); in this setting $\mathbb{F}_{3}=\mathbb{C} P(E)$ is the split manifold of the tangent bundle of $\mathbb{C} P^{2}$ [15], regarded as a complex vector bundle $E$ of rank two over $\mathbb{C} P^{2}$ with structure group $\mathrm{U}(2)$. We call the pair $\left(-\hat{a}_{+}, b\right)$ of $\mathrm{u}(1)$-valued gauge potentials on $\mathbb{F}_{3}$ a bimonopole potential; it will play an instrumental role throughout this paper.

\section{B. SU(3) structures}

The metric and a corresponding almost Kähler structure on $\mathbb{F}_{3} \mathrm{read}$

$$
\begin{aligned}
& \hat{\mathrm{g}}=\hat{\theta}^{1} \otimes \hat{\theta}^{\overline{1}}+\hat{\theta}^{2} \otimes \hat{\theta}^{\overline{2}}+\hat{\theta}^{3} \otimes \hat{\theta}^{\overline{3}} \quad \text { and } \\
& \hat{\omega}=\frac{\mathrm{i}}{2}\left(\hat{\theta}^{1} \wedge \hat{\theta}^{\overline{1}}+\hat{\theta}^{2} \wedge \hat{\theta}^{\overline{2}}+\hat{\theta}^{3} \wedge \hat{\theta}^{\overline{3}}\right),
\end{aligned}
$$

where $\hat{\theta}^{\alpha}$ with $\alpha=1,2,3$ are given in (2.10) and (2.13). The SU(3)-invariant one-forms $\hat{\theta}^{\alpha}$ define a compatible integrable almost complex structure $\mathcal{J}_{+}$on $\mathbb{F}_{3}$, i.e. a complex structure, such that $\mathcal{J}_{+} \hat{\theta}^{\alpha}=\mathrm{i} \hat{\theta}^{\alpha}$ so that $\hat{\theta}^{\alpha}$ are $(1,0)$-forms with respect to $\mathcal{J}_{+}$. From (2.14), (2.15), and (2.16) we obtain the Cartan structure equations

$$
\mathrm{d} \hat{\theta}^{\alpha}+\hat{\Gamma}_{\beta}^{\alpha} \wedge \hat{\theta}^{\beta}=0,
$$

which define the Levi-Cività connection

$$
\hat{\Gamma}=\left(\hat{\Gamma}_{\beta}^{\alpha}\right)=\left(\begin{array}{ccc}
-\hat{a}_{+}-3 b & 0 & -\frac{1}{2 R} \hat{\theta}^{\overline{2}} \\
0 & -\hat{a}_{+}+3 b & \frac{1}{2 R} \hat{\theta}^{\overline{1}} \\
\frac{R}{4 \Lambda^{2}} \hat{\theta}^{2} & -\frac{R}{4 \Lambda^{2}} \hat{\theta}^{1} & -2 \hat{a}_{+}
\end{array}\right)
$$

on the tangent bundle of $\mathbb{F}_{3}$. From (2.18) and (2.19) it follows that $\hat{\omega}$ is Kähler, i.e. $\mathrm{d} \hat{\omega}=0$, if and only if

$$
R^{2}=2 \Lambda^{2} .
$$

Then the connection matrix $\hat{\Gamma}$ in (2.19) takes values in the Lie algebra su(3), i.e. the holonomy group is $\mathrm{SU}(3)$. 
Let us now introduce the forms

$$
\Theta^{1}:=\hat{\theta}^{1}, \quad \Theta^{2}:=\hat{\theta}^{2} \quad \text { and } \quad \Theta^{3}:=\hat{\theta}^{\overline{3}},
$$

which are of type $(1,0)$ with respect to an almost complex structure $\mathcal{J}_{-}$, i.e. $\mathcal{J}_{-} \Theta^{\alpha}=\mathrm{i} \Theta^{\alpha}$. The almost complex structure $\mathcal{J}_{-}$is obtained from $\mathcal{J}_{+}$by changing its sign along the $\mathbb{C} P^{1}$ fibers of the twistor bundle $\pi_{2}$, i.e. $\mathcal{J}_{ \pm} \Theta^{1,2}=\mathrm{i} \Theta^{1,2}, \mathcal{J}_{ \pm} \Theta^{3}=\mp \mathrm{i} \Theta^{3}$. It is never integrable; i.e. the corresponding Nijenhuis tensor is nonvanishing. Using the redefinition (2.21), we obtain from (2.18) and (2.19) the Cartan structure equations

$\mathrm{d} \Theta^{\alpha}+\Gamma_{\beta}^{\alpha} \wedge \Theta^{\beta}=H^{\alpha}$ and $\mathrm{d} \Theta^{\bar{\alpha}}+\Gamma_{\bar{\beta}}^{\bar{\alpha}} \wedge \Theta^{\bar{\beta}}=H^{\bar{\alpha}}$,

where the left-hand sides define the (torsional) metric connection

$$
\begin{gathered}
\Gamma=\left(\Gamma_{\beta}^{\alpha}\right)=\left(\begin{array}{ccc}
-\hat{a}_{+}-3 b & 0 & 0 \\
0 & -\hat{a}_{+}+3 b & 0 \\
0 & 0 & 2 \hat{a}_{+}
\end{array}\right) \text {and } \\
\Gamma_{\bar{\beta}}^{\bar{\alpha}}=-\Gamma_{\beta}^{\alpha}
\end{gathered}
$$

with holonomy $\mathrm{U}(1) \times \mathrm{U}(1) \subset \mathrm{SU}(3)$, while the righthand sides define the Nijenhuis tensor (torsion) with components $H_{\bar{\beta} \bar{\gamma}}^{\alpha}$ given by

$$
\left(H^{\alpha}\right)=\left(\frac{1}{2} H_{\bar{\beta} \bar{\gamma}}^{\alpha} \Theta^{\bar{\beta}} \wedge \Theta^{\bar{\gamma}}\right)=\frac{1}{2 R}\left(\begin{array}{c}
\Theta^{\overline{2}} \wedge \Theta^{\overline{3}} \\
\Theta^{\overline{3}} \wedge \Theta^{\overline{1}} \\
\frac{R^{2}}{\Lambda^{2}} \Theta^{\overline{1}} \wedge \Theta^{\overline{2}}
\end{array}\right) .
$$

We also have

$$
\mathrm{d} b=-\frac{1}{8 \Lambda^{2}}\left(\Theta^{1} \wedge \Theta^{\overline{1}}-\Theta^{2} \wedge \Theta^{\overline{2}}\right)
$$

and

$$
\mathrm{d} \hat{a}_{+}=-\frac{1}{8 \Lambda^{2}}\left(\Theta^{1} \wedge \Theta^{\overline{1}}+\Theta^{2} \wedge \Theta^{\overline{2}}\right)+\frac{1}{4 R^{2}} \Theta^{3} \wedge \Theta^{\overline{3}}
$$

for the Abelian gauge fields on $\mathbb{F}_{3}$.

The pair of forms $(\omega, \Omega)$ given by

$$
\begin{aligned}
& \omega=\frac{\mathrm{i}}{2}\left(\Theta^{1} \wedge \Theta^{\overline{1}}+\Theta^{2} \wedge \Theta^{\overline{2}}+\Theta^{3} \wedge \Theta^{\overline{3}}\right) \text { and } \\
& \Omega=\Theta^{1} \wedge \Theta^{2} \wedge \Theta^{3}
\end{aligned}
$$

defines a one-parameter family of invariant SU(3) structures on $\mathbb{F}_{3}$, parametrized by the ratio $\frac{R^{2}}{\Lambda^{2}}$. From (2.22), (2.23), and (2.24) it follows that the homogeneous manifold $\mathbb{F}_{3}$ is nearly Kähler, i.e. $\mathrm{d} \omega=\frac{3}{2} W_{1} \operatorname{Im} \Omega$ and $\mathrm{d} \Omega=$ $W_{1} \omega \wedge \omega$ with $W_{1} \in \mathbb{R}$, if and only if

$$
R^{2}=\Lambda^{2},
$$

in which case $W_{1}=\frac{1}{R}$. In this instance we will fix the scales of the fiber $\mathbb{C} P^{1}$ and the base $\mathbb{C} P^{2}$ in $\mathbb{F}_{3}$ as

$$
R=\Lambda=\sqrt{3},
$$

in order that the connection $\Gamma$ in (2.23) coincides with the canonical connection on the principal torus bundle $\pi_{3}$ from (2.1). The $(1,1)$-form $\omega$ is almost Kähler for the metric

$$
g=\Theta^{1} \otimes \Theta^{\overline{1}}+\Theta^{2} \otimes \Theta^{\overline{2}}+\Theta^{3} \otimes \Theta^{\overline{3}} .
$$

We obtain from the nearly Kähler structure a threeparameter family of invariant quasi-Kähler SU(3) structures by rescaling the one-forms $\Theta^{\alpha}$ by constant metric moduli $\boldsymbol{s}_{\alpha} \in \mathbb{R}$ as

$$
\Theta^{\alpha} \mapsto \tilde{\Theta}^{\alpha}=\frac{1}{2 \sqrt{3}} \boldsymbol{s}_{\alpha}^{-1} \Theta^{\alpha}
$$

for $\alpha=1,2,3$. The metric and the fundamental two-form become

$$
\begin{aligned}
& \tilde{\mathrm{g}}=\tilde{\Theta}^{1} \otimes \tilde{\Theta}^{\overline{1}}+\tilde{\Theta}^{2} \otimes \tilde{\Theta}^{\overline{2}}+\tilde{\Theta}^{3} \otimes \tilde{\Theta}^{\overline{3}} \text { and } \\
& \tilde{\omega}=\frac{\mathrm{i}}{2}\left(\tilde{\Theta}^{1} \wedge \tilde{\Theta}^{\overline{1}}+\tilde{\Theta}^{2} \wedge \tilde{\Theta}^{\overline{2}}+\tilde{\Theta}^{3} \wedge \tilde{\Theta}^{\overline{3}}\right) .
\end{aligned}
$$

The 3-form $\mathrm{d} \tilde{\omega}$ has only $(3,0)$ and $(0,3)$ components with respect to the almost complex structure $\mathcal{J}_{-}$; i.e. $\tilde{\omega}$ is quasiKähler. The associated family of connections $\tilde{\Gamma}$ on the tangent bundle $T \mathbb{F}_{3}$ corresponds to different regularization schemes that are related by field redefinitions of the underlying world sheet sigma model in heterotic string theory. In particular, at the nearly Kähler locus $\varsigma_{1}=\varsigma_{2}=\varsigma_{3}=\frac{1}{2 R}$ of the moduli space we can restore the fiber $\mathbb{C} P^{1}$ radius $R$, while for $\varsigma_{1}=\varsigma_{2}=\frac{1}{2 \Lambda}, \varsigma_{3}=\frac{1}{2 R}$ we can restore both of our original base and fiber size parameters $\Lambda$ and $R$, with the Kähler locus in the moduli space given by (2.20). Notice that the Kähler and nearly Kähler loci correspond not only to different choices of almost complex structures $\mathcal{J}_{+}$and $\mathcal{J}_{-}$on $\mathbb{F}_{3}$ but also to metrics $\mathrm{g}$ and $\mathrm{g}$, which differ by a factor of 2 along the fiber direction $\mathbb{C} P^{1} \hookrightarrow \mathbb{F}_{3}$.

The generic case of an $\mathrm{SU}(3)$ structure is classified by intrinsic torsion [16], which can be characterized by the decomposition of the torsion $H$ into irreducible $\mathrm{SU}(3)$ modules (see Appendix A); they are referred to as the five torsion classes $W_{1}, \ldots, W_{5}$. For an almost Kähler manifold all five intrinsic torsion classes can be generically nonvanishing. For a Kähler manifold $H \in W_{5}=$ $\hat{V}^{1,0} \oplus \hat{V}^{0,1}$, while in the nearly Kähler case $H \in W_{1}=$ $\hat{V}^{0,0} \oplus \hat{V}^{0,0}$. Quasi-Kähler structures have $H \in W_{1} \oplus W_{2}$. Calabi-Yau manifolds correspond to the vanishing of all five intrinsic torsion classes.

\section{Dirac operators with torsion}

The homogeneous space $\mathbb{F}_{3}$ is a spin manifold with an $\mathrm{SU}(3)$-invariant spin structure. Its main feature as a sixdimensional SU(3)-structure manifold is that its first Chern 
class vanishes and the canonical bundle is trivial. This implies the existence of a metric connection (the canonical connection) with totally skew-symmetric torsion and holonomy contained in $G=\mathrm{SU}(3)$, which admits a covariantly constant spinor without coupling to gauge fields. We will describe invariant fermion fields via the index theorem for the twisted Dirac operator on $\mathbb{F}_{3}$ corresponding to these torsional connections, which gives the chiral asymmetry of zero modes of the Dirac operator. Torsion does not affect the principal symbol of the Dirac operator; hence the index is the same at every point in the moduli space; the torsion part can be regarded as a continuous deformation of the Dirac operator constructed from the Levi-Cività spin connection, and the index is invariant under compact perturbations. Nevertheless, the presence of intrinsic torsion can affect the fermion counting, as fermions of different chirality can become intertwined and the usual chirality operator cannot be used to define the index.

In our three-parameter family of quasi-Kähler $\mathrm{SU}(3)$ structures, we choose a basis of $8 \times 8 \gamma$ matrices $\tilde{\gamma}^{\alpha}, \tilde{\gamma}^{\bar{\alpha}}=$ $\left(\tilde{\gamma}^{\alpha}\right)^{\dagger}$ for the Clifford algebra on $\mathbb{F}_{3}$ compatible with the metric $\tilde{\mathrm{g}}$ in (2.32) and the orthonormal one-forms $\tilde{\Theta}^{\alpha}, \tilde{\Theta}^{\bar{\alpha}}$. In this basis the Clifford relations read

$\left\{\tilde{\gamma}^{\alpha}, \tilde{\gamma}^{\bar{\beta}}\right\}=\delta^{\alpha \beta} \mathbf{1}_{8} \quad$ and $\quad\left\{\tilde{\gamma}^{\alpha}, \tilde{\gamma}^{\beta}\right\}=0=\left\{\tilde{\gamma}^{\bar{\alpha}}, \tilde{\gamma}^{\bar{\beta}}\right\}$

with $\alpha, \beta=1,2,3$ (complex) orthonormal indices with respect to the metric $\tilde{\mathrm{g}}$. The map identifying differential forms on $\mathbb{F}_{3}$ with elements of the Clifford algebra is given by

$$
\begin{aligned}
\eta & =\eta_{\alpha_{1} \ldots \alpha_{r} \bar{\beta}_{1} \ldots \bar{\beta}_{s}} \tilde{\Theta}^{\alpha_{1}} \wedge \ldots \wedge \tilde{\Theta}^{\alpha_{r}} \wedge \tilde{\Theta}^{\bar{\beta}_{1}} \wedge \ldots \wedge \tilde{\Theta}^{\bar{\beta}_{s}} \\
\mapsto \eta & =\eta_{\alpha_{1} \ldots \alpha_{r} \bar{\beta}_{1} \ldots \bar{\beta}_{s}} \tilde{\gamma}^{\left[\alpha_{1}\right.} \ldots \tilde{\gamma}^{\alpha_{r}} \tilde{\gamma}^{\bar{\beta}_{1}} \ldots \tilde{\gamma}^{\left.\bar{\beta}_{s}\right]},
\end{aligned}
$$

where the square brackets denote antisymmetrization over all indices with weight one; this map defines the $G$-equivariant Clifford module $\Lambda^{\bullet} T^{*} \mathbb{F}_{3}$.

SU(3)-equivariant bundles over the coset space $\mathbb{F}_{3}$ are homogeneous vector bundles induced by representations of the maximal torus $T=\mathrm{U}(1) \times \mathrm{U}(1)$ in $\mathrm{SU}(3)$; they are parametrized by charges $(q, m)_{n} \in W^{k, l} \subset \mathbb{Z}^{2}$, which lie in the weight lattices of irreducible representations $\hat{V}^{k, l}$ of SU(3) (see Appendix A). Every such bundle is thus a sum of line bundles of the form $\mathcal{O}_{\mathbb{E}_{3}}(q, m):=\left(\mathcal{L}_{(1)}\right)^{\otimes q} \otimes$ $\left(\mathcal{L}_{(2)}\right)^{\otimes m}$, where the line bundles $\mathcal{L}_{(i)} \rightarrow \mathbb{F}_{3}$ for $i=1,2$ correspond to the generators of $\mathrm{H}^{2}\left(\mathbb{F}_{3} ; \mathbb{Z}\right)=\mathbb{Z} \oplus \mathbb{Z}$; the fiber restriction $\left.\mathcal{L}_{(1)}\right|_{\mathbb{C} P^{1}}$ is the Dirac monopole line bundle corresponding to the generator of $\mathrm{H}^{2}\left(\mathbb{C} P^{1} ; \mathbb{Z}\right)=\mathbb{Z}$, while $\mathcal{L}_{(2)}$ is the pullback by the twistor fibration $\pi_{2}$ from (2.1) of the monopole line bundle corresponding to the generator of $\mathrm{H}^{2}\left(\mathbb{C} P^{2} ; \mathbb{Z}\right)=\mathbb{Z}$. The Dirac operator acting on eightcomponent spinor fields on $\mathbb{F}_{3}$, in the background bimonopole field corresponding to weight vector $(q, m)_{n} \in W^{k, l}$, is given by

$$
\not D_{q, m}=\not \mathbb{F}_{\mathbb{F}_{3}}^{\sigma}+m \not b-q \hat{a}_{+} .
$$

Here $\not_{\mathbb{F}_{3}}^{\sigma}$ is the Dirac operator on $\mathbb{F}_{3}$ involving only the (torsional) spin connection; it can be written as

$$
\not_{\mathbb{F}_{3}}^{\sigma}=\not \partial_{\mathbb{F}_{3}}^{0}-\frac{3}{2} i \sigma H,
$$

where $\not_{\mathbb{F}_{3}}^{0}$ is the Dirac operator associated with the (torsion-free) Levi-Cività connection for the metric $\tilde{\mathrm{g}}$, while $H=\sqrt{3} \operatorname{Im} \Omega$ is the skew-symmetric torsion 3-form of the canonical connection on $\mathbb{F}_{3}$ and we have used the structure constants (A6); the intrinsic torsion of the coset space $\mathbb{F}_{3}$ can be identified with the 3 -form $H$ flux of heterotic supergravity. The real parameter

$$
\sigma=\frac{1}{24 \sqrt{3}}\left(\mathrm{~s}_{1} \varsigma_{2} \varsigma_{3}\right)^{-1}
$$

formally interpolates between the Dirac operator corresponding to the canonical connection (2.23) at $\sigma=1$ and the Levi-Cività connection at $\sigma=0$. The former limit defines a surface of quasi-Kähler structures in the moduli space that contains the nearly Kähler point $\boldsymbol{\varsigma}_{\alpha}=\frac{1}{2 \sqrt{3}}, \alpha=$ $1,2,3$, whereas the latter limit cannot be reached by any continuous variation of the metric moduli. The oneparameter family of Dirac operators (2.36) is a special subclass of the more general families studied in [17] (see also [18]). The volume form $\tilde{\omega}^{\wedge 3} / 3$ ! determines a $\mathbb{Z}_{2}$ grading of the eight-dimensional spinor module $\Delta_{\mathbb{F}_{3}}=$ $\Delta_{\mathbb{F}_{3}}^{+} \oplus \Delta_{\mathbb{F}_{3}}^{-}$on $\mathbb{F}_{3}$ such that the chirality operator

$$
\tilde{\gamma}:=\left[\tilde{\gamma}^{1}, \tilde{\gamma}^{\overline{1}}\right]\left[\tilde{\gamma}^{2}, \tilde{\gamma}^{\overline{2}}\right]\left[\tilde{\gamma}^{3}, \tilde{\gamma}^{\overline{3}}\right]
$$

acts as multiplication by \pm 1 on $\Delta_{\mathbb{F}_{3}}^{ \pm}$. In a suitable basis for the Clifford algebra, the operator (2.35) correspondingly has a chiral decomposition

$$
\not D_{q, m}=\left(\begin{array}{cc}
0 & \not_{q, m}^{+} \\
-\not D_{q, m}^{-} & 0
\end{array}\right),
$$

with the twisted Dolbeault-Dirac operators $\not D_{q, m}^{ \pm}$acting on four-component positive/negative chirality spinor fields on $\mathbb{F}_{3}$.

The (reduced) K-theory of the homogeneous space $\mathbb{F}_{3}$ is generated by the two monopole line bundles $\mathcal{L}_{(i)} \rightarrow \mathbb{F}_{3}$ together with $\mathcal{L}_{(i)} \otimes \mathcal{L}_{(2)}$ and $\mathcal{L}_{(1)} \otimes \mathcal{L}_{(2)} \otimes \mathcal{L}_{(2)}$ for $i=$ 1,2 . The index of the Dirac operator (2.35) is computed by the Atiyah-Singer index theorem

$$
\begin{aligned}
\nu_{q, m} & :=\operatorname{index}\left(i \not D_{q, m}\right) \\
& =\int_{\mathbb{F}_{3}} \operatorname{ch}\left(\left(\mathcal{L}_{(1)}\right)^{\otimes q} \otimes\left(\mathcal{L}_{(2)}\right)^{\otimes m}\right) \wedge \hat{A}\left(\mathbb{F}_{3}\right) \\
& =\int_{\mathbb{F}_{3}}\left(\frac{1}{6} f_{q, m} \wedge f_{q, m} \wedge f_{q, m}-\frac{1}{24} f_{q, m} \wedge p_{1}\left(T \mathbb{F}_{3}\right)\right),
\end{aligned}
$$


where

$$
f_{q, m}=m \mathrm{~d} b-q \mathrm{~d} \hat{a}_{+}
$$

is the curvature of the bimonopole line bundle $\mathcal{O}_{\mathbb{F}_{3}}(q, m)$. For $\mathbb{F}_{3}$ the first Pontrjagin class of the tangent bundle vanishes, $p_{1}\left(T \mathbb{F}_{3}\right)=0$, and using (2.25) and (2.26) we find explicitly $[11,19]$

$$
\nu_{q, m}=\frac{1}{8} q\left(m^{2}-q^{2}\right) .
$$

The index $\nu_{q, m}$ is an integer since $q$ and $m$ have the same parity for $(q, m)_{n} \in W^{k, l}$.

\section{Harmonic spinors}

For each weight $(q, m)_{n} \in W^{k, l}$, the vector space $\operatorname{ker}\left(\not D_{q, m}\right)$ of harmonic spinors on $\mathbb{F}_{3}$ is also independent of the choice of connection on the tangent bundle; the irreducible $\mathrm{SU}(3)$ representation $\hat{V}^{k^{\prime}, l^{\prime}}$ isomorphic to $\operatorname{ker}\left(\not D_{q, m}\right)$, when nonzero, is described in e.g. ([20], Thm. 8.4). However, this is not true in general of the chiral subspaces $\operatorname{ker}\left(\not \not_{q, m}^{ \pm}\right)$. There are two members of the family of Dirac operators (2.36) where the SU(3)-module structure of these subspaces is also known explicitly.

$\sigma=1$. - The Dirac spectrum was computed in [19] from the canonical spin connection on $\mathbb{F}_{3}$ with torsion associated with (2.23); in this case the four operators occurring in the Weitzenböck formula for $\left(\mathrm{i} \not \not_{q, m}\right)^{2}$ mutually commute and hence simultaneously diagonalize to quadratic Casimir invariants for the Lie algebras su(3) and $\mathrm{u}(1) \oplus \mathrm{u}(1)$ [see also ([17], Prop. 3.4)]. For $q<0$ the chiral case $\operatorname{ker}\left(\not \not_{q, m}^{-}\right)=\{0\}$ corresponds to background gauge field configurations on $\mathbb{F}_{3}$ with $q^{2} \geq m^{2}$, for which $\operatorname{ker}\left(\not D_{q, m}^{+}\right)$is isomorphic to the $\mathrm{SU}(3)$-module $\hat{V}^{k^{\prime}, l^{\prime}}$ having $|q|=k^{\prime}+l^{\prime},|m|=k^{\prime}-l^{\prime}$. The corresponding dimension $d^{k^{\prime}, l^{\prime}}$ from (A10) coincides with the index (2.42) after shifting $q \rightarrow q \pm 2$. The antichiral case $\operatorname{ker}\left(\not \not_{q, m}^{+}\right)=\{0\}$ corresponds to $q^{2} \leq m^{2}$, for which the Dirac kernel $\operatorname{ker}\left(\not D_{q, m}^{-}\right)$is isomorphic to the $\mathrm{SU}(3)$ representation $\hat{V}^{k^{\prime}, l^{\prime}}$ with $|q|=k^{\prime},|m|=k^{\prime}+2 l^{\prime}$. The corresponding dimension $d^{k^{\prime}, l^{\prime}}$ agrees with minus the index (2.42) after the shifts $q \rightarrow q \pm 1, m \rightarrow m \pm 3$. In both cases the charge shifts account for the contributions of the intrinsic spin of the fermions to the $\mathrm{U}(1)$ monopole charges. A similar description applies to the $q>0$ case, with chiral and antichiral interchanged. On the other hand, for $q=0$ the index (2.42) is zero and it is possible for an equal number of chiral and antichiral harmonic spinors to coexist. We correspondingly decompose the set of weights $W^{k, l}$ into disjoint subsets

$W_{+}^{k, l}=\left\{(q, m)_{n} \in W^{k, l} \mid m^{2}>q^{2}, q>0\right.$ or $\left.m^{2}<q^{2}, q<0\right\}$,

$W_{-}^{k, l}=\left\{(q, m)_{n} \in W^{k, l} \mid m^{2}<q^{2}, q>0\right.$ or $\left.m^{2}>q^{2}, q<0\right\}$,

$W_{0}^{k, l}=\left\{(q, m)_{n} \in W^{k, l} \mid m^{2}=q^{2}\right.$ or $\left.q=0\right\}$.
Note that for some choices of $(k, l)$, the index vanishes on all weights $(q, m)_{n} \in W^{k, l}$, i.e. $W^{k, l}=W_{0}^{k, l}$. For example, the fundamental representation with $(k, l)=(1,0)$ has weights $(q, m)_{n}=(1,1)_{1}, \quad(-1,1)_{1}$ and $(0,-2)_{0}$, for each of which either $q=0$ or $q^{2}=m^{2}$ and the index (2.42) evaluates to $\nu_{q, m}=0$. This also happens for the six-dimensional representation of $\mathrm{SU}(3)$ with $(k, l)=$ $(2,0)$. On the other hand, there are zero eigenspinors of the Dirac operator for the adjoint representation with $(k, l)=(1,1)$ and for the ten-dimensional decuplet representation with $(k, l)=(3,0)$. The canonical connection naturally appears in the supersymmetry condition for heterotic supergravity.

$\sigma=\frac{1}{3}$. - The operator $\not_{\mathbb{F}_{3}}^{1 / 3}$ is a geometric realization of Kostant's cubic Dirac operator (see e.g. $[17,20]$ ) for the coset $G / T$, whose square may be expressed in a simple way in terms of Casimir operators and scalars alone. When restricted to an irreducible representation of $T$, the eigenvalue of $\left(i \not \mathbb{F}_{3} / 3\right)^{2}$ is the difference of quadratic Casimir invariants for the Lie algebras $\operatorname{su}(3)$ and $u(1) \oplus u(1)$. Moreover, $\not \mathscr{E}_{3}^{1 / 3}$ is the unique Dirac operator, which also respects the sign decomposition in the homogeneous Weyl formula for $G=\mathrm{SU}(3)$, and hence the $\mathrm{SU}(3)$ representation $\hat{V}^{k^{\prime}, l^{\prime}}$ isomorphic to $\operatorname{ker}\left(\not D_{q, m}\right)$ in this case can occur in just one of the chiral subspaces $\operatorname{ker}\left(\not \not_{q, m}^{ \pm}\right)$. From these facts one may identify the vector spaces $\operatorname{ker}\left(\not D_{q, m}^{ \pm}\right)$with irreducible SU(3) modules of dimension given by the index (2.42), analogously to the $\sigma=1$ case above. The appearance of the cubic Dirac operator in the gaugino Dirac equation of heterotic supergravity was observed in [21], as was the completing of squares in the Weitzenböck formula for $\left(\mathrm{i} \phi_{\mathbb{F}_{3}}^{1 / 3}\right)^{2}$ (see also $[18,22,23]$ ).

$\sigma \in \mathbb{R}$.-For parameter values (2.37) that represent generic points in the moduli space, a type of Parthasarathy formula for $\left(\mathrm{i} \phi_{\mathbb{F}_{3}}^{\sigma}\right)^{2}$ involving invariant operators on the coset $G / T$ is derived in ([17], Thm. 3.2); its form is rather complicated and does not seem to provide any useful information about the kernels $\operatorname{ker}\left(\not \not_{q, m}^{ \pm}\right)$beyond the cases $\sigma=1$ and $\sigma=\frac{1}{3}$ above. For simplicity we will for the most part assume that the total number of zero eigenspinors of $\not D_{q, m}$ for a given weight $(q, m)_{n} \in W^{k, l}$ with $\nu_{q, m} \neq 0$ is the same as the index, i.e. $\left|\nu_{q, m}\right|=\operatorname{dim} \operatorname{ker}\left(\not D_{q, m}\right)$, but this need not be true. In the generic quasi-Kähler case there are two free parameters (apart from an overall scale), and it could happen that extra zero modes emerge at specific parameter values. Although the index of the Dirac operator (2.42) cannot change, being a topological invariant, it is possible for an equal number of positive and negative chirality states to appear or disappear at some points $\boldsymbol{s}_{r}$ of the moduli space without changing the value $\nu_{q, m}$. If this happens, then some of the summation formulas of Secs. III B and V below have to be extended to incorporate any extra eigenspinors; while these extra zero modes come 
in SU(3) representations, they cannot have weights in $W^{k, l}$. For simplicity we shall ignore this complication here and assume that there are no such extra spinor harmonics; amongst other things this will simplify some of the notation in already cumbersome formulas. The same convention will be applied for the most part to the possible states with a vanishing index.

We fix a basis of chiral/antichiral harmonic spinors $\chi_{q, m ; \ell}^{ \pm} \in \operatorname{ker}\left(\not D_{q, m}^{ \pm}\right), \ell=1, \ldots,\left|\nu_{q, m}\right|$, on $\mathbb{F}_{3}$ for each weight $(q, m)_{n} \in W_{ \pm}^{k, l}$. They may be chosen to be orthogonal and normalized such that

$$
\begin{aligned}
& \int_{\mathbb{F}_{3}} \frac{\tilde{\omega}^{\wedge 3}}{3 !} \chi_{q, m ; \ell^{\prime}}^{ \pm} \chi_{q, m ; \ell}^{ \pm}=\delta_{\ell, \ell^{\prime}} \quad \text { and } \\
& \int_{\mathbb{F}_{3}} \frac{\tilde{\omega}^{\wedge 3}}{3 !} \chi_{q, m ; \ell^{\prime}}^{\dagger} \chi_{q, m ; \ell}^{ \pm}=0,
\end{aligned}
$$

where the second equality follows from $W_{+}^{k, l} \cap W_{-}^{k, l}=\emptyset$. Note the vector space $\operatorname{ker}\left(\not \not_{q, m}\right)$ of harmonic spinors is independent of the multiplicity label $n$ of the weight state $(q, m)_{n}$.

To explicitly construct the spinor harmonics, following [14] we associate the Clifford generators $\tilde{\gamma}^{\bar{\alpha}}$ and $\tilde{\gamma}^{\alpha}$, respectively, with creation and annihilation operators acting on a fermionic Fock space with vacuum vector $|\Omega\rangle$ such that $\tilde{\gamma}^{\alpha}|\Omega\rangle=0$ for $\alpha=1,2$, 3. A general Fock space state

$$
\begin{aligned}
\chi(z, \bar{z}):= & \chi_{0}(z, \bar{z}) \otimes|\Omega\rangle+\chi_{\bar{\alpha}}(z, \bar{z}) \otimes \tilde{\gamma}^{\bar{\alpha}}|\Omega\rangle \\
& +\frac{1}{2} \chi_{\bar{\alpha} \bar{\beta}}(z, \bar{z}) \otimes \tilde{\gamma}^{\bar{\alpha}} \tilde{\gamma}^{\bar{\beta}}|\Omega\rangle \\
& +\frac{1}{6} \chi_{\bar{\alpha} \bar{\beta} \bar{\rho}}(z, \bar{z}) \otimes \tilde{\gamma}^{\bar{\alpha}} \tilde{\gamma}^{\bar{\beta}} \tilde{\gamma}^{\bar{\rho}}|\Omega\rangle
\end{aligned}
$$

corresponds to a Dirac spinor field on $\mathbb{F}_{3}$, where $z^{\alpha}, \bar{z}^{\bar{\alpha}}$ denote local complex coordinates on $\mathbb{F}_{3}$ and the component of $\chi(z, \bar{z})$ obtained from $k$ creation operators is a $(0, k)$-form. This identifies the space of spinors on $\mathbb{F}_{3}$ with the space of anti-pseudo-holomorphic differential forms $\Lambda^{0, \bullet} T^{*} \mathbb{F}_{3}$. The chirality operator on $\mathbb{F}_{3}$ is given by (2.38), and we see that $\chi_{0}$ and $\chi_{\bar{\alpha}} \bar{\beta}$ are the four components of a positive chirality spinor, while $\chi_{\bar{\alpha}}$ and $\chi_{\overline{1}} \overline{2} \overline{3}$ are the four components of a negative chirality spinor.

\section{EQUIVARIANT DIMENSIONAL REDUCTION OVER SU(3)/U(1) $\times \mathbf{U}(1)$}

In this section we describe the reduction of SU(3)-equivariant vector bundles with connection over the flag manifold $\mathbb{F}_{3}$, again referring to $[11,12]$ for further details. We also present the $\mathrm{SU}(3)$-equivariant dimensional reduction of twisted spinor fields over $\mathbb{F}_{3}$.

\section{A. Invariant gauge fields}

We are interested in the structure of $G$-equivariant gauge fields on manifolds of the form

$$
\mathcal{M}:=M \times \mathbb{F}_{3},
$$

where $M$ is a closed oriented manifold of dimension $d$; the group $G=\mathrm{SU}(3)$ acts trivially on $M$ and in the standard way by isometries of the coset space $\mathbb{F}_{3}=G / T$. By standard induction and reduction, there is an equivalence between smooth $G$-equivariant vector bundles $\mathcal{E}$ over $\mathcal{M}$ and smooth $T$-equivariant vector bundles $E$ over $M$, where the maximal torus $T=\mathrm{U}(1) \times \mathrm{U}(1)$ of $\mathrm{SU}(3)$ acts trivially on $M$. Let $\mathcal{E}^{k, l} \rightarrow \mathcal{M}$ be a rank $N$ Hermitian vector bundle over the space (3.1), associated with an irreducible representation $\hat{V}^{k, l}$ of $\mathrm{SU}(3)$, with structure group $\mathrm{U}(N)$. Given a $T$-equivariant bundle $E^{k, l} \rightarrow M$ of rank $N$ associated with the representation $\left.\hat{V}^{k, l}\right|_{T}$ of $T$, the corresponding $G$-equivariant bundle over $\mathcal{M}$ is defined by induction as

$$
\mathcal{E}^{k, l}=G \times_{T} E^{k, l} .
$$

The action of the maximal torus $T$ on $E^{k, l}$ is defined by the isotopical decomposition

$$
\begin{aligned}
E^{k, l} & =\bigoplus_{(q, m)_{n} \in W^{k, l}} E_{(q, m)_{n}} \otimes V_{(q, m)_{n}} \quad \text { with } \\
E_{(q, m)_{n}} & =\operatorname{Hom}_{T}\left(V_{(q, m)_{n}}, E^{k, l}\right),
\end{aligned}
$$

where $V_{(q, m)_{n}} \cong \mathbb{C}$ are the irreducible $T$ modules occurring in the decomposition of the restriction $\left.\hat{V}^{k, l}\right|_{T}$ and as before $W^{k, l} \subset \mathbb{Z}^{2}$ is the set of weights for the irreducible $\mathrm{SU}(3)$ representation $\hat{V}^{k, l}$ (see Appendix A). The vector bundles $E_{(q, m)_{n}} \rightarrow M$ have rank $N_{(q, m)_{n}}$ and trivial $T$ actions. The rank $N$ of $E^{k, l}$ is given by

$$
N=\sum_{(q, m)_{n} \in W^{k, l}} N_{(q, m)_{n}} .
$$

The action of the $\mathrm{SU}(3)$ operators $I_{\alpha}^{-}, I_{\bar{\alpha}}^{+}$for $\alpha=1,2,3$ is implemented by means of bifundamental Higgs fields

$$
\begin{aligned}
\phi_{(q, m)_{n}}^{1}{ }^{( \pm)} & \in \operatorname{Hom}\left(E_{(q, m)_{n}}, E_{(q-1, m+3)_{n \pm 1}}\right), \\
\phi_{(q, m)_{n}}^{2}{ }^{( \pm)} & \in \operatorname{Hom}\left(E_{(q, m)_{n}}, E_{(q-1, m-3)_{n \pm 1}}\right), \quad \text { and } \\
\phi_{(q, m)_{n}}^{3} & \in \operatorname{Hom}\left(E_{(q, m)_{n}}, E_{(q+2, m)_{n}}\right)
\end{aligned}
$$

together with their adjoints. These bundle morphisms realize the $G$ action of the coset generators, which twists the naive dimensional reduction by "off-diagonal" terms. This construction defines a representation of the quiver $Q^{k, l}$ with vertex set $W^{k, l}$ and arrow set $I_{\alpha}^{-}, \alpha=1,2,3$, whose underlying lattice is just the weight diagram of the representation $\hat{V}^{k, l}$; it explicitly breaks the gauge group of the bundle $E^{k, l}$ as

$$
\mathrm{U}(N) \rightarrow \prod_{(q, m)_{n} \in W^{k, l}} \mathrm{U}\left(N_{(q, m)_{n}}\right) .
$$

The structure group of the principal bundle associated with (3.2) is then 


$$
\mathrm{U}(1) \times \mathrm{U}(1) \times \prod_{(q, m)_{n} \in W^{k, l}} \mathrm{U}\left(N_{(q, m)_{n}}\right) .
$$

The generic form of a $G$-equivariant connection oneform $\mathcal{A}$ on the vector bundle $\mathcal{E}^{k, l} \rightarrow \mathcal{M}$ is determined by specifying a unitary connection $A^{(q, m)_{n}}$ on each bundle $E_{(q, m)_{n}}$. Let $\hat{\Pi}_{(q, m)_{n}}$ be the Hermitian projection of the $T$ restriction of $\hat{V}^{k, l}$ onto the one-dimensional representation of $T=\mathrm{U}(1) \times \mathrm{U}(1)$ with weight vector $(q, m)_{n} \in W^{k, l}$, and let $\Pi_{(q, m)_{n}}$ be the Hermitian projection onto the subbundle $E_{(q, m)_{n}}$ of the bundle (3.3) over $M$. Then an SU(3)equivariant gauge connection $\mathcal{A}$ on the corresponding bundle (3.2) over $\mathcal{M}$ is given by

$$
\begin{aligned}
\mathcal{A}= & \sum_{(q, m)_{n} \in W^{k, l}}\left[A^{(q, m)_{n}} \otimes \hat{\Pi}_{(q, m)_{n}}+\Pi_{(q, m)_{n}} \otimes\left(m b-q \hat{a}_{+}\right) \hat{\Pi}_{(q, m)_{n}}+2 \sqrt{3} \sum_{ \pm} \varsigma_{1}\left(\phi_{(q, m)_{n}}^{1}{ }^{( \pm) \dagger} \otimes \hat{\Pi}_{(q, m)_{n}} I_{1}^{-} \hat{\Pi}_{(q-1, m+3)_{n \pm 1}} \tilde{\Theta}^{1}\right.\right. \\
& \left.+\phi_{(q, m)_{n}}^{1}{ }^{( \pm)} \otimes \hat{\Pi}_{(q-1, m+3)_{n \pm 1}} I_{\overline{1}}^{+} \hat{\Pi}_{(q, m)_{n}} \tilde{\Theta}^{\overline{1}}\right)+2 \sqrt{3} \sum_{ \pm} \varsigma_{2}\left(\phi_{(q, m)_{n}}^{2}( \pm) \dagger \otimes \hat{\Pi}_{(q, m)_{n}} I_{2}^{-} \hat{\Pi}_{(q-1, m-3)_{n \pm 1}} \tilde{\Theta}^{2}+\phi_{(q, m)_{n}}^{2}{ }^{( \pm)}\right. \\
& \left.\otimes \hat{\Pi}_{(q-1, m-3)_{n \pm 1}} I_{2}^{+} \hat{\Pi}_{(q, m)_{n}} \tilde{\Theta}^{\overline{2}}\right)+2 \sqrt{3} \varsigma_{3}\left(\phi_{(q, m)_{n}}^{3} \otimes \hat{\Pi}_{(q, m)_{n}} I_{3}^{-} \hat{\Pi}_{(q+2, m)_{n}} \tilde{\Theta}^{3}+\phi_{(q, m)_{n}}^{3} \otimes \hat{\Pi}_{(q+2, m)_{n}} I_{\overline{3}}^{+} \hat{\Pi}_{(q, m)_{n}} \tilde{\Theta}^{\overline{3})}\right] .
\end{aligned}
$$

The corresponding fields at the Kähler locus of the moduli space can be obtained from (3.9) by setting $\varsigma_{1}=\varsigma_{2}=\frac{1}{\sqrt{2} R}$, $\varsigma_{3}=\frac{1}{2 R}$, and interchanging the generators $I_{3}^{-} \leftrightarrow I_{3}^{+}$and $\tilde{\Theta}^{3} \leftrightarrow \tilde{\Theta}^{\overline{3}}$ (see Appendix A).

The explicit expressions for the matrix elements of the curvature two-form

$$
\mathcal{F}=\mathrm{d} \mathcal{A}+\mathcal{A} \wedge \mathcal{A}
$$

of the connection (3.9) are somewhat complicated; they are listed in Appendix B. Here we content ourselves with displaying the example corresponding to the antifundamental $3 \times 3$ representation $\hat{V}^{0,1}$ of $\mathrm{SU}(3)$; the underlying quiver $Q^{0,1}$ is

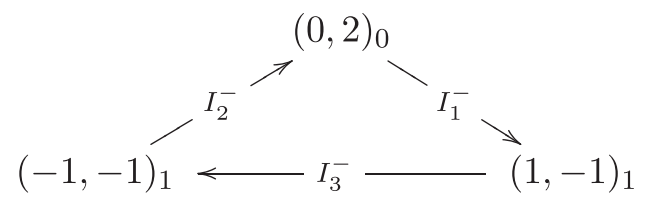

Using the generators (A4) we obtain an SU(3)-invariant gauge connection $\mathcal{A}$ as

$$
\mathcal{A}=\left(\begin{array}{ccc}
A^{1} \otimes 1+\mathbf{1}_{N_{1}} \otimes 2 b & \varsigma_{2} \phi_{2}^{\dagger} \otimes \tilde{\Theta}^{2} & -\varsigma_{1} \phi_{1} \otimes \tilde{\Theta}^{\overline{1}} \\
-\varsigma_{2} \phi_{2} \otimes \tilde{\Theta}^{\overline{2}} & A^{2} \otimes 1+\mathbf{1}_{N_{2}} \otimes\left(\hat{a}_{+}-b\right) & \varsigma_{3} \phi_{3}^{\dagger} \otimes \tilde{\Theta}^{3} \\
\varsigma_{1} \phi_{1}^{\dagger} \otimes \tilde{\Theta}^{1} & -\varsigma_{3} \phi_{3} \otimes \tilde{\Theta}^{\overline{3}} & A^{3} \otimes 1-\mathbf{1}_{N_{3}} \otimes\left(\hat{a}_{+}+b\right)
\end{array}\right),
$$

where $A^{1}, A^{2}$ and $A^{3}$ are $\mathrm{u}\left(N_{1}\right)$-, $\mathrm{u}\left(N_{2}\right)$ - and $\mathrm{u}\left(N_{3}\right)$-valued gauge potentials on Hermitian vector bundles $E_{1}, E_{2}$ and $E_{3}$ over $M$ with ranks $N_{1}, N_{2}$ and $N_{3}$, respectively, such that

$$
N_{1}+N_{2}+N_{3}=N=\operatorname{rank}\left(\mathcal{E}^{0,1}\right),
$$

while $\mathbf{1}_{N_{\alpha}}$ denotes the $N_{\alpha} \times N_{\alpha}$ identity matrix. The bundle morphisms $\phi_{1} \in \operatorname{Hom}\left(E_{3}, E_{1}\right), \phi_{2} \in \operatorname{Hom}\left(E_{1}, E_{2}\right)$ and $\phi_{3} \in$ $\operatorname{Hom}\left(E_{2}, E_{3}\right)$ are bifundamental scalar fields on $M$. The bundles $E_{\alpha}$ are sub-bundles of the quiver bundle

$$
E^{0,1}=E_{1} \otimes \mathbb{C} \oplus E_{2} \otimes \mathbb{C} \oplus E_{3} \otimes \mathbb{C}
$$

over $M$, where the factors $\mathbb{C}$ denote trivial $T$-equivariant line bundles over $M$ arising from the decomposition of the representation $\hat{V}^{0,1} \cong \mathbb{C}^{3}$ into irreducible representations of $T=\mathrm{U}(1) \times \mathrm{U}(1)$. For the curvature $\mathcal{F}=\mathrm{d} \mathcal{A}+\mathcal{A} \wedge \mathcal{A}=$ $\left(\mathcal{F}^{\alpha \beta}\right)$ of the invariant connection (3.11) we obtain

$$
\begin{aligned}
& \mathcal{F}^{11}=F^{1}-\varsigma_{1}^{2}\left(\mathbf{1}_{N_{1}}-\phi_{1} \phi_{1}^{\dagger}\right) \tilde{\Theta}^{1} \wedge \tilde{\Theta}^{\overline{1}}+\varsigma_{2}^{2}\left(\mathbf{1}_{N_{1}}-\phi_{2}^{\dagger} \phi_{2}\right) \tilde{\Theta}^{2} \wedge \tilde{\Theta}^{\overline{2}}, \\
& \mathcal{F}^{22}=F^{2}-\varsigma_{2}^{2}\left(\mathbf{1}_{N_{2}}-\phi_{2} \phi_{2}^{\dagger}\right) \tilde{\Theta}^{2} \wedge \tilde{\Theta}^{\overline{2}}+\varsigma_{3}^{2}\left(\mathbf{1}_{N_{2}}-\phi_{3}^{\dagger} \phi_{3}\right) \tilde{\Theta}^{3} \wedge \tilde{\Theta}^{\overline{3}}, \\
& \mathcal{F}^{33}=F^{3}-\varsigma_{3}^{2}\left(\mathbf{1}_{N_{3}}-\phi_{3} \phi_{3}^{\dagger}\right) \tilde{\Theta}^{3} \wedge \tilde{\Theta}^{\overline{3}}+\varsigma_{1}^{2}\left(\mathbf{1}_{N_{3}}-\phi_{1}^{\dagger} \phi_{1}\right) \tilde{\Theta}^{1} \wedge \tilde{\Theta}^{\overline{1}}, \\
& \mathcal{F}^{13}=-\varsigma_{1}\left(\mathrm{~d} \phi_{1}+A^{1} \phi_{1}-\phi_{1} A^{3}\right) \wedge \tilde{\Theta}^{\overline{1}}-\varsigma_{2} \varsigma_{3}\left(\phi_{1}-\phi_{2}^{\dagger} \phi_{3}^{\dagger}\right) \tilde{\Theta}^{2} \wedge \tilde{\Theta}^{3}, \\
& \mathcal{F}^{21}=-\varsigma_{2}\left(\mathrm{~d} \phi_{2}+A^{2} \phi_{2}-\phi_{2} A^{1}\right) \wedge \tilde{\Theta}^{\overline{2}}-\varsigma_{1} \varsigma_{3}\left(\phi_{2}-\phi_{3}^{\dagger} \phi_{1}^{\dagger}\right) \tilde{\Theta}^{3} \wedge \tilde{\Theta}^{1}, \\
& \mathcal{F}^{32}=-\varsigma_{3}\left(\mathrm{~d} \phi_{3}+A^{3} \phi_{3}-\phi_{3} A^{2}\right) \wedge \tilde{\Theta}^{\overline{3}}-\varsigma_{1} \varsigma_{2}\left(\phi_{3}-\phi_{1}^{\dagger} \phi_{2}^{\dagger}\right) \tilde{\Theta}^{1} \wedge \tilde{\Theta}^{2},
\end{aligned}
$$


plus their Hermitian conjugates $\mathcal{F}^{\beta \alpha}=-\left(\mathcal{F}^{\alpha \beta}\right)^{\dagger}$ for $\alpha \neq$ $\beta$. In (3.14) the superscripts $\alpha, \beta$ label $N_{\alpha} \times N_{\beta}$ blocks in $\mathcal{F}$, and we have suppressed tensor products in order to simplify notation. Here $F^{\alpha}=\mathrm{d} A^{\alpha}+A^{\alpha} \wedge A^{\alpha}$ is the curvature of the connection $A^{\alpha}$ on the complex vector bundle $E_{\alpha} \rightarrow M$.

\section{B. Invariant spinor fields}

We assume henceforth that $M$ is a spin manifold of even dimension $d$ for simplicity, together with a fixed metric. The equivariant dimensional reduction of Dirac spinors on $\mathcal{M}=M \times \mathbb{F}_{3}$ is defined with respect to (twisted) symmetric fermions on $M$. They act as intertwining operators connecting induced representations of the maximal torus $T=\mathrm{U}(1) \times \mathrm{U}(1)$ in the $\mathrm{U}(N)$ gauge group, and also in the twisted spinor module $\Delta$ over $M$, which admits the isotopical decomposition

$$
\begin{aligned}
\Delta^{k, l} & =\bigoplus_{(q, m)_{n} \in W^{k, l}} \Delta_{q, m} \otimes V_{(q, m)_{n}} \text { with } \\
\Delta_{q, m} & =\operatorname{Hom}_{T}\left(V_{(q, m)_{n}}, \Delta^{k, l}\right)
\end{aligned}
$$

obtained by restricting $\Delta$ to induced representations $\left.\hat{V}^{k, l}\right|_{T}$ of $T \subset \operatorname{Spin}(d)$. Using Frobenius reciprocity, the multiplicity spaces may be identified as

$\Delta_{q, m}=\operatorname{Hom}_{G}\left(\Delta^{k, l}, \mathrm{~L}^{2}\left(\mathbb{F}_{3} ;\left(\mathcal{L}_{(1)}\right)^{\otimes q} \otimes\left(\mathcal{L}_{(2)}\right)^{\otimes m}\right)\right)$,

and hence the isotopical decomposition (3.15) can be realized explicitly by constructing symmetric fermions on $M$ as $\mathrm{SU}(3)$-invariant spinors on $M \times \mathbb{F}_{3}$. They are associated with the spinor harmonics of the twisted Dirac operator on $\mathbb{F}_{3}$. Dirac zero modes can be used to construct an $\mathrm{SU}(3)$-equivariant spinor field $\Psi$ on $\mathcal{M}=M \times \mathbb{F}_{3}$, which decomposes into invariant subspaces. The decomposition is simplest when the index reflects the actual number of zero modes, i.e. $\nu_{q, m}=\operatorname{dim} \operatorname{ker}\left(\not \not_{q, m}^{+}\right)$for $(q, m)_{n} \in W_{+}^{k, l}$ and $\nu_{q, m}=-\operatorname{dim} \operatorname{ker}\left(\not \not_{q, m}^{-}\right)$for $(q, m)_{n} \in$ $W_{-}^{k, l}$. Then the decomposition is $\Psi=\Psi^{+} \oplus \Psi^{-}$with

$$
\begin{aligned}
\Psi^{+} & :=\bigoplus_{(q, m)_{n} \in W_{+}^{k, l}} \Psi_{(q, m)_{n}}^{+} \text {with } \\
\Psi_{(q, m)_{n}}^{+} & =\sum_{\ell=1}^{\nu_{q, m}} \psi_{(q, m)_{n} ; \ell} \otimes \chi_{q, m ; \ell}^{+}, \\
\Psi^{-} & :=\bigoplus_{(q, m)_{n} \in W_{-}^{k, l}} \Psi_{(q, m)_{n}}^{-} \text {with } \\
\Psi_{(q, m)_{n}}^{-} & =\sum_{\ell=1}^{\left|\nu_{q, m}\right|} \breve{\psi}_{(q, m)_{n} ; \ell} \otimes \chi_{q, m ; \ell}^{-},
\end{aligned}
$$

where $\psi_{(q, m)_{n} ; \ell}$ and $\breve{\psi}_{(q, m)_{n} ; \ell}$ are Dirac spinors on $M$, and $\chi_{q, m ; \ell}^{ \pm}$the positive/negative chirality zero modes on $\mathbb{F}_{3}$.

More generally let $n_{q, m}^{ \pm}=\operatorname{dim} \operatorname{ker}\left(\not D_{q, m}^{ \pm}\right)$, with $\nu_{q, m}=$ $n_{q, m}^{+}-n_{q, m}^{-}$, and then the decomposition is $\Psi=$ $\Psi^{+} \oplus \Psi^{0} \oplus \Psi^{-}$with

$$
\begin{aligned}
\Psi^{+} & :=\bigoplus_{(q, m)_{n} \in W_{+}^{k, l}} \Psi_{(q, m)_{n}}^{+} \text {with } \\
\Psi_{(q, m)_{n}}^{+} & =\sum_{\ell=1}^{n_{q, m}^{+}} \psi_{(q, m)_{n} ; \ell} \otimes \chi_{q, m ; \ell}^{+}+\sum_{\ell^{\prime}=1}^{n_{q, m}^{-}} \eta_{(q, m)_{n} ; \ell^{\prime}} \otimes \chi_{q, m ; \ell^{\prime}}^{-}, \\
\Psi^{0} & :=\bigoplus_{(q, m)_{n} \in W_{0}^{k, l}} \Psi_{(q, m)_{n}}^{0} \text { with } \\
\Psi_{(q, m)_{n}}^{0}= & \sum_{\ell=1}^{n_{q, m}^{+}} \psi_{(q, m)_{n} ; \ell} \otimes \chi_{q, m ; \ell}^{+}+\sum_{\ell^{\prime}=1}^{n_{q, m}^{-}} \eta_{(q, m)_{n} ; \ell^{\prime}} \otimes \chi_{q, m ; \ell^{\prime}}^{-}, \\
\Psi^{-} & :=\bigoplus_{(q, m)_{n} \in W_{-}^{k, l}} \Psi_{(q, m)_{n}}^{-} \text {with } \\
\Psi_{(q, m)_{n}}^{-} & =\sum_{\ell=1}^{n_{q, m}^{-}} \eta_{(q, m)_{n} ; \ell} \otimes \chi_{q, m ; \ell}^{-}+\sum_{\ell^{\prime}=1}^{n_{q, m}^{+}} \psi_{(q, m)_{n} ; \ell^{\prime}} \otimes \chi_{q, m ; \ell^{\prime}}^{+} .
\end{aligned}
$$

For convenience we shall give formulas below only for the case (3.17); the extension to the more general case (3.18) is straightforward but notationally more cumbersome [an exception to this will be the specific analysis of the adjoint $(k, l)=(1,1)$, where $(q, m)=(0,0)$ has index $\nu_{0,0}=0$ but $n_{0,0}^{+}=n_{0,0}^{-}=1$ and $\Psi^{0}$ is nonzero].

To describe the Dirac operator acting on the invariant spinor fields (3.17), we need to choose bases for the pertinent Clifford algebras. For the Clifford algebra of $M$ we first choose $2^{d / 2} \times 2^{d / 2}$ anti-Hermitian gamma matrices $\gamma^{\mu}$ obeying the anticommutation relations

$$
\left\{\gamma^{\mu}, \gamma^{\nu}\right\}=2 \eta^{\mu \nu} \mathbf{1}_{2^{d / 2}}
$$

with $\mu, \nu=1, \ldots, d$ (real) orthonormal indices. The basis of gamma matrices on $\mathbb{F}_{3}$ obeys the Clifford relations (2.33). Then a suitable basis for the Clifford algebra on $M \times \mathbb{F}_{3}$ is given by the gamma matrices $\left\{\tilde{\Gamma}^{A}\right\}=$ $\left\{\tilde{\Gamma}^{\mu}, \tilde{\Gamma}^{\alpha}, \tilde{\Gamma}^{\bar{\alpha}}\right\}$ for $\mu=1, \ldots, d, \alpha=1,2,3$ defined as

$$
\tilde{\Gamma}^{\mu}=\gamma^{\mu} \otimes \mathbf{1}_{8}, \quad \tilde{\Gamma}^{\alpha}=\gamma \otimes \tilde{\gamma}^{\alpha} \quad \text { and } \quad \tilde{\Gamma}^{\bar{\alpha}}=\gamma \otimes \tilde{\gamma}^{\bar{\alpha}},
$$

where $\gamma=(-1)^{d(d-1) / 2} \gamma^{1} \cdots \gamma^{d}$ is the chirality operator on $M$ that anticommutes with all generators $\gamma^{\mu}$, and satisfies $\gamma=\gamma^{\dagger}$ and $(\gamma)^{2}=\mathbf{1}_{2^{d / 2}}$.

The $E^{k, l}$-twisted Dirac operator on $M$ is given by

$$
\not D=\sum_{(q, m)_{n} \in W^{k, l}}\left(\not \not_{M}+\not \not^{(q, m)_{n}}\right) \otimes \Pi_{(q, m)_{n}},
$$

where $\not \not_{M}$ is the Dirac operator on $M$ involving only the Levi-Cività spin connection. Together with the Dirac operators (2.35) on $\mathbb{F}_{3}$ and the map (2.34), the $\mathcal{E}^{k, l}$-twisted Dirac operator $\mathcal{D}$ on $\mathcal{M}=M \times \mathbb{F}_{3}$, corresponding to the equivariant gauge potential $\mathcal{A}$ in (3.9) and acting on the spinor fields (3.17), can be written as 


$$
\mathcal{D}=\not D \otimes \mathbf{1}_{8}+\gamma \otimes \not_{\mathbb{F}_{3}}+\tilde{y},
$$

where

$$
\not \mathbb{E}_{\mathbb{F}_{3}}=\sum_{(q, m)_{n} \in W^{k, l}} \not D_{q, m} \otimes \hat{\Pi}_{(q, m)_{n}}
$$

is the $\mathrm{SU}(3)$-equivariant twisted Dirac operator acting on eight-component spinor fields on $\mathbb{F}_{3}$, and

$$
\begin{aligned}
\widetilde{y}= & 2 \sqrt{3} \sum_{(q, m)_{n} \in W^{k,}}\left[\sum_{ \pm} \varsigma_{1}\left(\phi_{(q, m)_{n}}^{1}{ }^{( \pm) \dagger} \gamma \otimes \hat{\Pi}_{(q, m)_{n}} I_{1}^{-} \hat{\Pi}_{(q-1, m+3)_{n \pm 1}} \tilde{\gamma}^{1}+\phi_{(q, m)_{n}}^{1}{ }^{( \pm)} \gamma \otimes \hat{\Pi}_{(q-1, m+3)_{n \pm 1}} I_{\overline{1}}^{+} \hat{\Pi}_{(q, m)_{n}} \tilde{\gamma}^{\overline{1}}\right)\right. \\
& +\sum_{ \pm} \varsigma_{2}\left(\phi_{(q, m)_{n}}^{2}{ }^{( \pm) \dagger} \dagger \otimes \hat{\Pi}_{(q, m)_{n}} I_{2}^{-} \hat{\Pi}_{(q-1, m-3)_{n \pm 1}} \tilde{\gamma}^{2}+\phi_{(q, m)_{n}}^{2}{ }^{( \pm)} \gamma \otimes \hat{\Pi}_{(q-1, m-3)_{n \pm 1}} I_{\overline{2}}^{+} \hat{\Pi}_{(q, m)_{n}} \tilde{\gamma}^{\overline{2}}\right) \\
& \left.+\varsigma_{3}\left(\phi_{(q, m)_{n}}^{3}{ }^{\dagger} \gamma \otimes \hat{\Pi}_{(q, m)_{n}} I_{3}^{-} \hat{\Pi}_{(q+2, m)_{n}} \tilde{\gamma}^{3}+\phi_{(q, m)_{n}}^{3} \gamma \otimes \hat{\Pi}_{(q+2, m)_{n}} I_{\overline{3}}^{+} \hat{\Pi}_{(q, m)_{n}} \tilde{\gamma}^{\overline{3}}\right)\right] .
\end{aligned}
$$

This construction also demonstrates that there is a bijective correspondence between states (2.45) of the eightdimensional spinor module $\Delta_{\mathbb{F}_{3}}$ and the weight space of the adjoint representation of SU(3): From (3.24) it follows that the action of the $\gamma$ matrices $\tilde{\gamma}^{\alpha}$ on the quiver lattice $W^{k, l}$ is given by

$$
\begin{aligned}
& \tilde{\gamma}^{1}:(q-1, m+3)_{n \pm 1} \rightarrow(q, m)_{n}, \\
& \tilde{\gamma}^{2}:(q-1, m-3)_{n \pm 1} \rightarrow(q, m)_{n}, \\
& \tilde{\gamma}^{3}:(q+2, m)_{n} \rightarrow(q, m)_{n},
\end{aligned}
$$

and hence

$$
\begin{aligned}
& \tilde{\gamma}^{23}:(q, m)_{n} \rightarrow(q-1, m+3)_{n \pm 1}, \\
& \tilde{\gamma}^{31}:(q, m)_{n} \rightarrow(q-1, m-3)_{n \pm 1}, \\
& \tilde{\gamma}^{12}:(q, m)_{n} \rightarrow(q+2, m)_{n},
\end{aligned}
$$

while $\tilde{\gamma}^{123}$ and the identity $\mathbf{1}_{8}$ both leave a state with weight $(q, m)_{n}$ fixed. These transformation properties reflect the $\mathrm{U}(1) \times \mathrm{U}(1)$ charges of the Clifford algebra generators, which fill out the weight lattice $W^{1,1}$ of the adjoint representation $\left.\hat{V}^{1,1}\right|_{T}$. In particular, there is a natural correspondence $\tilde{\gamma}^{\alpha} \leftrightarrow 2 \sqrt{3} I_{\alpha}^{-}$and $\tilde{\gamma}^{\bar{\alpha}} \leftrightarrow 2 \sqrt{3} I_{\bar{\alpha}}^{+}$(this correspondence is, of course, not an algebra isomorphism). The significance of the adjoint representation here will become clear in Sec. V B; recall from Sec. IID that it is the smallest irreducible $\mathrm{SU}(3)$ module that accommodates harmonic spinors on $\mathbb{F}_{3}$ with a nonzero index.

\section{VACUUM STRUCTURE OF INDUCED YANG-MILLS-HIGGS THEORY}

In this section we study the quiver gauge theory on $M$ obtained by the dimensional reduction of Yang-Mills theory on $\mathcal{M}=M \times \mathbb{F}_{3}$ over $\mathbb{F}_{3}$. The reductions of Hermitian Yang-Mills equations for such systems, describing the consistent stable solutions of the quiver gauge theory, are analyzed in detail in [12] and related to quiver vortex equations in certain instances; in the present paper we are interested in the detailed vacuum structure of these solutions.

\section{A. Reduction of the Yang-Mills action}

We will begin with the case of the antifundamental representation $\hat{V}^{0,1}$. The dimensionally reduced action $S_{M}^{\mathrm{YM}}$ on $M$ is obtained by integrating the Yang-Mills action $S_{\mathcal{M}}^{\mathrm{YM}}$ on $\mathcal{M}$ over the coset space $\mathbb{F}_{3}$. The $(d+6)$ dimensional Lagrangian on $\mathcal{M}$ is the $(d+6)$-form

$$
\begin{aligned}
\mathcal{L}_{\mathcal{M}}^{\mathrm{YM}} & =\frac{1}{4 \tilde{g}^{2}} \operatorname{Tr}_{N}\left(\mathcal{F} \wedge \tilde{\star} \mathcal{F}^{\dagger}\right) \\
& =-\frac{1}{4 \tilde{g}^{2}} \operatorname{Tr}_{N}\left(\bigoplus_{\alpha, \beta=1}^{3} \mathcal{F}^{\alpha \beta} \wedge \tilde{\star} \mathcal{F}^{\beta \alpha}\right),
\end{aligned}
$$

where $\operatorname{Tr}_{N}$ is the trace in the fundamental representation of $\mathrm{U}(N), \tilde{\star}$ is the Hodge duality operator on $\mathcal{M}$ compatible with the orthonormal frame $\tilde{\Theta}^{\alpha}$, and a dimensionful Yang-Mills coupling constant $\tilde{g} \in \mathbb{R}$ has been included. Using (3.14) this gives

$$
\begin{aligned}
\mathcal{L}_{\mathcal{M}}^{\mathrm{YM}}= & \frac{1}{4 \tilde{g}^{2}} \sum_{\alpha=1}^{3} \operatorname{Tr}_{N_{\alpha}}\left(-F^{\alpha} \wedge \tilde{\star} F^{\alpha}+s_{\alpha}^{2}\left(D \phi_{\alpha}\right)\right. \\
& \left.\wedge \tilde{\star}\left(D \phi_{\alpha}\right)^{\dagger}+\mathrm{s}_{\alpha+1}^{2}\left(D \phi_{\alpha+1}\right)^{\dagger} \wedge \tilde{\star}\left(D \phi_{\alpha+1}\right)\right) \\
& +\tilde{V}^{\mathrm{YM}}(\phi) \wedge \tilde{\star} 1,
\end{aligned}
$$

where throughout we read the indices $\alpha=1,2,3$ modulo 3 and

$$
D \phi_{\alpha}:=\mathrm{d} \phi_{\alpha}+A^{\alpha} \phi_{\alpha}-\phi_{\alpha} A^{\alpha+2}
$$

are bifundamental covariant derivatives of the Higgs fields $\phi_{\alpha} \in \operatorname{Hom}\left(E_{\alpha+2}, E_{\alpha}\right)$. The Higgs potential is given by

$$
\begin{aligned}
\tilde{V}^{\mathrm{YM}}(\phi)= & \frac{1}{4 \tilde{g}^{2}} \sum_{\alpha=1}^{3} \operatorname{Tr}_{N_{\alpha}}\left(\boldsymbol{s}_{\alpha}^{4}\left(\mathbf{1}_{N_{\alpha}}-\phi_{\alpha} \phi_{\alpha}^{\dagger}\right)^{2}\right. \\
& +\varsigma_{\alpha+1}^{4}\left(\mathbf{1}_{N_{\alpha}}-\phi_{\alpha+1}^{\dagger} \phi_{\alpha+1}\right)^{2} \\
& \left.+\varsigma_{\alpha+2}^{2} \varsigma_{\alpha}^{2}\left|\phi_{\alpha+1}-\phi_{\alpha+2}^{\dagger} \phi_{\alpha}^{\dagger}\right|^{2}\right)
\end{aligned}
$$

where we use the matrix notation $|\phi|^{2}:=\frac{1}{2}\left(\phi^{\dagger} \phi+\phi \phi^{\dagger}\right)$.

The action $S_{\mathcal{M}}^{\mathrm{YM}}=\int_{\mathcal{M}} \mathcal{L}_{\mathcal{M}}^{\mathrm{YM}}$ is made dimensionless here by taking the coupling $\tilde{g}$ to have mass dimension $-\frac{d}{2}-1$ 
and the metric moduli $\varsigma_{\alpha}$ mass dimension 1 , so that $\phi_{\alpha}$ are then dimensionless while $A^{\alpha}$ have mass dimension 1 . This gives the dimensionally reduced Lagrangian $d$-form

$$
\begin{aligned}
\mathcal{L}_{M}^{\mathrm{YM}}:= & \int_{\mathbb{F}_{3}} \mathcal{L}_{\mathcal{M}}^{\mathrm{YM}} \\
= & \frac{1}{4 g^{2}} \sum_{\alpha=1}^{3} \operatorname{Tr}_{N_{\alpha}}\left(-F^{\alpha} \wedge \star F^{\alpha}+\varsigma_{\alpha}^{2}\left(D \phi_{\alpha}\right)\right. \\
& \wedge \star\left(D \phi_{\alpha}\right)^{\dagger}+\varsigma_{\alpha+1}^{2}\left(D \phi_{\alpha+1}\right)^{\dagger} \\
& \left.\wedge \star\left(D \phi_{\alpha+1}\right)\right)+V^{\mathrm{YM}}(\phi) \wedge \star 1
\end{aligned}
$$

with

$$
\begin{aligned}
V^{\mathrm{YM}}(\phi)= & \frac{1}{4 g^{2}} \sum_{\alpha=1}^{3} \operatorname{Tr}_{N_{\alpha}}\left(\boldsymbol{\varsigma}_{\alpha}^{4}\left(\mathbf{1}_{N_{\alpha}}-\phi_{\alpha} \phi_{\alpha}^{\dagger}\right)^{2}\right. \\
& +\varsigma_{\alpha+1}^{4}\left(\mathbf{1}_{N_{\alpha}}-\phi_{\alpha+1}^{\dagger} \phi_{\alpha+1}\right)^{2} \\
& \left.+\varsigma_{\alpha+2}^{2} \varsigma_{\alpha}^{2}\left|\phi_{\alpha+1}-\phi_{\alpha+2}^{\dagger} \phi_{\alpha}^{\dagger}\right|^{2}\right),
\end{aligned}
$$

where $g^{-2}:=\operatorname{Vol}\left(\mathbb{F}_{3}\right) \tilde{g}^{-2}$ with $\operatorname{Vol}\left(\mathbb{F}_{3}\right)=\int_{\mathbb{F}_{3}} \tilde{\omega}^{\wedge 3} / 3$ ! the volume of $\mathbb{F}_{3}$, so that the coupling constant $g$ has dimension $2-\frac{d}{2}$, and $\star$ is the $d$-dimensional Hodge duality operator on $M$.

\section{B. Reduction of the Chern-Simons torsion coupling}

As a subsector of heterotic supergravity, the Yang-Mills gauge theory also contains a coupling to a torsional 3-form flux $\mathcal{H}$ on $\mathcal{M}$. There is a natural candidate for a 3 -form on $\mathbb{F}_{3}$, which extends trivially to a 3 -from on $\mathcal{M}$,

$$
\mathcal{H}=\tilde{\star} \mathrm{d} \Sigma=\star_{\mathbb{F}_{3}} \mathrm{~d} \tilde{\omega} \quad \text { with } \quad \Sigma:=\tilde{\omega} \wedge \star 1,
$$

which satisfies $\mathrm{d} \tilde{\star} \mathcal{H}=0$, with $\star_{\mathbb{F}_{3}}$ the Hodge duality operator on $\mathbb{F}_{3}$ with respect to the orthonormal frame $\tilde{\Theta}^{\alpha}$. The torsion coupling is then given via the Chern-Simons 3 -form of the gauge potential $\mathcal{A}$ through the Lagrangian $(d+6)$-form

$\mathcal{L}_{\mathcal{M}}^{\mathrm{CS}}=\tilde{\kappa} \operatorname{Tr}_{N}\left(\mathcal{F} \wedge \mathcal{A}-\frac{1}{3} \mathcal{A} \wedge \mathcal{A} \wedge \mathcal{A}\right) \wedge \tilde{\star} \mathcal{H}$,

where the coupling constant $\tilde{\kappa} \in \mathbb{R}$ has mass dimension $d$. We may compute the reduction of (4.8) over the coset space $\mathbb{F}_{3}$ by first observing that integration by parts of this form yields the equivalent form $\mathcal{L}_{\mathcal{M}}^{\mathrm{CS}}=$ $\tilde{\kappa} \operatorname{Tr}_{N}(\mathcal{F} \wedge \mathcal{F}) \wedge \Sigma$ when $M$ is closed, and then substituting (2.32) and (3.14). After integration over $\mathbb{F}_{3}$, this becomes solely an additional contribution to the Higgs potential (4.6) given by

$$
\begin{aligned}
V^{\mathrm{CS}}(\phi)= & \frac{\kappa}{2} \sum_{\alpha=1}^{3} \operatorname{Tr}_{N_{\alpha}}\left(2 \varsigma_{\alpha}^{2} \varsigma_{\alpha+1}^{2}\left(\mathbf{1}_{N_{\alpha}}-\phi_{\alpha} \phi_{\alpha}^{\dagger}\right)\right. \\
& \times\left(\mathbf{1}_{N_{\alpha}}-\phi_{\alpha+1}^{\dagger} \phi_{\alpha+1}\right) \\
& \left.-\varsigma_{\alpha+2}^{2} \varsigma_{\alpha}^{2}\left|\phi_{\alpha+1}-\phi_{\alpha+2}^{\dagger} \phi_{\alpha}^{\dagger}\right|^{2}\right),
\end{aligned}
$$

where the coupling constant $\kappa:=\operatorname{Vol}\left(\mathbb{F}_{3}\right) \tilde{\kappa}$ has dimension $d-6$. The effects of this torsion coupling disappear in the classical field theory at both the Kähler locus (where $\mathrm{d} \tilde{\omega}=0$ ) and the nearly Kähler locus [where $\mathrm{d} \tilde{\omega}$ is a sum of $(3,0)$ - and $(0,3)$-forms] of the moduli space.

However, in heterotic string backgrounds not all of the intrinsic torsion appears in the Neveu-Schwarz 3 -form $\mathcal{H}$; for example, the nontrivial relation between the torsion classes $W_{4}$ and $W_{5}$ determines the dilaton, which we have ignored in light of our nonsupersymmetric analysis. Moreover, the nontrivial Bianchi identity satisfied by the 3 -form $\mathcal{H}$ leads to very stringent consistency conditions; see [12] for an analysis of the reductions involving more general torsion fluxes and [8] for a description of the general torsion flux constraints.

\section{Vacuum states}

We will now study the dynamical symmetry breaking patterns in the Yang-Mills-Higgs theory derived above. For this, it is convenient to rescale

$\boldsymbol{\varsigma}_{\alpha}^{\prime}=\frac{\boldsymbol{s}_{\alpha}}{2 g}, \quad \phi_{\alpha}^{\prime}=\boldsymbol{\varsigma}_{\alpha}^{\prime} \phi_{\alpha} \quad$ and $\quad A^{\prime \alpha}=\frac{1}{g} A^{\alpha}$,

so that the scalar field $\phi_{\alpha}^{\prime}$ and the gauge potential $A^{\prime \alpha}$ both have the conventional physical mass dimension $\frac{d}{2}-1$. Then (4.5) becomes

$$
\begin{aligned}
\mathcal{L}_{M}^{\mathrm{YM}}= & \sum_{\alpha=1}^{3} \operatorname{Tr}_{N_{\alpha}}\left(-\frac{1}{4} F^{\prime \alpha} \wedge \star F^{\prime \alpha}+\left(D^{\prime} \phi_{\alpha}^{\prime}\right)\right. \\
& \left.\wedge \star\left(D^{\prime} \phi_{\alpha}^{\prime}\right)^{\dagger}+\left(D^{\prime} \phi_{\alpha+1}^{\prime}\right)^{\dagger} \wedge \star\left(D^{\prime} \phi_{\alpha+1}^{\prime}\right)\right) \\
& +V^{\mathrm{YM}}\left(\phi^{\prime}\right) \wedge \star 1,
\end{aligned}
$$

with

$$
\begin{aligned}
V^{\mathrm{YM}}\left(\phi^{\prime}\right)= & 4 g^{2} \sum_{\alpha=1}^{3} \operatorname{Tr}_{N_{\alpha}}\left(\left(\boldsymbol{s}_{\alpha}^{\prime}{ }^{2} \mathbf{1}_{N_{\alpha}}-\phi_{\alpha}^{\prime} \boldsymbol{\phi}_{\alpha}^{\prime \dagger}\right)^{2}\right. \\
& +\left(\boldsymbol{s}_{\alpha+1}^{\prime} \mathbf{1}_{N_{\alpha}}-\phi_{\alpha+1}^{\prime}{ }^{\dagger} \phi_{\alpha+1}^{\prime}\right)^{2} \\
& \left.+\left|\frac{\mathbf{s}_{\alpha+2}^{\prime} \mathbf{s}_{\alpha}^{\prime}}{\mathbf{s}_{\alpha+1}^{\prime}} \phi_{\alpha+1}^{\prime}-\phi_{\alpha+2}^{\prime}{ }^{\dagger} \phi_{\alpha}^{\prime}\right|^{2}\right)
\end{aligned}
$$

Every term in (4.12) is non-negative; hence the absolute minimum is achieved by the field configurations satisfying

$$
\begin{aligned}
\phi_{\alpha}^{\prime} \phi_{\alpha}^{\prime}{ }^{\dagger} & =\boldsymbol{s}_{\alpha}^{\prime}{ }^{2} \mathbf{1}_{N_{\alpha}}, \\
\phi_{\alpha+1}^{\prime}{ }^{\dagger} \phi_{\alpha+1}^{\prime} & =\boldsymbol{\varsigma}_{\alpha+1}^{\prime}{ }^{2} \mathbf{1}_{N_{\alpha}} \text { and } \\
\boldsymbol{s}_{\alpha+1}^{\prime} \boldsymbol{s}_{\alpha+2}^{\prime} \phi_{\alpha}^{\prime} & =\boldsymbol{s}_{\alpha}^{\prime} \phi_{\alpha+1}^{\prime}{ }^{\dagger} \phi_{\alpha+2}^{\prime}{ }^{\dagger}
\end{aligned}
$$

for $\alpha=1,2,3$.

Rather than exhibiting the full complication of the most general case, which rapidly becomes notationally very cumbersome, for illustrative purposes we shall only 
consider the case $N \equiv 0 \bmod 3$ with $N_{\alpha}=p:=\frac{N}{3}$ for $\alpha=$ $1,2,3$. The gauge group is then $\mathrm{U}(p)^{3}$, with $3 p^{2}$ gauge bosons. The vacuum solution that minimizes the Higgs potential and kinetic energies is then given by

$$
\phi_{\alpha}^{\prime}=\mathrm{s}_{\alpha}^{\prime} V_{\alpha},
$$

with $V_{\alpha} \in \mathrm{U}(p)$ constant unitary matrices satisfying the condition

$$
V_{3} V_{2} V_{1}=\mathbf{1}_{p}
$$

As in [14], the Higgs vacuum thus provides a representation of the relations of the double quiver $\bar{Q}^{0,1}$ associated with the antifundamental representation $\hat{V}^{0,1}$ of $\mathrm{SU}(3)$, obtained by adding an arrow in the opposite direction to each arrow of the quiver $Q^{0,1}$, which ensures that there are no nontrivial oriented cycles in $\bar{Q}^{0,1}$ (and hence that finitedimensional quiver representations are possible); it defines a flat connection of the $\mathrm{U}(p)$ lattice gauge theory on the finite quiver lattice.

We obtain the mass matrix for the gauge bosons by substituting this vacuum solution into the bicovariant derivative terms $D^{\prime} \phi_{\alpha}^{\prime}$ in (4.11) and extracting the part quadratic in the gauge potentials $A^{\prime \alpha}$ from the Higgs field kinetic terms. The mass matrix $\boldsymbol{M}$ is then given by

$$
M^{2}=4 g^{2}\left(\begin{array}{ccc}
\varsigma_{1}^{\prime 2}+\varsigma_{2}^{\prime 2} & -\varsigma_{2}^{\prime 2} & -\varsigma_{1}^{\prime 2} \\
-\varsigma_{2}^{\prime 2} & \varsigma_{2}^{\prime 2}+\varsigma_{3}^{\prime 2} & -\varsigma_{3}^{\prime 2} \\
-\varsigma_{1}^{\prime 2} & -\varsigma_{3}^{\prime 2} & \varsigma_{1}^{\prime 2}+\varsigma_{3}^{\prime 2}
\end{array}\right) .
$$

There is a single zero eigenvalue, corresponding to the massless diagonal combination $\frac{1}{\sqrt{3}}\left(A^{1}+A^{2}+A^{3}\right)$, and two nonzero eigenvalues

$$
\begin{aligned}
M_{ \pm}^{2}= & 4 g^{2}\left(s_{1}^{\prime 2}+\varsigma_{2}^{\prime 2}+\varsigma_{3}^{\prime 2}\right. \\
& \left. \pm \sqrt{\varsigma_{1}^{\prime 4}+s_{2}^{\prime 4}+\varsigma_{3}^{\prime 4}-\left(s_{1}^{\prime 2} s_{2}^{\prime 2}+\varsigma_{2}^{\prime 2} s_{3}^{\prime 2}+\varsigma_{3}^{\prime 2} s_{1}^{\prime 2}\right)}\right) .
\end{aligned}
$$

Thus the gauge group $\mathrm{U}(p)^{3}$ is broken to its diagonal subgroup $\mathrm{U}(p)_{\text {diag }}$, and the remaining $2 p^{2}$ gauge bosons acquire the masses $M_{ \pm}$. In the nearly Kähler and Kähler reductions of the general $\mathrm{SU}(3)$ structure on $\mathbb{F}_{3}$ these masses become

$$
\begin{aligned}
& \text { Nearly Kähler: } M_{+}=M_{-}=\frac{\sqrt{3}}{2 R}, \\
& \text { Kähler: } M_{+}=\frac{\sqrt{6}}{2 R}, \quad M_{-}=\frac{1}{R} .
\end{aligned}
$$

Note that the nearly Kähler locus in the moduli space yields degenerate mass eigenvalues.

At a generic point in the moduli space one should also incorporate the potential (4.9) arising from the torsion coupling in the supergravity equations. The main effect of this addition is that it generally introduces nonpositive terms in the potential and leads to a larger vacuum moduli space of solutions. In particular, at the special coupling value

$$
\kappa=-\frac{1}{2 g^{2}},
$$

the first lines of the potentials (4.6) and (4.9) complete to a perfect square, and the total potential is a sum of nonnegative terms; the vacuum equations (4.13) are then modified to

$$
\begin{aligned}
\phi_{\alpha}^{\prime} \phi_{\alpha}^{\prime}{ }^{\dagger}-\phi_{\alpha+1}^{\prime}{ }^{\dagger} \phi_{\alpha+1}^{\prime} & =\left(\boldsymbol{\varsigma}_{\alpha}^{\prime}{ }^{2}-\boldsymbol{\varsigma}_{\alpha+1}^{\prime}{ }^{2}\right) \mathbf{1}_{N_{\alpha}} \text { and } \\
\boldsymbol{\varsigma}_{\alpha+1}^{\prime} \boldsymbol{\varsigma}_{\alpha+2}^{\prime} \phi_{\alpha}^{\prime} & =\boldsymbol{\varsigma}_{\alpha}^{\prime} \phi_{\alpha+1}^{\prime}{ }^{\dagger} \boldsymbol{\phi}_{\alpha+2}^{\prime}{ }^{\dagger} .
\end{aligned}
$$

These equations represent, respectively, the moment map equations and relations of the double quiver $\bar{Q}^{0,1}$ [12]. Any solution of (4.13) is also a solution of (4.20), but not conversely. The relationship (4.19) between the YangMills and Chern-Simons coupling constants also appears in heterotic supergravity, wherein both couplings are proportional to $g_{s}^{2} \alpha^{\prime}$ with $g_{s}$ the string coupling constant and $\alpha^{\prime}$ the Regge slope.

$N=3$.- To understand the structure of the Higgs potential, we first consider the case $p=1$, which gives rise to an Abelian gauge theory: $\mathrm{U}(1)^{3}$ is broken to $\mathrm{U}(1)_{\text {diag }}$. There are three complex Higgs fields, two gauge bosons acquire a mass and, of the six real scalar fields in the Higgs sector, four are physical. The Higgs vacuum is of the form

$$
\phi_{\alpha}^{\prime}=\varsigma_{\alpha}^{\prime} \mathrm{e}^{\mathrm{i} \zeta_{\alpha}},
$$

where $\zeta_{\alpha}, \alpha=1,2,3$ are three phases constrained by the condition $\zeta_{1}+\zeta_{2}+\zeta_{3}=2 \pi k$ for some $k \in \mathbb{Z}$. A general configuration can be parametrized as

$$
\phi_{\alpha}^{\prime}=\mathrm{e}^{\mathrm{i} \zeta_{\alpha}}\left(\boldsymbol{s}_{\alpha}^{\prime}+h_{\alpha}\right),
$$

with $h_{\alpha}, \alpha=1,2,3$ real scalar fields. The fourth physical degree of freedom is encoded into the $\mathrm{U}(1)_{\text {diag }}$ invariant combination $\theta=\zeta_{1}+\zeta_{2}+\zeta_{3}$. The Higgs potential is then

$$
\begin{aligned}
V^{\mathrm{YM}}\left(h_{1}, h_{2}, h_{3}, \theta\right) & \\
= & 4 g^{2} \sum_{\alpha=1}^{3}\left(2 h_{\alpha}^{2}\left(2 \varsigma_{\alpha}^{\prime}+h_{\alpha}\right)^{2}+\left(s_{1}^{\prime} \varsigma_{2}^{\prime} \varsigma_{3}^{\prime}\right)^{2} \frac{\left(s_{\alpha}^{\prime}+h_{\alpha}\right)^{2}}{\varsigma_{\alpha}^{\prime 4}}\right. \\
& +\left(s_{\alpha}^{\prime}+h_{\alpha}\right)^{2}\left(s_{\alpha+1}^{\prime}+h_{\alpha+1}\right)^{2} \\
& \left.-\frac{2 s_{1}^{\prime} \varsigma_{2}^{\prime} \varsigma_{3}^{\prime}\left(s_{1}^{\prime}+h_{1}\right)\left(s_{2}^{\prime}+h_{2}\right)\left(s_{3}^{\prime}+h_{3}\right)}{\varsigma_{\alpha}^{\prime 2}} \cos \theta\right) .
\end{aligned}
$$

Expanding (4.23) to second order in $h_{\alpha}$ and setting $\theta$ to zero, the Higgs mass matrix $\boldsymbol{m}$ is read off as 


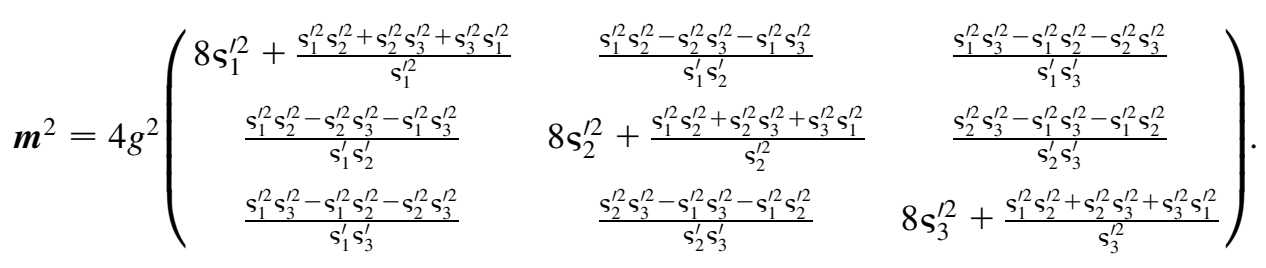

The eigenvalues of (4.24) at generic points $s_{\alpha}^{\prime}$ of the moduli space are symmetric under permutations of the indices $\alpha=1,2,3$, consistent with the center $\mathbb{Z}_{3}$ symmetry of the weight lattices $W^{k, l}$, but the explicit expressions are not very instructive. At the nearly Kähler and Kähler loci they reduce to

Nearly Kähler: $m_{1}=m_{2}=\frac{\sqrt{3}}{R}, \quad m_{3}=\frac{3}{2 R}$,

$$
\text { Kähler: } m_{ \pm}=\frac{\sqrt{\frac{9 \pm \sqrt{5}}{2}}}{R}, \quad m_{3}=\frac{\sqrt{5}}{R} \text {. }
$$

Incorporating the torsional Chern-Simons coupling with (4.19) is easily seen to yield a qualitatively similar mass matrix.

The fourth physical Higgs degree of freedom associated with the scalar field $\theta$ introduces degenerate vacua: The true vacuum state is a linear superposition of (4.21) with different integers $k \in \mathbb{Z}$ in the flatness condition $\theta=$ $\theta_{k}:=2 \pi k$ for the $\mathrm{U}(1)$ quiver lattice gauge theory. Let us look more closely at the effective field theory for this scalar. We turn off the gauge fields $A^{\alpha}$ and the Higgs fields $h_{\alpha}$, and use a $\mathrm{U}(1) \times \mathrm{U}(1)$ gauge transformation to choose a symmetric gauge in which all three phases $\zeta_{\alpha}$ are equal; this gauge choice preserves the $\mathbb{Z}_{3}$ symmetry under permutations of $s_{1}^{\prime}, s_{2}^{\prime}$ and $s_{3}^{\prime}$. Then we obtain from (4.11) and (4.23) the Lagrangian

$$
\mathcal{L}_{M}(\theta)=\frac{1}{2} \mathrm{~d} \vartheta \wedge \star \mathrm{d} \vartheta+\lambda(1-\cos \beta \vartheta) \wedge \star 1,
$$

where $\beta \vartheta=\theta$ with

$$
\beta=\frac{3}{\sqrt{2\left(s_{1}^{\prime 2}+s_{2}^{\prime 2}+s_{3}^{\prime 2}\right)}}=\frac{6 g}{\sqrt{M_{+}^{2}+M_{-}^{2}}}
$$

and

$$
\lambda=8 g^{2}\left(\varsigma_{1}^{\prime 2} \varsigma_{2}^{\prime 2}+\varsigma_{2}^{\prime 2} \varsigma_{3}^{\prime 2}+\varsigma_{3}^{\prime 2} \varsigma_{1}^{\prime 2}\right)=\frac{M_{+}^{2} M_{-}^{2}}{6 g^{2}} .
$$

On $M=\mathbb{R}^{1,1}$, this is just the Lagrangian of the sineGordon model [24]; in this case the gauge coupling $g$ has dimensions of mass and the metric moduli $s_{\alpha}^{\prime}$ are dimensionless. Expanding the potential in (4.26) for $\beta \rightarrow 0$ shows that the perturbative spectrum consists of scalar particles of mass

$$
M_{\mathrm{pert}}=\sqrt{\lambda} \beta=\frac{\sqrt{6} M_{+} M_{-}}{\sqrt{M_{+}^{2}+M_{-}^{2}}},
$$

which, like the gauge and Higgs boson masses (4.17) and (4.24), is independent of the gauge coupling $g^{2}$. On the other hand, this field theory admits well-known nonperturbative soliton solutions of mass

$$
M_{\mathrm{sol}}=\frac{\sqrt{\lambda}}{\beta}=\frac{\sqrt{6}}{36 g^{2}} M_{+} M_{-} \sqrt{M_{+}^{2}+M_{-}^{2}},
$$

which is dynamically determined by the induced gauge theory. Since all soliton solutions $\theta=\theta(t, x)$ must approach a vacuum solution $\theta_{k}$ at spatial infinity $|x| \rightarrow \infty$, one can associate with each of them a topological charge

$$
\begin{aligned}
Q=\frac{1}{2 \pi} \int_{\mathbb{R}} \mathrm{d} x \frac{\partial \theta}{\partial x} & =\frac{\theta(t,+\infty)-\theta(t,-\infty)}{2 \pi}=k_{+}-k_{-} \\
\text {with } \quad k_{ \pm} & \in \mathbb{Z},
\end{aligned}
$$

corresponding to the conserved topological current $J=$ $\frac{1}{2 \pi} \star \mathrm{d} \theta$ with $\mathrm{d} \star J=\frac{1}{2 \pi} \mathrm{d}^{2} \theta=0$. Such a field configuration tunnels between the degenerate vacua $\theta_{k_{+}}$and $\theta_{k_{-}}$. Note that the critical coupling of the sine-Gordon model $\beta^{2}=8 \pi$ (where the renormalized coupling has a simple pole) corresponds to gauge boson masses $M_{ \pm}$with $M_{+}^{2}+$ $M_{-}^{2}=\frac{9 g^{2}}{2 \pi}$; at the nearly Kähler and Kähler loci (4.18) of the moduli space, the base $\mathbb{C} P^{1}$ radius $R$ may then be regarded as being dynamically induced and is inversely proportional to the gauge coupling $g$; i.e. the large radius corresponds to the perturbative regime of the induced Yang-Mills-Higgs theory.

$N>3$. - Now we consider the case $p>1$. We start with $3 p^{2}$ complex Higgs fields, giving $6 p^{2}$ real scalar fields, $2 p^{2}$ of which are absorbed by the Higgs mechanism, thus leaving $4 p^{2}$ physical Higgs fields.

The physical Higgs masses can be obtained by using a polar decomposition of the Higgs fields $\phi_{\alpha}^{\prime}$ : Any square complex matrix $\phi$ can be uniquely decomposed into a unitary matrix $U$ and a Hermitian matrix $H$ as $\phi=U H$. To compute the masses it is sufficient to use constant matrices, thus minimizing the Higgs field kinetic energy, so we can expand about the vacuum solution and write

$$
\phi_{\alpha}^{\prime}=V_{\alpha}\left(\mathbf{s}_{\alpha}^{\prime} \mathbf{1}_{p}+h_{\alpha}\right)
$$

with $h_{\alpha}$ Hermitian. We are free to use a $\mathrm{U}(p) \times \mathrm{U}(p)$ gauge transformation to choose a gauge in which all three fields $V_{\alpha}$ are equal, $V_{\alpha}=\boldsymbol{U}$ for $\alpha=1,2,3$, while at the same time leaving the diagonal subgroup $\mathrm{U}(p)_{\text {diag }}$ intact. The physical degrees of freedom are now the $3 p^{2}$ fields in the Hermitian matrices $h_{\alpha}$ together with the $p^{2}$ angular 
variables in the Hermitian matrix $\boldsymbol{\theta}$ defined by $\boldsymbol{U}^{3}=$ : $\exp (\mathrm{i} \boldsymbol{\theta})$. The Higgs potential is now much more involved than in the Abelian case, since the fields $h_{\alpha}$ do not commute with $\boldsymbol{\theta}$ in general. Nevertheless, one can easily expand it to second order in $h_{\alpha}$ about the vacuum solution with $\boldsymbol{U}^{3}=\mathbf{1}_{p}$ to find the mass matrix $\boldsymbol{m}^{2} \otimes \mathbf{1}_{p}$, with $\boldsymbol{m}^{2}$ the mass matrix of the Abelian case (4.24).

The $p^{2}$ angular variables in the Hermitian matrix field $\boldsymbol{\theta}$ lead to a very interesting vacuum structure, in analogy to that of the sine-Gordon solitons for the Abelian case above. In particular, with all other fields turned off there is a matrix Higgs potential given by $\lambda \operatorname{Tr}_{p}\left(\mathbf{1}_{p}-\cos \boldsymbol{\theta}\right)$ for the symmetric gauge choice $V_{\alpha}=\boldsymbol{U}=\exp (\mathrm{i} \boldsymbol{\theta} / 3), \alpha=1,2$, 3 ; gauge equivalence classes of vacuum states are obtained by setting the eigenvalues of $\boldsymbol{\theta}$ to $\boldsymbol{\theta}_{i}=\boldsymbol{\theta}_{i, k_{i}}:=2 \pi k_{i}$ for $k_{i} \in \mathbb{Z}, i=1, \ldots, p$. On $M=\mathbb{R}^{1,1}$, soliton field configurations are parametrized by a sine-Gordon soliton $\boldsymbol{\theta}_{i}$ for each $i=1, \ldots, p$, together with an element $P \in S_{p}$ of the Weyl group of $\mathrm{U}(p)_{\text {diag }}$, which permutes the eigenvalues of the matrix $\boldsymbol{\theta}$; such a soliton carries a topological charge vector $\vec{Q} \in \mathbb{Z}^{p}$ with entries

$$
\vec{Q}_{i}=\frac{\boldsymbol{\theta}_{i, k_{+}}-\boldsymbol{\theta}_{P(i), k_{-}}}{2 \pi}
$$

interpolating among the $p$ families of infinitely degenerate vacuum sectors.

\section{Flat connections in quiver lattice gauge theory}

For any pair of non-negative integers $(k, l)$, the dimensional reduction of Yang-Mills gauge theory on $\mathcal{M}$ to a Yang-Mills-Higgs theory on $M$ associated with the irreducible $\mathrm{SU}(3)$ representation $\hat{V}^{k, l}$ can be described using the formulas of Appendix B; the general expression for the induced quiver gauge theory action on $M$ is rather lengthy and not very informative. We will therefore satisfy ourselves with studying the solutions of the vacuum equations with constant Higgs fields and vanishing gauge potentials. The Higgs potential is a sum of squares, one for each term multiplying the $\tilde{\Theta} \wedge \tilde{\Theta}$ forms in (B1)-(B3). The vacuum equations are thus obtained by setting each of these terms to zero, and after rescaling the Higgs fields analogously to (4.10) they read as

$$
\begin{aligned}
& \sum_{ \pm}\left[\frac{n \mp q+1 \pm 1}{n+1} \lambda_{k, l}^{ \pm}(n, m)^{2}\left(\boldsymbol{s}_{1}^{\prime 2} \mathbf{1}_{N_{(q, m)_{n}}}-\phi_{(q, m)_{n}}^{1}{ }^{( \pm) / \dagger} \phi_{(q, m)_{n}}^{1}{ }^{( \pm) \prime}\right)\right. \\
& \left.-\frac{n \mp q+1 \mp 1}{n+1 \mp 1} \lambda_{k, l}^{ \pm}(n \mp 1, m-3)^{2}\left(\boldsymbol{s}_{1}^{\prime 2} \mathbf{1}_{N_{(q, m)_{n}}}-\phi_{(q+1, m-3)_{n \mp 1}}^{1}{ }^{( \pm) \prime} \phi_{(q+1, m-3)_{n \mp 1}}^{1}{ }^{( \pm) / \dagger}\right)\right]=0, \\
& \sum_{ \pm}\left[\frac{n \mp q+1 \pm 1}{n+1 \pm 1} \lambda_{k, l}^{\mp}(n \pm 1, m-3)^{2}\left(\varsigma_{2}^{\prime 2} \mathbf{1}_{N_{(q, m)_{n}}}-\phi_{(q, m)_{n}}^{2}{ }^{( \pm) / \dagger} \phi_{(q, m)_{n}}^{2}{ }^{( \pm) /}\right)\right. \\
& \left.-\frac{n \mp q+1 \mp 1}{n} \lambda_{k, l}^{\mp}(n, m)^{2}\left(s_{2}^{\prime 2} \mathbf{1}_{N_{(q, m) n}}-\phi_{(q+1, m+3)_{n \mp 1}}^{2}{ }^{( \pm) \prime} \phi_{(q+1, m+3)_{n \mp 1}}^{2}{ }^{( \pm) / \dagger}\right)\right]=0, \\
& (n-q)(n+q+2)\left(s_{3}^{\prime 2} \mathbf{1}_{N_{(q, m)_{n}}}-\phi_{(q, m)_{n}}^{3 \prime} \phi_{(q, m)_{n}}^{3 \prime}\right)-(n+q)(n-q+2)\left(s_{3}^{\prime 2} \mathbf{1}_{N_{(q, m)_{n}}}-\phi_{(q-2, m)_{n}}^{3 \prime} \phi_{(q-2, m)_{n}}^{3 \prime}\right)=0 \text {, }
\end{aligned}
$$

together with

$$
\begin{aligned}
& \sqrt{\frac{\left((n+1 \pm 1)^{2}-q^{2}\right)(n \pm q+1 \pm 1)}{2(n+1)}}\left(\boldsymbol{s}_{2}^{\prime} \boldsymbol{s}_{3}^{\prime} \phi_{(q, m)_{n}}^{1}{ }^{( \pm) \prime}-\boldsymbol{s}_{1}^{\prime} \phi_{(q-1, m+3)_{n \pm 1}}^{3 \prime} \phi_{(q+1, m+3)_{n \pm 1}}^{\dagger(+\mp) \prime}\right) \\
& -\sqrt{\frac{(n \pm q+1 \mp 1)(n-q+2)(n+q)}{2(n+1)}}\left(\varsigma_{2}^{\prime} \varsigma_{3}^{\prime} \phi_{(q, m)_{n}}^{1}{ }^{( \pm) \prime}-\varsigma_{1}^{\prime} \phi_{(q-1, m+3)_{n \pm 1}}^{2(\mp) \prime}{ }^{\dagger} \phi_{(q-2, m)_{n}}^{3 \prime}{ }^{\dagger}\right)=0, \\
& \sqrt{\frac{\left((n+1 \pm 1)^{2}-q^{2}\right)(n \pm q+1 \pm 1)}{2(n+1 \pm 1)}}\left(\varsigma_{1}^{\prime} \varsigma_{3}^{\prime} \phi_{(q, m)_{n}}^{2( \pm) \prime}-\varsigma_{2}^{\prime} \phi_{(q-1, m-3)_{n \pm 1}}^{1}{ }^{(\mp) / \dagger} \phi_{(q-2, m)_{n}}^{3 /}{ }^{\dagger}\right) \\
& -\sqrt{\frac{(n \pm q+1 \mp 1)(n-q+2)(n+q)}{2(n+1 \pm 1)}}\left(\varsigma_{1}^{\prime} s_{3}^{\prime} \phi_{(q, m)_{n}}^{2( \pm) \prime}-\varsigma_{2}^{\prime} \phi_{(q-1, m-3)_{n \pm 1}}^{3 \prime}{ }^{\dagger} \phi_{(q+1, m-3)_{n \pm 1}}^{1(\mp) \prime}{ }^{\dagger}\right)=0, \\
& \sum_{ \pm}\left[\lambda_{k, l}^{ \pm}(n \mp 1, m-3)^{2}\left(\boldsymbol{s}_{1}^{\prime} \varsigma_{2}^{\prime} \phi_{(q, m)_{n}}^{3 \prime}-\varsigma_{3}^{\prime} \phi_{(q+2, m)_{n}}^{2(\mp))}{ }^{\dagger} \phi_{(q+1, m-3)_{n \mp 1}}^{1( \pm) l}{ }^{\dagger}\right)-\lambda_{k, l}^{ \pm}(n, m)^{2}\left(\varsigma_{1}^{\prime} \varsigma_{2}^{\prime} \phi_{(q, m)_{n}}^{3 \prime}\right.\right. \\
& \left.\left.-\varsigma_{3}^{\prime} \phi_{(q+2, m)_{n}}^{1( \pm) \prime} \phi_{(q+1, m+3)_{n \pm 1}}^{2(\mp)}\right)\right]=0,
\end{aligned}
$$

and 
$\phi_{(q, m)_{n}}^{3 \prime} \phi_{(q, m)_{n}}^{1}{ }^{( \pm) / \dagger}=\phi_{(q+2, m)_{n}}^{1}{ }^{( \pm) / \dagger} \phi_{(q-1, m+3)_{n \pm 1}}^{3 /}$,

$\phi_{(q, m)_{n}}^{3 /} \phi_{(q, m)_{n}}^{2}{ }^{( \pm) / \dagger}=\phi_{(q+2, m)_{n}}^{2}{ }^{( \pm) / \dagger} \phi_{(q-1, m-3)_{n \pm 1}}^{3 /}$,

$\phi_{(q+2, m)_{n}}^{(\mp) \prime} \phi_{(q+2, m)_{n}}^{( \pm) \prime \dagger}$

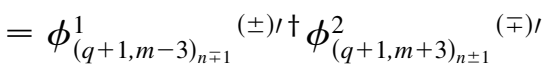

for each weight $(q, m)_{n} \in W^{k, l}$.

When $N_{(q, m)_{n}}=p$ for all weights $(q, m)_{n} \in W^{k, l}$, the gauge symmetry reduction is given by

$$
\mathrm{U}(N) \rightarrow \mathrm{U}(p)^{d^{k, l}} \quad \text { with } \quad N=p d^{k, l},
$$

where $d^{k, l}=\left|W^{k, l}\right|$ is the dimension (A10) of the irreducible $\mathrm{SU}(3)$ representation $\hat{V}^{k, l}$. In this instance an explicit solution of (4.34) is given by

$$
\begin{aligned}
\phi_{(q, m)_{n}}^{1}{ }^{( \pm) \prime} & =\varsigma_{1}^{\prime} V_{(q, m)_{n}}^{1}{ }^{( \pm)}, \\
\phi_{(q, m)_{n}}^{2}{ }^{( \pm) \prime} & =\varsigma_{2}^{\prime} V_{(q, m)_{n}}^{2}{ }^{( \pm)} \quad \text { and } \\
\phi_{(q, m)_{n}}^{3 \prime} & =\varsigma_{3}^{\prime} V_{(q, m)_{n}}^{3}{ }^{3 \prime}
\end{aligned}
$$

with unitary matrices $V_{(q, m)_{n}}^{1}{ }^{( \pm)}, V_{(q, m)_{n}}^{2}{ }^{( \pm)}, V_{(q, m)_{n}}^{3} \in \mathrm{U}(p)$ for each $(q, m)_{n} \in W^{k, l}$. Substituting into (4.36) yields the commutation relations

$$
\begin{aligned}
V_{(q+2, m)_{n}}^{1}{ }^{( \pm)} V_{(q, m)_{n}}^{3} & =V_{(q-1, m+3)_{n \pm 1}}^{3} V_{(q, m)_{n}}^{1}{ }^{( \pm)}, \\
V_{(q+2, m)_{n}}^{2}{ }^{( \pm)} V_{(q, m)_{n}}^{3} & =V_{(q-1, m-3)_{n \pm 1}}^{3} V_{(q, m)_{n}}^{2}{ }^{( \pm)}, \\
V_{(q+1, m-3)_{n \mp 1}{ }^{( \pm)} V_{(q+2, m)_{n}}^{(\mp)}}^{2} & =V_{(q+1, m+3)_{n \pm 1}{ }^{(\mp)} V_{(q+2, m)_{n}}{ }^{( \pm)},}^{2}
\end{aligned}
$$

and then (4.35) leads to the conditions

$$
V_{(q-1, m-3)_{n \pm 1}}^{1}{ }^{(\mp)} V_{(q, m)_{n}}^{2}{ }^{( \pm)} V_{(q-2, m)_{n \pm 1}}^{3}=\mathbf{1}_{p}
$$

for each $(q, m)_{n} \in W^{k, l}$. In contrast to the Bogomol'nyiPrasad-Sommerfeld equations derived in [12] [which do not involve the curvature matrix elements (B3)], the requirements (4.40) are much stronger than the set of relations (4.35) of the double quiver $\bar{Q}^{k, l}$ associated with $\hat{V}^{k, l}$; as before they specify a flat connection of the $\mathrm{U}(p)$ lattice gauge theory on the finite quiver lattice $Q^{k, l}$, which is a tessellation of the plane $\mathbb{R}^{2}$ by equilateral triangles associated with the vector representations $\hat{V}^{0,1}$ and $\hat{V}^{1,0}$ of $\mathrm{SU}(3)$. Including a Chern-Simons coupling at the special value (4.19) removes the conditions (4.36) and modifies (4.34) into a single set of equations representing the moment map equations of $\bar{Q}^{k, l}[12]$.

See [[11], Sec. II B] for the explicit construction of the nodes and arrows for a generic quiver $Q^{k, l}$; below we use these results in the combinatorics of physical fields.

Triangular quivers. - To enumerate the physical degrees of freedom remaining after the dynamical symmetry breaking mechanism, we first consider the representations $\hat{V}^{k, 0}$ (and their complex conjugates $\hat{V}^{0, k}$ ) of dimension $d^{k, 0}=$ $\frac{1}{2}(k+1)(k+2)$. In this case the boundary of the weight diagram $W^{k, 0}$ is an inverted equilateral triangle, the weight states are all unique, and there are $k+1$ hypercharge levels (see Appendix A and Fig. 1). Starting at the lowest hypercharge eigenvalue $m=-2 k$, we tessellate the interior of the boundary triangle with equilateral triangles of the form (3.10) (and its inversion representing the quiver $Q^{1,0}$ ); at level $i$ there are $2 i-1$ triangles with $3 i$ edges in total for $i=$ $1, \ldots, k$. Hence the solution (4.38) involves $\frac{3}{2} k(k+1)$ unitary degrees of freedom, one for each link of the quiver lattice $Q^{k, 0}$. We can use a gauge transformation in the $\mathrm{U}(p)^{d^{k, 0}-1}$ subgroup of (4.37) for $l=0$ to set $d^{k, 0}-1$ of these lattice gauge fields all equal to a constant unitary matrix $\boldsymbol{U}$ on the lattice with $\boldsymbol{U}^{3}=\mathbf{1}_{p}$, and then solve for the remaining fields using the $k^{2}$ plaquette relations (4.40). Thus the solution (4.38) breaks the gauge symmetry of the $d$-dimensional field theory on $M$ to the diagonal subgroup $\mathrm{U}(p)_{\text {diag }}$, leaving in this case $\frac{1}{2} k(k+3) p^{2}$ massive gauge bosons (with physical masses proportional to $\frac{1}{R}$ at the Kähler and nearly Kähler loci of the moduli space) and $\frac{1}{2} k(5 k+3) p^{2}$ real physical Higgs fields. Of these scalar fields, $p^{2}$ of them reside in the $\mathrm{U}(p)_{\text {diag }}$ invariant Hermitian field $\boldsymbol{\theta}$ defined by $\boldsymbol{U}^{3}=: \exp (\mathrm{i} \boldsymbol{\theta})$, whose vacuum structure is qualitatively analogous to that associated with the antifundamental representation $\hat{V}^{0,1}$ from before.

For example, the quiver $Q^{2,0}$ associated with the sixdimensional representation $\hat{V}^{2,0}$ is

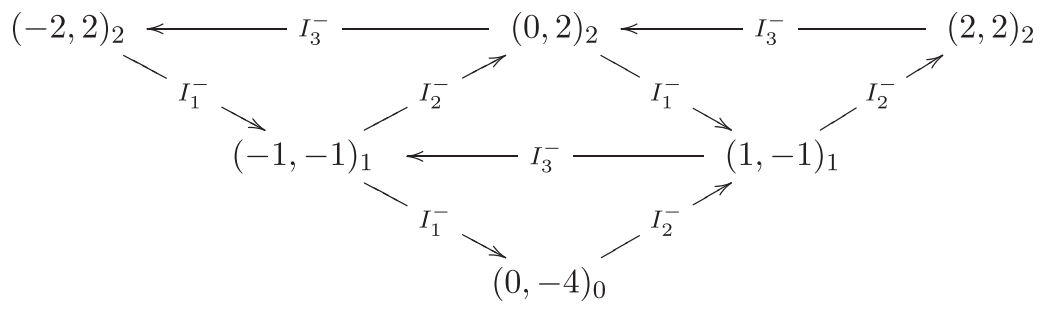

and the vacuum field content consists of $5 p^{2}$ massive gauge bosons plus $13 p^{2}$ real physical scalar fields.

Hexagonal quivers.-For a generic representation $\hat{V}^{k, l}$ with $k, l \neq 0, k \geq l$ (or its complex conjugate $\hat{V}^{l, k}$ ), the boundary of the weight diagram $W^{k, l}$ is a hexagon, symmetric about $(0,0) \in \mathbb{Z}^{2}$, with $k+1$ weights on the upper edge, $l+1$ weights on the lower edge, and $k+l+1$ hypercharge levels from the lower to the upper edge (see Fig. 2). The outer $l$ layers are all hexagonal, while the inner layer $l+1$ is triangular (and hence so are all of its interior 


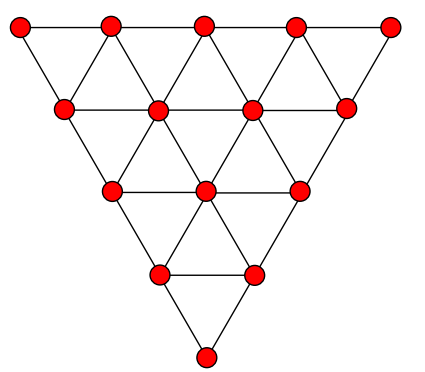

FIG. 1 (color online). Weight diagram $W^{4,0}$.

layers). The counting of physical degrees of freedom is further complicated now by the existence of degenerate weight states: States in the $i$ th hexagonal layer have multiplicity $i$ for $i=1, \ldots, l$, while states in the inverted triangular layers all have multiplicity $l+1$. In the corresponding quiver diagram, diagonal links around layers of fixed multiplicity $i$ are mapped to $i$ arrows between the nodes, and diagonal links from a layer with multiplicity $i$ to a layer with multiplicity $i+1$ are mapped to $2 i$ arrows; horizontal links always map to single arrows. Starting from the lowest hypercharge eigenvalue $m=-2 k-l$, the tip of the first inverted triangular layer starts $l$ levels up at $m=-2 k+2 l$. The enumeration within the interior triangle is thus that of the triangular representation $\hat{V}^{k-l, 0}$ as derived above, adjusted by the multiplicity factor $l+1$; in particular, it contains $d^{k-l, 0}=\frac{1}{2}(k-l+1)(k-l+2)$ nodes and

$$
\begin{aligned}
& \frac{3}{2}(k-l)(k-l+1)(l+1)-\frac{1}{2} l(k-l)(k-l+1) \\
& \quad=\frac{1}{2}(k-l)(k-l+1)(2 l+3)
\end{aligned}
$$

complex Higgs fields, where the subtraction compensates the overcounting of horizontal arrows in the quiver diagram. The boundary of the weight diagram is generated by $k$ applications of the $\mathrm{SU}(3)$ operators $I_{\alpha}^{-}$and $l$ applications of $I_{\bar{\alpha}}^{+}$for $\alpha=1,2,3$; hence there are $3(k+l)$ boundary nodes (each of multiplicity one). The $i$ th hexagonal layer contains $3(k+l-2 i+2)$ weights, each of multiplicity $i$ for $i=1, \ldots, l$. By suitably compensating the enumeration of horizontal arrows and the two "corner" nodes, it is straightforward to see that there are $3 i(k+l-2 i+2)-$ $(k+l-2 i+2)(i-1)$ arrows around layer $i, 3(k+l-$ $2 i+2)-(k+l-2 i+4)$ additional horizontal arrows, and $8 i(k+l-2 i+1)$ diagonal arrows to layer $i+1$. Adding everything, there are altogether

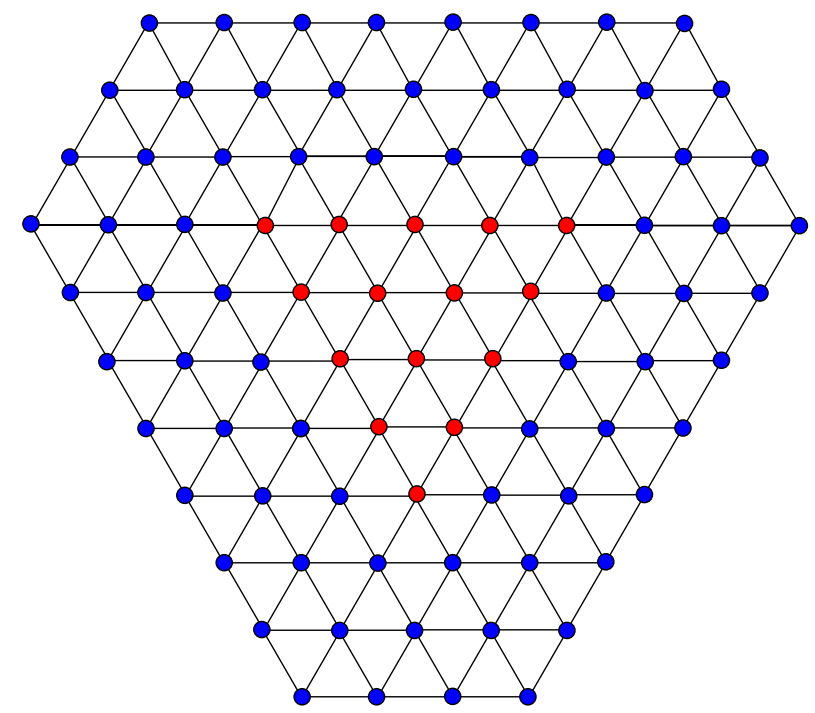

FIG. 2 (color online). Weight diagram $W^{7,3}$ (multiplicities are not shown).

$$
\sum_{i=1}^{l}((10 i+3)(k+l-2 i+2)-2(4 i+1))
$$

complex Higgs fields on the outer $l$ hexagonal layers. The total number of unitary matrices parametrizing the vacuum solution (4.38) is then the sum of (4.42) and (4.43), which is

$$
\frac{1}{2} k^{2}(2 l+3)+\frac{3}{2} k\left(2 l^{2}+4 l+1\right)-\frac{1}{6} l(l+1)(4 l-13) .
$$

Again, we eliminate these lattice gauge fields in favor of a unitary matrix $\boldsymbol{U}$ with $\boldsymbol{U}^{3}=\mathbf{1}_{p}$ by using a $\mathrm{U}(p)^{d^{k, l}-1}$ gauge transformation and the plaquette relations (4.40). The gauge symmetry is broken to $\mathrm{U}(p)_{\text {diag }}$, leaving $\left(d^{k, l}-1\right) p^{2}$ massive gauge bosons and a total of

$$
\frac{1}{2} k^{2}(3 l+5)+\frac{1}{2} k\left(11 l^{2}+20 l+3\right)-\frac{1}{6} l\left(8 l^{2}-15 l-17\right)
$$

real physical Higgs fields.

For example, the quiver $Q^{1,1}$ associated with the eightdimensional adjoint representation $\hat{V}^{1,1}$ is

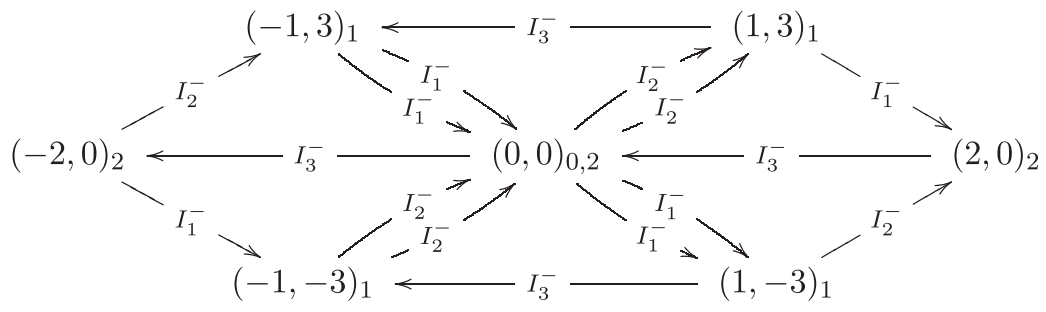


leading to $7 p^{2}$ massive gauge fields and $25 p^{2}$ real physical scalar fields.

\section{INDUCED YUKAWA INTERACTIONS OF SYMMETRIC FERMIONS}

In this section we will describe the SU(3)-equivariant dimensional reduction of the Yang-Mills-Dirac theory on $\mathcal{M}=M \times \mathbb{F}_{3}$ for invariant spinor fields over the flag manifold $\mathbb{F}_{3}$, and the induced Yang-Mills-Higgs-Dirac theory on $M$. We will focus particular attention to the possible emergence of Yukawa couplings between the Higgs and fermion fields.

\section{A. Reduction of the Dirac action}

Consider the minimally coupled Dirac Lagrangian $(d+6)$-form on $\mathcal{M}$ given by

$$
\mathcal{L}_{\mathcal{M}}^{\mathrm{D}}=i \Psi^{\dagger} \wedge \tilde{\star} \mathcal{D} \Psi
$$

on the space of massless $\mathrm{L}^{2}$ spinors (3.17), where the fermion field $\Psi$ has canonical dimension $\frac{1}{2}(d+5)$. For simplicity, we take a Euclidean signature metric on $M$; for the Lorentzian signature the adjoint spinor $\Psi^{\dagger}$ should be replaced with the appropriate Lorentzian adjoint $\bar{\Psi}$. We also assume for definiteness that the spinor field $\Psi$ transforms under the fundamental representation of the $\mathrm{U}(N)$ gauge group, but other fermion representations can be similarly treated. For representations $\hat{V}^{k, l}$ that give rise to a nonzero index for the Dirac operators on $\mathbb{F}_{3}$, and hence to harmonic spinors, dimensional reduction of this Lagrangian yields a nontrivial fermionic field theory on $M$ coupled to the Yang-Mills-Higgs theory of Sec. IV.

By integrating this Lagrangian over the coset space $\mathbb{F}_{3}$, we arrive at a dimensionally reduced Lagrangian $d$-form

$$
\int_{\mathbb{E}_{3}} \mathcal{L}_{\mathcal{M}}^{\mathrm{D}}=\mathcal{L}_{M}^{\mathrm{D}}+\mathcal{L}_{M}^{\mathrm{Y}}+\mathcal{L}_{M}^{\mathrm{Y}}{ }^{\dagger}
$$

on $M$. The second term from (3.22) vanishes on harmonic spinor fields on $\mathbb{F}_{3}$, while the first term yields a series of massless twisted Dirac kinetic terms for the various fermion fields on $M$; with the same rescalings of the bosonic fields and the metric moduli as in Sec. IV, using the orthogonality relations (2.44) this gives

$$
\begin{aligned}
\mathcal{L}_{M}^{\mathrm{D}}= & i \sum_{(q, m)_{n} \in W_{+}^{k, l}} \sum_{\ell=1}^{v_{q, m}} \psi_{(q, m)_{n} ; \ell} \ell^{\dagger} \wedge \star\left(\not \not_{M}+g \not \mathcal{A}^{(q, m)_{n}}\right) \psi_{(q, m)_{n} ; \ell} \\
& +i \sum_{(q, m)_{n} \in W_{-}^{k, l}} \sum_{\ell=1}^{\left|\nu_{q, m}\right|} \eta_{(q, m)_{n} ; \ell} \ell^{\dagger} \wedge \star\left(\not \partial_{M}+g \not \mathscr{A}^{\left.\prime(q, m)_{n}\right)}\right. \\
& \times \eta_{(q, m)_{n} ; \ell .}
\end{aligned}
$$

The fermion fields $\psi_{(q, m)_{n} ; \ell}$ and $\eta_{(q, m)_{n} ; \ell^{\prime}}$ transform in the fundamental representation of $\mathrm{U}\left(N_{(q, m)_{n}}\right)$ for each $\ell=$ $1, \ldots, \nu_{q, m}$ and $\ell^{\prime}=1, \ldots,\left|\nu_{q, m}\right|$, respectively.
Zero modes of the Dirac operator on $\mathbb{F}_{3}$ can also give rise to Yukawa couplings when the theory is reduced to $M$. Upon reduction to $M$, Yukawa couplings between $\psi_{(q, m)_{n} ; \ell}$ and $\eta_{(q, m)_{n} ; \ell}$ can arise from integrating the offdiagonal terms from the operator (3.24), involving the Higgs fields, in

$\Psi^{\dagger} \wedge \tilde{\star} \widetilde{y} \Psi=\Psi^{-\dagger} \wedge \tilde{\star} \tilde{y} \Psi^{+}+\Psi^{+\dagger} \wedge \tilde{\star} \tilde{y} \Psi^{-}$

over $\mathbb{F}_{3}$, and they depend crucially on the zero mode structure. The projectors $\hat{\Pi}_{(q, m)_{n}}$ pick out specific zero modes in the expansions (3.17). Using the explicit matrix elements from (A17), upon integration over $\mathbb{F}_{3}$ only the singlet parts of the fermion bilinears $\chi_{q^{\prime}, m^{\prime} ; \ell^{\prime}}^{ \pm} \tilde{\gamma}^{\alpha} \chi_{q, m ; \ell}^{\mp}$ can survive and generate Yukawa coupling coefficients,

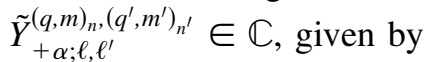

$$
\begin{aligned}
& \tilde{Y}^{(q, m)_{n},(q-1, m+3)_{n \pm 1}} \\
& +1 ; \ell, \ell^{\prime} \\
& =\sqrt{\frac{n \mp q+1 \pm 1}{2(n+1)}} \lambda_{k, l}^{ \pm}(n, m) \int_{\mathbb{F}_{3}} \frac{\tilde{\omega}^{\wedge 3}}{3 !} \chi_{q, m ; \ell}^{+} \tilde{\gamma}^{1} \chi_{q-1, m+3 ; \ell^{\prime}}^{-}, \\
& \tilde{Y}_{+2 \cdot \ell, \ell^{\prime}}^{(q, m)_{n},(q-1, m-3)_{n \pm 1}} \\
& =\sqrt{\frac{n \mp q+1 \pm 1}{2(n+1 \pm 1)}} \lambda_{k, l}^{\mp}(n \pm 1, m-3) \\
& \times \int_{\mathbb{F}_{3}} \frac{\tilde{\omega}^{\wedge 3}}{3 !} \chi_{q, m ; \ell}^{+} \tilde{\gamma}^{2} \chi_{q-1, m-3 ; \ell^{\prime}}^{-}, \\
& \tilde{Y}_{+3 \cdot \ell, \ell^{\prime}}^{(q, m)_{n},(q+2, m)_{n}} \\
& =\frac{1}{2} \sqrt{(n-q)(n+q+2)} \int_{\mathbb{F}_{3}} \frac{\tilde{\omega}^{\wedge 3}}{3 !} \chi_{q, m ; \ell}^{+} \tilde{\gamma}^{3} \chi_{q+2, m ; \ell^{\prime}}^{-},
\end{aligned}
$$

on $M$, plus a completely analogous set of coupling co-

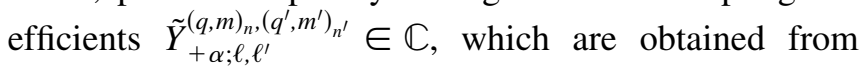
(5.5) by interchanging chiral and antichiral spinor labels $\chi^{+} \leftrightarrow \chi^{-}$, keeping the same coefficients,

$$
\begin{aligned}
\tilde{Y}_{-1 ; \ell, \ell^{\prime}}^{(q, m)_{n},(q-1, m+3)_{n \pm 1}}= & \sqrt{\frac{n \mp q+1 \pm 1}{2(n+1)}} \lambda_{k, l}^{ \pm}(n, m) \\
& \times \int_{\mathbb{F}_{3}} \frac{\tilde{\omega}^{\wedge 3}}{3 !} \chi_{q, m ; \ell}^{-} \tilde{\gamma}^{1} \chi_{q-1, m+3 ; \ell^{\prime}}^{+}, \\
\tilde{Y}_{-2 ; \ell, \ell^{\prime}}^{(q, m)_{n},(q-1, m-3)_{n \pm 1}}= & \sqrt{\frac{n \mp q+1 \pm 1}{2(n+1 \pm 1)}} \lambda_{k, l}^{\mp}(n \pm 1, m-3) \\
& \times \int_{\mathbb{F}_{3}} \frac{\tilde{\omega}^{\wedge 3}}{3 !} \chi_{q, m ; \ell}^{-} \tilde{\gamma}^{2} \chi_{q-1, m-3 ; \ell^{\prime}}^{+}, \\
\tilde{Y}_{-3 ; \ell, \ell^{\prime}}^{(q, m)_{n},(q+2, m)_{n}}= & \frac{1}{2} \sqrt{(n-q)(n+q+2)} \\
& \times \int_{\mathbb{F}_{3}} \frac{\tilde{\omega}^{\wedge 3}}{3 !} \chi_{q, m ; \ell}^{-} \tilde{\gamma}^{3} \chi_{q+2, m ; \ell^{\prime}}^{+}
\end{aligned}
$$


Note that a Yukawa coupling between two weights connected by a quiver arrow $\left(q^{\prime}, m^{\prime}\right)_{n^{\prime}} \rightarrow(q, m)_{n}$ can arise only if the corresponding indices are of opposite sign, due to the change in spinor chirality induced by multiplication with the $\gamma$ matrices, $\tilde{\gamma}^{\alpha}$. In Fig. 3 we depict some examples of the Dirac index associated with some low values of $k$ and $l$. Using $W_{+}^{k, l} \cap W_{-}^{k, l}=\emptyset$, the $d$-dimensional Yukawa interactions on $M$ are then given generically by

$$
\begin{aligned}
& \mathcal{L}_{M}^{\mathrm{Y}}=2 i g \sum_{(q, m)_{n} \in W_{+}^{k, l}} \sum_{\ell, \ell^{\prime}=1}^{\nu_{q, m}}\left(\sum_{ \pm} \tilde{Y}_{+1 ; \ell, \ell^{\prime}}^{(q, m)_{n},(q-1, m+3)_{n \pm 1}} \psi_{(q, m)_{n} ; \ell^{\dagger}}^{\dagger} \wedge \star \phi_{(q, m)_{n}}^{1}{ }^{( \pm) / \dagger} \breve{\psi}_{(q-1, m+3)_{n \pm 1} ; \ell^{\prime}}\right. \\
& \left.+\sum_{ \pm} \tilde{Y}_{+2 ; \ell, \ell^{\prime}}^{(q, m)_{n},(q-1, m-3)_{n \pm 1}} \psi_{(q, m)_{n} ; \ell^{\dagger}}^{\dagger} \wedge \star \phi_{(q, m)_{n}}^{2}{ }^{( \pm) / \dagger} \breve{\psi}_{(q-1, m-3)_{n \pm 1} ; \ell^{\prime}}+\tilde{Y}_{+3 ; \ell, \ell^{\prime}}^{(q, m)_{n},(q+2, m)_{n}} \psi_{(q, m)_{n} ; \ell^{\dagger}}^{\dagger} \wedge \star \phi_{(q, m)_{n}}^{3 \prime}{ }^{\dagger} \breve{\psi}_{(q+2, m)_{n} ; \ell^{\prime}}\right) \\
& +2 i g \sum_{(q, m)_{n} \in W_{-}^{k, l}} \sum_{\ell, \ell^{\prime}=1}^{\left|\nu_{q, m}\right|}\left(\sum_{ \pm} \tilde{Y}_{-1 ; \ell, \ell^{\prime}}^{(q, m)_{n},(q-1, m+3)_{n \pm 1}} \breve{\psi}_{(q, m)_{n} ;} \ell^{\dagger} \wedge \star \phi_{(q, m)_{n}}^{1}{ }^{( \pm) / \dagger} \psi_{(q-1, m+3)_{n \pm 1} ; \ell^{\prime}}\right. \\
& \left.+\sum_{ \pm} \tilde{Y}_{-2 ; \ell, \ell^{\prime}}^{(q, m)_{n},(q-1, m-3)_{n \pm 1}} \breve{\psi}_{(q, m)_{n} ; \ell^{\dagger}} \wedge \star \phi_{(q, m)_{n}}^{2}{ }^{( \pm) / \dagger} \psi_{(q-1, m-3)_{n \pm 1} ; \ell^{\prime}}+\tilde{Y}_{-3 ; \ell, \ell^{\prime}}^{(q, m)_{n},(q+2, m)_{n}} \breve{\psi}_{(q, m)_{n} ; \ell} \ell^{\dagger} \wedge \star \phi_{(q, m)_{n}}^{3 \prime}{ }^{\dagger} \psi_{(q+2, m)_{n} ; \ell^{\prime}}\right) \text {, }
\end{aligned}
$$

where $\breve{\psi}_{(q, m)_{n} ; \ell}:=\gamma \eta_{(q, m)_{n} ; \ell}$, together with its Hermitian conjugate in (5.2).

Without an explicit construction of the harmonic spinors we cannot evaluate the Yukawa coupling coefficients (5.5) and (5.6), nor indeed say which of them will be generally nonzero for a given choice of SU(3) representation $\hat{V}^{k, l}$. In the case of equivariant dimensional reduction of the YangMills-Dirac theory over the projective spaces $\mathbb{C} P^{n}$ with $n=1,2$, which are Kähler manifolds, it was observed in $[13,14]$ that Yukawa couplings arise only in the contributions from harmonic spinors that are constant on the coset space; for $\mathbb{C} P^{1}=\mathrm{SU}(2) / \mathrm{U}(1)$ such spinors exist in every even-dimensional irreducible representation of $\mathrm{SU}(2)$, while for $\mathbb{C} P^{2}$ there is a unique choice of $\operatorname{spin}^{c}$ structure for each irreducible $\mathrm{SU}(3)$ representation $\hat{V}^{k, l}$, which accommodates constant spinor harmonics. Moreover, when they exist, the constant spinor harmonics are unique and hence lie in states of index \pm 1 . In the following we will demonstrate that an analogous construction applies to the nearly Kähler coset space $\mathbb{F}_{3}$, except that we shall also find constant spinor harmonics for a class of nodes of index zero. We will furthermore compare the Yukawa interactions (5.7) at different points of the moduli space.

\section{B. Symmetric spinors of torsion class $W_{1}$}

We will begin by classifying the $\mathrm{SU}(3)$ representations $\hat{V}^{k, l}$ that permit nonvanishing Yukawa couplings (5.7), and lead to dynamical mass generation for the fermion fields via spontaneous symmetry breaking, at the locus $\sigma=1$ of the moduli space; recall that this surface contains the nearly Kähler point (2.29). In this case the Dirac operator $\not_{\mathbb{E}_{3}}^{1}$ from (2.36) is associated with the canonical connection (2.23). We will explicitly construct harmonic spinors of the corresponding Dirac operators (2.35).

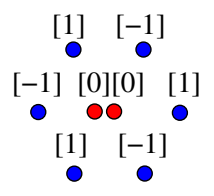

$(\mathbf{k}, \mathbf{l})=(\mathbf{1}, \mathbf{1})$

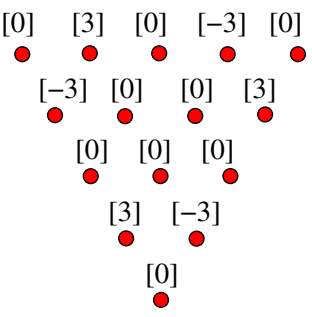

$(\mathrm{k}, \mathrm{l})=(4,0)$

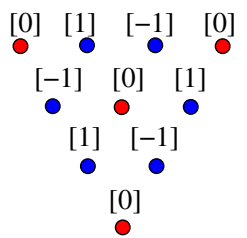

$(\mathbf{k}, \mathbf{l})=(\mathbf{3 , 0})$

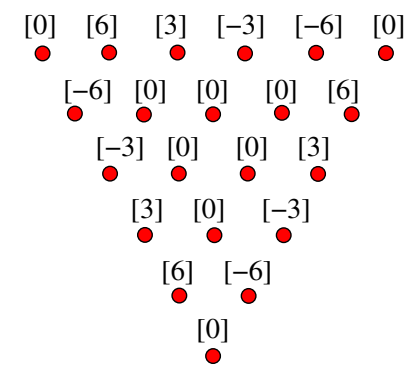

$(\mathbf{k}, \mathbf{l})=(\mathbf{5 , 0})$

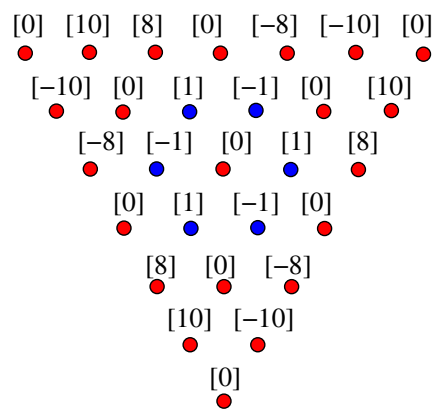

$(\mathrm{k}, \mathrm{l})=(6,0)$

FIG. 3 (color online). Nodes of lowest SU(3) weight diagrams accommodating harmonic spinors, with the index $\nu_{q, m}$ in square brackets attached to each weight $(q, m)_{n} \in W^{k, l}$. 
For this, we decompose the complex $(1,0)$-forms $\tilde{\Theta}^{\alpha}$ into an invariant, local real orthonormal basis $e^{a}, a=$ $1, \ldots, 6$ of the cotangent bundle $T^{*} \mathbb{F}_{3}$ as

$$
\tilde{\Theta}^{\alpha}=\frac{1}{2}\left(e^{2 \alpha-1}+\mathrm{i} e^{2 \alpha}\right)
$$

for $\alpha=1,2,3$. Similarly, we decompose the corresponding complex gamma matrices $\tilde{\gamma}^{\alpha}$ into Hermitian gamma matrices $\gamma^{a}, a=1, \ldots, 6$ as

$$
\tilde{\gamma}^{\alpha}=\frac{1}{2}\left(\gamma^{2 \alpha-1}+\mathrm{i} \gamma^{2 \alpha}\right)
$$

for $\alpha=1,2,3$; they obey the Clifford relations

$$
\left\{\gamma^{a}, \gamma^{b}\right\}=2 \delta^{a b} \mathbf{1}_{8}
$$

Then the canonical torsion 3-form can be expressed as

$H=-\frac{1}{4 \sqrt{3}} f_{a b c} e^{a b c}=\frac{1}{4}\left(e^{135}+e^{425}+e^{416}+e^{326}\right)$,

where generally $e^{a_{1} \ldots a_{r}}:=e^{a_{1}} \wedge \cdots \wedge e^{a_{r}}$ with $a_{i}=$ $1, \ldots, 6$ and we have used the structure constants (A2). Using the map (2.34) we define the corresponding Hermitian matrix

$$
H=\frac{\mathrm{i}}{4}\left(\gamma^{135}+\gamma^{425}+\gamma^{416}+\gamma^{326}\right),
$$

where generally $\gamma^{a_{1} \ldots a_{r}}:=\gamma^{\left[a_{1}\right.} \ldots \gamma^{\left.a_{r}\right]}$. Using the Clifford algebra (5.10) it is straightforward to check that

$H^{2}=\frac{1}{4}\left(\mathbf{1}_{8}-\gamma^{1234}-\gamma^{3456}-\gamma^{1256}\right)$ and $H^{4}=H^{2}$.

Hence $H^{2}$ is a projector, and $\operatorname{Tr}_{8}\left(H^{2}\right)=2$ so it is of rank two. The matrix $H$ itself is traceless with real eigenvalues, so it has six zero eigenvalues and two nonvanishing eigenvalues \pm 1 , each of multiplicity one. The eigenspinors of $H$ are easily determined. Going back to the complex basis (5.9) we can write

$$
H=\mathrm{i}\left(\tilde{\gamma}^{\overline{1} \overline{\overline{3}}}+\tilde{\gamma}^{123}\right) .
$$

Expanding spinors on $\mathbb{F}_{3}$ as in (2.45), one then has

$$
H\left(|\Omega\rangle \pm \mathrm{i} \tilde{\gamma}^{\overline{1} \overline{\overline{2}}}|\Omega\rangle\right)= \pm\left(|\Omega\rangle \pm \mathrm{i} \tilde{\gamma}^{\overline{1} \overline{\overline{2}} \overline{3}}|\Omega\rangle\right)
$$

and

$$
H\left(\tilde{\gamma}^{\bar{\alpha}}|\Omega\rangle\right)=0=H\left(\tilde{\gamma}^{\bar{\alpha} \bar{\beta}}|\Omega\rangle\right) .
$$

The Dirac operator (2.35) for the canonical connection on the nonsymmetric coset space $\mathbb{F}_{3}$ squares to [19]

$$
\begin{aligned}
\left(\mathrm{i} \not D_{q, m}\right)^{2}= & \nabla_{q, m}^{2}+\frac{1}{4} \mathrm{Scal}_{8}-\frac{1}{8} \mathrm{R}_{a b c d} \gamma^{a b c d} \\
& -\frac{\mathrm{i}}{2}\left(f_{q, m}\right)_{a b} \gamma^{a b},
\end{aligned}
$$

where $\nabla_{q, m}^{2}$ is the spinor Laplacian including both the canonical spin connection and the bimonopole gauge connection, and $f_{q, m}$ is the $\mathrm{U}(1) \times \mathrm{U}(1)$ field strength (2.41). The structure constants (A2) and the explicit expression for the Riemann curvature tensor of the canonical connection [19]

$$
\mathrm{R}_{a b c d}=f_{a b 7} f_{c d 7}+f_{a b 8} f_{c d 8}
$$

yield curvature two-forms

$$
\begin{aligned}
& \mathrm{R}_{12}=\frac{1}{6}\left(2 e^{12}-e^{34}-e^{56}\right) \\
& \mathrm{R}_{34}=\frac{1}{6}\left(-e^{12}+2 e^{34}-e^{56}\right) \\
& \mathrm{R}_{56}=\frac{1}{6}\left(-e^{12}-e^{34}+2 e^{56}\right)
\end{aligned}
$$

The Ricci tensor is then $\operatorname{Ric}_{a b}=\frac{1}{3} \delta_{a b}$, the scalar curvature is Scal $=2$, and

$$
\mathrm{R}_{a b c d} \gamma^{a b c d}=-\frac{4}{3}\left(\gamma^{1234}+\gamma^{3456}+\gamma^{1256}\right) .
$$

Combining these expressions we find that the square of the Dirac operator (5.17) can be written as

$$
\left(\mathrm{i} \not D_{q, m}\right)^{2}=\nabla_{q, m}^{2}+\frac{2}{3}\left(\mathbf{1}_{8}-H^{2}\right)-\frac{\mathrm{i}}{2}\left(f_{q, m}\right)_{a b} \gamma^{a b} .
$$

The rank-6 projector $\mathbf{1}_{8}-H^{2}$ is related to the $U(1) \times U(1)$ holonomy group in the following way: the generators of the holonomy group in the spinor representation can be constructed, using the Clifford algebra (5.10), as

$$
\begin{gathered}
T_{7}=-\frac{1}{4} f_{a b 7} \gamma^{a b}=\frac{i}{2 \sqrt{3}}\left(\tilde{\gamma}^{\overline{1}} \gamma^{1}+\tilde{\gamma}^{\overline{2}} \tilde{\gamma}^{2}-2 \tilde{\gamma}^{\overline{3}} \tilde{\gamma}^{3}\right), \\
T_{8}=-\frac{1}{4} f_{a b 8} \gamma^{a b}=-\frac{i}{2}\left(\tilde{\gamma}^{\overline{1}} \gamma^{1}-\tilde{\gamma}^{\overline{2}} \tilde{\gamma}^{2}\right),
\end{gathered}
$$

with

$$
\left[T_{7}, T_{8}\right]=0 .
$$

Then

$$
\frac{1}{3}\left(\mathbf{1}_{8}-H^{2}\right)=-\left(T_{7}^{2}+T_{8}^{2}\right)
$$

is the second order Casimir. It is straightforward to check, using (A16), that $-3\left(I_{7}^{2}+I_{8}^{2}\right)$ is indeed a rank-6 projector in the adjoint representation: the zero eigenvectors are of course the weights $(0,0)_{2}$ and $(0,0)_{0}$. In fact $\left[T_{a}, H\right]=0$, for $a=7,8$.

Let us now look at the zero modes of (5.21), beginning with the case where there are no monopole backgrounds, 
i.e. $(q, m)=(0,0)$ and $\not \not_{0,0}=\not \mathbb{F}_{3}$ is the untwisted canonical Dirac operator from (2.36). By ([25], Thm. 10.8) every harmonic spinor is also parallel for the canonical connection. Now the matrix $\mathbf{1}_{8}-H^{2}$ is a projector of rank six, with a two-dimensional kernel spanned by the Fock space states $\left(\mathbf{1}_{8} \pm \mathrm{i} \tilde{\gamma}^{\overline{1} \overline{2}} \overline{3}\right)|\Omega\rangle$. Since the background flux vanishes, $f_{0,0}=0$, these states generate harmonic modes that comprise two covariantly constant spinors $\chi_{0,0}^{ \pm}$, i.e. $\nabla_{0,0} \chi_{0,0}^{ \pm}=0$. By ([17], Thm. 4.2) a spinor field is parallel with respect to the canonical connection if and only if it is constant, whence there are two constant zero modes with opposite chirality given by

$$
\chi_{0,0}^{+}=|\Omega\rangle \quad \text { and } \quad \chi_{0,0}^{-}=-\tilde{\gamma}^{\overline{1} \overline{2} \overline{3}}|\Omega\rangle .
$$

These states are unique, up to a normalization and a phase, consistent with the index theorem (2.42), which in this case gives $\nu_{0,0}=0$, as then $\operatorname{dim} \operatorname{ker}\left(\not_{0,0}^{+}\right)=\operatorname{dim} \operatorname{ker}\left(\not \not_{0,0}^{-}\right)=$ 1 . The states (5.26) correspond to the doubly degenerate weight $(0,0)_{0,2}$ of the adjoint representation, as discussed in Sec. III B; these states are represented by the red nodes in the $(k, l)=(1,1)$ diagram of Fig. 3. They form the components of the canonical covariantly constant spinor $\chi_{0,0}=$ $\chi_{0,0}^{+} \oplus \chi_{0,0}^{-}$associated with the family of SU(3) structures on $\mathbb{F}_{3}$ at the nearly Kähler point (2.29) of the moduli space.

For $(q, m) \neq(0,0)$, the constant spinors (5.26) are no longer zero modes, because of both the inhomogeneous field strength term in (5.21) and the bimonopole connection featuring in the Dirac Laplacian $\nabla_{q, m}^{2}$. However, by the above analysis there are also six constant eigenspinors of $i \not \not_{\mathbb{F}_{3}}^{1}$ with eigenvalues $\pm \sqrt{2 / 3}$ each of multiplicity three from (5.16). Moreover, from (2.41), (2.25), and (2.26) we find

$\left(f_{q, m}\right)_{a b} \gamma^{a b}=-\frac{\mathrm{i}}{24}\left((m-q) \tilde{\gamma}^{1 \overline{1}}-(m+q) \tilde{\gamma}^{2 \overline{2}}+2 q \tilde{\gamma}^{3 \overline{3}}\right)$.

Hence by choosing the background bimonopole charges $(q, m)$ appropriately we can cancel both the flux $\frac{2}{3}\left(\mathbf{1}_{8}-H^{2}\right)$ in (5.21) and the corresponding contribution to (2.35) of the canonical connection; note that this cancellation relies crucially on the fact that the canonical connection (2.23) is valued in the Cartan subalgebra $u(1) \oplus u(1)$, like the background bimonopole gauge potentials. From (2.23) it follows that these charges are precisely the nonzero weights of the adjoint representation, and for each such charge there is a unique constant harmonic spinor $\chi_{\bar{q}, m}^{ \pm}$; these states are represented by the blue nodes in the $(k, l)=(1,1)$ diagram of Fig. 3. Each of these charges will turn one of the six constant but nonzero modes of the untwisted Dirac operator i $\not \phi_{\mathbb{F}_{3}}^{1}$ into a constant zero mode, while at the same time turning the two constant zero modes of $i \not_{\mathbb{F}_{3}}^{1}$ into constant nonzero modes. From the $\mathrm{U}(1) \times \mathrm{U}(1)$ charge assignments given by (3.25) and (3.26), the three positive chirality zero modes are $\chi_{-2,0}^{+}=\tilde{\gamma}^{\overline{1} \overline{2}}|\Omega\rangle, \quad \chi_{1,3}^{+}=\tilde{\gamma}^{\overline{3}} \overline{1}|\Omega\rangle \quad$ and $\quad \chi_{1,-3}^{+}=\tilde{\gamma}^{\overline{2} \overline{3}}|\Omega\rangle$,

while the three negative chirality zero modes are

$\chi_{-1,3}^{-}=\tilde{\gamma}^{\overline{1}}|\Omega\rangle, \quad \chi_{-1,-3}^{-}=\tilde{\gamma}^{\overline{2}}|\Omega\rangle \quad$ and $\quad \chi_{2,0}^{-}=\tilde{\gamma}^{\overline{3}}|\Omega\rangle$.

In fact, these are the only bimonopole fields that give rise to twisted spinor bundles that admit unique, constant chiral harmonic modes: For a given representation $\hat{V}^{k, l}$, it is straightforward to show from the index formula (2.42) that there are only six possible weights $(q, m)_{n} \in W^{k, l}$ yielding index $\nu_{q, m}= \pm 1$, given as

$(q, m)=(1,3), \quad(-2,0), \quad(1,-3) \quad$ with $\quad \nu_{q, m}=+1$

and

$(q, m)=(-1,-3), \quad(2,0), \quad(-1,3) \quad$ with $\quad \nu_{q, m}=-1$,

consistent with the $\mathrm{U}(1) \times \mathrm{U}(1)$ charge assignments in (5.28) and (5.29).

Returning to the zero modes (5.26) we shall now show that they are actually linear combinations of $S U(3)$ weights. The $U(1) \times U(1)$ action (5.22) and (5.23) on spinors can be extended to $S U(2) \times U(1)$ by constructing two more generators,

$$
T_{5}=-\frac{1}{4} f_{a b 5} \gamma^{a b}, \quad T_{6}=-\frac{1}{4} f_{a b 6} \gamma^{a b},
$$

where $\gamma^{7}:=i \gamma^{123456}=\tilde{\gamma}$ is the chirality operator in (2.38). ${ }^{1}$ Raising and lowering operators can now be built,

$$
\begin{aligned}
& T_{+}=\frac{1}{2}\left(T_{5}+i T_{6}\right)=\frac{1}{2 \sqrt{3}}\left(\tilde{\gamma}^{\overline{1} \overline{2}}-i \tilde{\gamma}^{3} \tilde{\gamma}\right), \\
& T_{-}=\frac{1}{2}\left(T_{5}-i T_{6}\right)=\frac{1}{2 \sqrt{3}}\left(\tilde{\gamma}^{12}+i \tilde{\gamma}^{\overline{3}} \tilde{\gamma}\right),
\end{aligned}
$$

which, together with $T_{7}$ in Eq. (5.22), generate $S U(2)$,

$\left[T_{+}, T_{-}\right]=\frac{1}{2 \sqrt{3}} T_{7}, \quad\left[T_{7}, T_{ \pm}\right]= \pm \frac{i}{2 \sqrt{3}} T_{ \pm}$.

Equations (5.33) and (5.34) give, with (5.28) and (5.29),

$T_{+} \chi_{2,0}^{-}=\frac{i}{2 \sqrt{3}}\left(\left|\Omega>-i \tilde{\gamma}^{\overline{1} \overline{2} \overline{3}}\right| \Omega>\right)=\frac{i}{2 \sqrt{3}}\left(\chi_{0,0}^{+}-i \chi_{0,0}^{-}\right)$,

\footnotetext{
${ }^{1}$ There is no $\gamma^{8}$ in this construction, but it works because, with the structure constants in (A2), no $\gamma^{8}$ ever appears on the righthand sides of (5.32).
} 


$$
\begin{aligned}
T_{-} \chi_{-2,0}^{+} & =-\frac{1}{2 \sqrt{3}}\left(\left|\Omega>-i \tilde{\gamma}^{\overline{1} \overline{\overline{2}} \overline{3}}\right| \Omega>\right) \\
& =-\frac{1}{2 \sqrt{3}}\left(\chi_{0,0}^{+}-i \chi_{0,0}^{-}\right) .
\end{aligned}
$$

Thus $\chi_{2,0}^{-}, \frac{1}{\sqrt{2}}\left(\chi_{0,0}^{+}-i \chi_{0,0}^{-}\right)$and $\chi_{2,0}^{-}$form a natural $S U(2)$ triplet, and we can identify, up to inconsequential phases,

$$
\chi_{(0,0)_{2}}=\frac{1}{\sqrt{2}}\left(\chi_{0,0}^{+}-i \chi_{0,0}^{-}\right) .
$$

The orthogonal combination

$$
\chi_{(0,0)_{0}}=\frac{1}{\sqrt{2}}\left(-i \chi_{0,0}^{+}+\chi_{0,0}^{-}\right)
$$

is an $S U(2)$ singlet annihilated by $T_{ \pm} \cdot \chi_{(0,0)_{2}}$ and $\chi_{(0,0)_{0}}$ are in fact the eigenspinors of $H$ in (5.15). Thus the two zero modes associated with the weights $(0,0)_{2}$ and $(0,0)_{0}$ are not themselves Weyl spinors; they are Dirac spinors that are linear combinations of two Weyl spinors.

It is possible to classify completely all representations $\hat{V}^{k, l}$ that give rise to such spinor harmonics. For a given irreducible representation of SU(3), one can work out the index for each weight using (2.42) (see Fig. 3); note that for triangular quivers $Q^{k, 0}$ the index (2.42) can be parametrized using (A15) as

$$
\nu_{k ; q, n}=\frac{1}{8} q\left(q^{2}-(2 k-3 n)^{2}\right),
$$

where $n=0,1, \ldots, k$ labels the hypercharge levels and $q \in\{-n,-(n-2), \ldots, n-2, n\}$. The smallest irreducible representation in which the weights (5.30) and (5.31) appear is the adjoint representation of $\mathrm{SU}(3)$ with $(k, l)=$ $(1,1)$, while the next one is the decuplet representation with $(k, l)=(3,0)$; in both cases these six possibilities, together with the nodes $(0,0)$ at the center, represent the only zero modes that arise. They can also occur in higher irreducible representations $\hat{V}^{k, l}$, but only in those with weight diagrams $W^{k, l}$ that have the $\mathbb{Z}_{3}$ symmetry that allows them to contain the outer hexagonal layer of the adjoint diagram $W^{1,1}$ (Fig. 3). From the explicit construction of the weights $(q, m)_{n}$ given in (A15), it is straightforward to see that the only weight diagrams $W^{k, l}$ that contain the weights (5.30) and (5.31) correspond to representations

$$
\hat{V}^{k, l} \quad \text { with } \quad k-l \equiv 0 \bmod 3 .
$$

By (5.30) and (5.31), each of the blue nodes in Fig. 3 corresponds to a twisted spinor bundle over $\mathbb{F}_{3}$, which admits a unique constant zero mode of either positive or negative chirality.

For a generic representation in the class (5.41) with $k \geq$ $l$, the bottom edge of the adjoint hexagon has nodes with hypercharge $m=-3$. Recall from our analysis of the combinatorics of weight diagrams from Sec. IV D that the inverted triangular layer of a weight diagram $W^{k, l}$ starts at level $m=-2(k-l)$ (see Fig. 2). Hence for $k>l$ the adjoint hexagon lies inside the triangle and each of its weight states therefore has multiplicity $l+1$, while for $k=l$ there are no triangular layers and the states have multiplicity $l$; in both cases the node $(0,0)_{n}$ in the center of the hexagon has degeneracy $l+1$. For a fixed weight $(q, m)_{n}$ in the adjoint hexagon, from (A15) it follows that the isospin labels can be parametrized as $n=n_{j}$ where

$n_{j}=2 j+\frac{2}{3}(k-l)+\frac{m}{3} \quad$ with $\quad j:=2 j_{-}=0,1, \ldots, l$

for $k>l$, while for $k=l$ we have $j \neq 0$ when $m=-3$ or $m=0, q= \pm 2$ and $j \neq l$ for $m=3$.

If we normalize the fermionic Fock vacuum $|\Omega\rangle$ such that

$$
\langle\Omega \mid \Omega\rangle=\operatorname{Vol}\left(\mathbb{F}_{3}\right)^{-1},
$$

then the nonzero Yukawa couplings around the adjoint hexagon are denoted by

$$
\begin{aligned}
& \tilde{x}_{j}^{+}:=-\tilde{Y}_{-1 ; 1,1}^{(2,0)_{n_{j}},(1,3)_{n_{j}-1}}=\sqrt{\frac{n_{j}+2}{2\left(n_{j}+1\right)}} \lambda_{k, l}^{-}\left(n_{j}, 0\right), \\
& \tilde{x}_{j}^{-}:=\tilde{Y}_{-1 ; 1,1}^{(-1,-3)_{n_{j}},(-2,0)_{n_{j}+1}}=\sqrt{\frac{n_{j}+3}{2\left(n_{j}+1\right)}} \lambda_{k, l}^{+}\left(n_{j},-3\right), \\
& \tilde{y}_{j}^{+}:=\tilde{Y}_{-2 ; 1,1}^{(2,0)_{n_{j}},(1,-3)_{n_{j}-1}}=\sqrt{\frac{n_{j}+2}{2 n_{j}} \lambda_{k, l}^{+}\left(n_{j}-1,-3\right),} \\
& \tilde{y}_{j}^{-}:=-\tilde{Y}_{-2 ; 1,1}^{(-1,3)_{n_{j}},(-2,0)_{n_{j}+1}}=\sqrt{\frac{n_{j}+3}{2\left(n_{j}+2\right)}} \lambda_{k, l}^{-}\left(n_{j}+1,0\right)
\end{aligned}
$$

for the diagonal links, and

$\tilde{z}_{j}^{0}:=\tilde{Y}_{-3 ; 1,1}^{(-1,3)_{n_{j}},(1,3)_{n_{j}}}=-\tilde{Y}_{-3 ; 1,1}^{(-1,-3)_{n_{j}},(1,-3)_{n_{j}}}=\frac{n_{j}+1}{2}$

for the horizontal links [all the relevant fermion bilinears in (5.5) evaluate to \pm 1 for the constant spinor harmonics (5.28) and (5.29)].

To evaluate the nonvanishing Yukawa couplings corresponding to arrows associated with the center nodes $(0,0)_{n_{j}}$, we must allow for possible mixing between the $S U(3)$ weights and the chiral zero modes. Let the central $S U(3)$ weights be $(0,0)_{n_{>}}$and $(0,0)_{n_{<}}$, with $n_{>}=n_{<}+2$, and then the corresponding zero modes can be taken to be $\chi_{(0,0)_{n>}}$ and $\chi_{(0,0)_{n<}}$, where

$$
\begin{aligned}
& \chi_{(0,0)_{n_{<}}}=\left(u_{n_{<}} \chi_{0,0}^{+}+v_{n_{<}} \chi_{0,0}^{-}\right), \\
& \chi_{(0,0)_{n>}}=\left(u_{n_{>}} \chi_{0,0}^{+}+v_{n_{>}} \chi_{0,0}^{-}\right)
\end{aligned}
$$

is a unitary transformation. We adopt a slight modification in notation for the central weights $(0,0)_{n_{>}}$and $(0,0)_{n_{<}}$of the adjoint under consideration here: because $\chi_{(0,0)_{n>}}$ and $\chi_{(0,0)_{n}}$ are not in general chiral, the two associated spinor 
fields on $M$ will be denoted $\rho_{(0,0)_{n>}}$ and $\rho_{(0,0)_{n_{<}}}$, and the expansion of $\Psi_{0}$ in (3.18) becomes

$$
\Psi_{0}=\rho_{(0,0)_{n_{>}}} \otimes \chi_{(0,0)_{n>}}+\rho_{(0,0)_{n_{<}}} \otimes \chi_{(0,0)_{n_{<}}} .
$$

The relevant Fermion bilinears then evaluate to give Yukawa couplings

$$
\begin{aligned}
& \tilde{\xi}_{j}^{ \pm}:=\tilde{Y}_{+1 ; 1,1}^{(0,0)_{n_{j}},(-1,3)_{n_{j} \mp 1}}=\sqrt{\frac{n_{j} \mp 1+1}{2\left(n_{j}+1\right)}} \lambda_{k, l}^{\mp}\left(n_{j}, 0\right) u_{n_{j}}^{*}, \\
& \tilde{\xi}_{j}^{\prime \pm}:=\tilde{Y}_{+1 ; 1,1}^{(1,-3)_{n_{j}},(0,0)_{n_{j} \mp 1}}=\frac{1}{\sqrt{2}} \lambda_{k, l}^{\mp}\left(n_{j},-3\right) v_{n_{j} \mp 1}, \\
& \tilde{\zeta}_{j}^{ \pm}:=\tilde{Y}_{+2 ; 1,1}^{(0,0)_{n_{j},},(-1,-3)_{n_{j} \mp 1}}=\frac{1}{\sqrt{2}} \lambda_{k, l}^{ \pm}\left(n_{j} \mp 1,-3\right) u_{n_{j}}^{*}, \\
& \tilde{\zeta}_{j}^{\prime \pm}:=\tilde{Y}_{+2 ; 1,1}^{(1,3)_{n_{j}},(0,0)_{n_{j} \mp 1}}=\sqrt{\frac{n_{j}+1}{2\left(n_{j} \mp 1+1\right)}} \lambda_{k, l}^{ \pm}\left(n_{j} \mp 1,0\right) v_{n_{j} \mp 1}
\end{aligned}
$$

for the diagonal links, while

$$
\tilde{\zeta}_{j}^{0}:=\tilde{Y}_{+3 ; 1,1}^{(0,0)_{n_{j}},(2,0)_{n_{j}}}=\frac{1}{2} \sqrt{n_{j}\left(n_{j}+2\right)} u_{n_{j}}^{*},
$$

$$
\tilde{\zeta}_{j}^{\prime 0}:=\tilde{Y}_{+3 ; 1,1}^{(-2,0)_{n_{j}},(0,0)_{n_{j}}}=\frac{1}{2} \sqrt{n_{j}\left(n_{j}+2\right)} v_{n_{j}}
$$

for the horizontal links, where the \pm superscripts on the left-hand side of (5.49) are both included in the $k=l$ cases to account for the doubling of arrows between layers of different weight multiplicities, and we have utilized the combinatorial counting of physical fields from Sec. IV D.

In the following we abbreviate $\psi_{(q, m)_{n}}:=\psi_{(q, m)_{n} ; 1}$, $\breve{\psi}_{(q, m)_{n}}:=\breve{\psi}_{(q, m)_{n} ; 1}=\gamma \eta_{(q, m) ; 1}$ and $\breve{\rho}_{(q, m)_{n}}:=\gamma \rho_{(q, m)_{n}}$. Then the constant spinor contributions to the Yukawa interaction Lagrangian (5.7), including the contribution from $\Psi^{0}$ in (3.17), read as

$$
\begin{aligned}
& \mathcal{L}_{M}^{\mathrm{Y} 0}=2 i g \sum_{j}\left(-\tilde{x}_{j}^{+} \breve{\psi}_{(2,0)_{n_{j}}}{ }^{\dagger} \wedge \star \phi_{(2,0)_{n_{j}}}^{1}{ }^{(-) / \dagger} \psi_{(1,3)_{n_{j}-1}}+\tilde{x}_{j}^{-} \breve{\psi}_{(-1,-3)_{n_{j}}}{ }^{\dagger} \wedge \star \phi_{(-1,-3)_{n_{j}}}^{1}{ }^{(+) / \dagger} \psi_{(-2,0)_{n_{j}+1}}+\tilde{y}_{j}^{+} \breve{\psi}_{(2,0)_{n_{j}}}{ }^{\dagger}\right. \\
& \wedge \star \phi_{(2,0)_{n_{j}}}^{2}{ }^{(-) / \dagger} \psi_{(1,-3)_{n_{j}-1}}-\tilde{y}_{j}^{-} \breve{\psi}_{(-1,3)_{n_{j}}}{ }^{\dagger} \wedge \star \phi_{(-1,3)_{n_{j}}}^{2}{ }^{(+) / \dagger} \psi_{(-2,0)_{n_{j}+1}}+\tilde{z}_{j}^{0}\left(-\breve{\psi}_{(-1,-3)_{n_{j}}}{ }^{\dagger} \wedge \star \phi_{(-1,-3)_{n_{j}}}{ }^{\dagger} \psi_{(1,-3)_{n_{j}}}\right. \\
& +\breve{\psi}_{(-1,3)_{n_{j}}}^{\dagger} \wedge \star \phi_{(-1,3)_{n_{j}}}^{3 \prime} \psi_{(1,3)_{n_{j}}}{ }^{\dagger}+\tilde{\xi}_{j}^{\mp} \rho_{(0,0)_{n_{j}}}{ }^{\dagger} \wedge \star \phi_{(0,0)_{n_{j}}}^{1}{ }^{( \pm) / \dagger} \breve{\psi}_{(-1,3)_{n_{j} \pm 1}}+\tilde{\xi}_{j}^{\prime \mp} \psi_{(1,-3)_{n_{j}}}{ }^{\dagger} \wedge \star \phi_{(1,-3)_{n_{j}}}{ }^{( \pm) / \dagger} \breve{\rho}_{(0,0)_{n_{j} \pm 1}} \\
& +\tilde{\zeta}_{j}^{\mp} \rho_{(0,0)_{n_{j}}}{ }^{\dagger} \wedge \star \phi_{(0,0)_{n_{j}}}^{2}{ }^{( \pm) / \dagger} \breve{\psi}_{(-1,-3)_{n_{j} \pm 1}}+\tilde{\zeta}_{j}^{\prime \mp} \psi_{(1,3)_{n_{j}}}{ }^{\dagger} \wedge \star \phi_{(1,3)_{n_{j}}}^{2}{ }^{( \pm) / \dagger} \breve{\rho}_{(0,0)_{n_{j} \pm 1}}+\tilde{\zeta}_{j}^{0} \psi_{(0,0)_{n_{j}}}{ }^{\dagger} \wedge \star \phi_{(0,0)_{n_{j}}}^{3 l}{ }^{\dagger} \breve{\psi}_{(2,0)_{n_{j}}} \\
& \left.+\zeta^{10} \psi_{(-2,0)_{n_{j}}}^{\dagger} \wedge \star \phi_{(-2,0)_{n_{j}}}^{3 \prime} \breve{\rho}_{(0,0)_{n_{j}}}\right) \text {, }
\end{aligned}
$$

together with its Hermitian conjugate, with an implicit sum over \pm for the real representations with $k=l$. Thus the quiver gauge theory contains Yukawa interactions for every quiver derived from an $\mathrm{SU}(3)$ representation of the form (5.41). If the Higgs fields appearing in (5.52) acquire a nonzero vacuum expectation value through dynamical symmetry breaking, then the $8(l+1)-6 \delta_{k l}$ fermion fields occurring in (5.52) acquire a mass matrix. In the special case
(4.38), the eigenvalues of this mass matrix, like the perturbative induced gauge and Higgs boson masses, are independent of the gauge coupling $g$ and determined entirely by the metric moduli $\boldsymbol{s}_{\alpha}$ of the coset space $\mathbb{F}_{3}$.

As an explicit example, let us consider the simplest nontrivial case of the adjoint representation with $(k, l)=$ $(1,1)$. The Yukawa couplings in (5.52) can be associated with the arrows in the quiver diagram

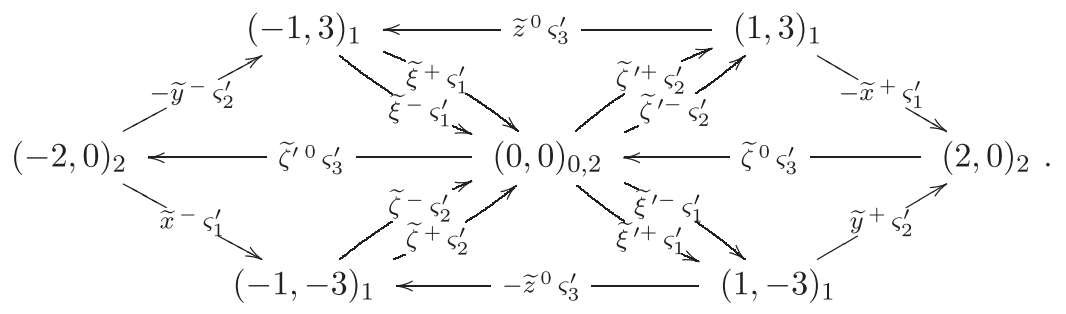

Using (A18), together with the values of $u_{2}, u_{0}, v_{2}$ and $v_{0}$ from (5.38) and (5.39), the Yukawa coupling coefficients (5.44), (5.45), (5.49), (5.50), and (5.51) can be computed explicitly, and after substituting the Higgs vacuum (4.38) with $V_{(q, m)_{n}}^{1( \pm)}=V_{(q, m)_{n}}^{2}=V_{(q, m)_{n}}^{3( \pm)}=\mathbf{1}_{p}$ the induced $8 \times 8$ fermion mass matrix $\boldsymbol{\mu}$ can be read off from (5.52).
Then, with the relevant symmetric spinors organized into an eight-dimensional vector,

$$
\begin{aligned}
\boldsymbol{\psi}= & \left(\eta_{(2,0)_{2}}, \rho_{(0,0)_{2}}, \psi_{(-2,0)_{2}}, \psi_{(1,3)_{1}}, \eta_{(-1,3)_{1}}, \eta_{(-1,-3)_{1}},\right. \\
& \left.\psi_{(1,-3)_{1}}, \rho_{(0,0)_{0}}\right)^{T},
\end{aligned}
$$

the mass matrix in $\boldsymbol{\psi}^{\dagger} \gamma \boldsymbol{\mu} \boldsymbol{\psi}$ is 


$$
\boldsymbol{\mu}=2 i g\left(\begin{array}{cccccccc}
0 & -\varsigma_{3} & 0 & -\varsigma_{1} & 0 & 0 & \varsigma_{2} & 0 \\
\varsigma_{3} & 0 & -i \varsigma_{3} & -i \frac{\varsigma_{2}}{2} & \frac{\varsigma_{1}}{2} & \frac{\varsigma_{2}}{2} & -i \frac{\varsigma_{1}}{2} & 0 \\
0 & -i \varsigma_{3} & 0 & 0 & \varsigma_{2} & -\varsigma_{1} & 0 & 0 \\
\varsigma_{1} & -i \frac{\varsigma_{2}}{2} & 0 & 0 & -\varsigma_{3} & 0 & 0 & \frac{\sqrt{3}}{2} \varsigma_{2} \\
0 & -\frac{\varsigma_{1}}{2} & -\varsigma_{2} & \varsigma_{3} & 0 & 0 & 0 & i \frac{\sqrt{3}}{2} \varsigma_{1} \\
0 & -\frac{\varsigma_{2}}{2} & \varsigma_{1} & 0 & 0 & 0 & -\varsigma_{3} & i \frac{\sqrt{3}}{2} \varsigma_{2} \\
-\varsigma_{2} & -i \frac{\varsigma_{1}}{2} & 0 & 0 & 0 & \varsigma_{3} & 0 & \frac{\sqrt{3}}{2} \varsigma_{1} \\
0 & 0 & 0 & -\frac{\sqrt{3}}{2} \varsigma_{2} & i \frac{\sqrt{3}}{2} \varsigma_{1} & i \frac{\sqrt{3}}{2} \varsigma_{2} & -\frac{\sqrt{3}}{2} \varsigma_{1} & 0
\end{array}\right)
$$

together with the constraint $\varsigma_{1} \varsigma_{2} \varsigma_{3}=\frac{\sqrt{3}}{72}$. The matrix (5.55) is Hermitian, it can be diagonalized and its eigenvalues can be determined as functions of the metric moduli $\varsigma_{\alpha}, \alpha=1$, 2,3 ; the mass eigenvalues come in charge conjugate pairs $\pm \mu_{i}, i=1, \ldots, 4$. The explicit expression for the eigenvalues are not illuminating for generic $\boldsymbol{s}_{\alpha}$, and here we just note that the determinant of the mass matrix is given by

$$
192 g^{8}\left(\varsigma_{2}^{2}+\varsigma_{1}^{2}\right)^{2}\left(\varsigma_{2}^{2}+\varsigma_{1}^{2}+2 \varsigma_{3}^{2}\right)^{2},
$$

so there are never any massless fermions in this sector of the spectrum on $M$.

We should stress that this analysis does not necessarily classify all possible Yukawa interactions, as we have not precluded the possibility that the coupling coefficients (5.5) and (5.6) may be nonvanishing for fermion bilinears associated with pairings between weight states $(q, m)_{n} \in$ $W^{k, l}$ associated with indices of equal magnitude $\left|\nu_{q, m}\right|>1$ and opposite sign (see Fig. 3); however, we have shown that such harmonic spinors are necessarily nonconstant, and the evaluation of the integrals in (5.5) and (5.6) requires their explicit construction, which we will not attempt here. Nevertheless, we have classified all couplings associated with constant harmonic spinors, equivalently symmetric fermions corresponding to unique Dirac zero modes, and found a large class of quasi-Kähler SU(3) structures, including the nearly Kähler point, which admit chirally paired fermion mass generation.

\section{Symmetric spinors of torsion class $W_{1} \oplus W_{2}$}

It is interesting to study how the Yukawa couplings vary as we move around the moduli space. Unfortunately, the situation is far less under control for generic values of the metric moduli $\varsigma_{\alpha}$, as the constant spinor fields $\chi$ on $\mathbb{F}_{3}$ are no longer zero modes of the untwisted Dirac operator $\not_{\mathbb{F}_{3}}^{\sigma}$. By ([17], Thm. 4.2), constant spinors are eigenspinors of the square $\left(i \not \partial_{\mathbb{F}_{3}}^{\sigma}\right)^{2}$ with the eigenvalue equation

$$
\left(\mathrm{i} \not \not_{\mathbb{F}_{3}}^{\sigma}\right)^{2} \chi=\frac{9}{4}(1-\sigma)^{2} \chi \quad \text { for } \chi=\text { const. }
$$

Hence in the generic case the adjoint octet of constant spinors (5.26), (5.28), and (5.29) play no role in the construction of symmetric fermions, and an explicit determination of the Yukawa coupling coefficients (5.5) requires a more detailed understanding of the nonconstant spinor harmonics. The Yukawa couplings in this case come from weight states $(q, m)_{n} \in W^{k, l}$ corresponding to higherdimensional Dirac kernels $\operatorname{ker}\left(\not \not_{q, m}^{ \pm}\right)$, and because they can only arise on links between nodes of the same index $\pm \nu_{q, m}$, we expect that for a given SU(3) module $\hat{V}^{k, l}$ there will be far fewer Yukawa interactions between symmetric fermions, if any (see Fig. 3). One might regard this feature as a further physical vindication for specifically constraining the heterotic flux compactification to the nearly Kähler locus of the moduli space, a requirement that usually follows from supersymmetry considerations [6].

A representative class of quasi-Kähler SU(3) structures that does not include the nearly Kähler point $\boldsymbol{s}_{\alpha}=\frac{1}{2 \sqrt{3}}$, $\alpha=1,2,3$ occurs on the surface $\sigma=\frac{1}{3}$ in the moduli space. Recall that this is the only other known case where the index $\left|\nu_{q, m}\right|$ corresponds to the dimension of the vector space $\operatorname{ker}\left(\not \not_{q, m}\right)$ of harmonic spinors. Moreover, in this case any constant spinor is a zero mode of the operator $\left(i \not \not_{\mathbb{F}_{3}}^{1 / 3}\right)^{2}-\mathbf{1}_{8}$. The complexity of the change in structure of the harmonic spinors in this case is further exemplified by noting that the analog of the relation (5.21) for $\sigma=\frac{1}{3}$ is given by [18,21]

$$
\begin{aligned}
\left(\mathrm{i} \not D_{q, m}\right)^{2}= & \nabla_{q, m}^{2}-\frac{2}{3} H^{2}+\frac{1}{2}\left(\mathbf{1}_{8}+K_{a b c d} \gamma^{a b c d}\right) \\
& -\frac{\mathrm{i}}{2}\left(f_{q, m}\right)_{a b} \gamma^{a b} .
\end{aligned}
$$

The torsional curvature $K:=\mathrm{d} H=\sqrt{3} \operatorname{Im~} \mathrm{d} \Omega$ can be computed by using the Cartan structure equations for the frame $\tilde{\Theta}^{\alpha}$ from Sec. II B to get

$$
\mathrm{d} \Omega=W_{1} \tilde{\omega} \wedge \tilde{\omega}+W_{2} \wedge \tilde{\omega}
$$

where

$$
W_{1}=\frac{1}{36}\left(\frac{1}{\varsigma_{1}^{2}}+\frac{1}{\varsigma_{2}^{2}}+\frac{1}{\varsigma_{3}^{2}}\right)
$$

and 


$$
\begin{aligned}
W_{2}= & \frac{\mathrm{i}}{432}\left(\left(\frac{2}{\varsigma_{1}^{2}}-\frac{1}{\varsigma_{2}^{2}}-\frac{1}{\varsigma_{3}^{2}}\right) \tilde{\Theta}^{1} \wedge \tilde{\Theta}^{\overline{1}}-\left(\frac{2}{\varsigma_{2}^{2}}-\frac{1}{\varsigma_{1}^{2}}-\frac{1}{\varsigma_{3}^{2}}\right) \tilde{\Theta}^{2}\right. \\
& \left.\wedge \tilde{\Theta}^{\overline{2}}+\left(\frac{2}{\varsigma_{3}^{2}}-\frac{1}{\varsigma_{1}^{2}}-\frac{1}{\varsigma_{2}^{2}}\right) \tilde{\Theta}^{3} \wedge \tilde{\Theta}^{\overline{3}}\right)
\end{aligned}
$$

together with the constraint $\varsigma_{1} \varsigma_{2} \varsigma_{3}=\frac{\sqrt{3}}{72}$. While the canonical connection $\Gamma$ at $\sigma=1$ on $\mathbb{F}_{3}$ appears in the supersymmetry condition that demands that the supersymmetry parameter be a covariantly constant spinor with respect to it, and hence equal to $\chi_{0,0}$ from (5.26), the two-parameter family of connections $\tilde{\Gamma}$ at $\sigma=\frac{1}{3}$ is the one that appears in the Dirac zero mode equation for the $d$-dimensional gaugino field on $M$.

\section{Symmetric spinors of torsion class $W_{5}$}

Finally, let us consider the Yukawa couplings for the standard Kähler geometry of the homogeneous space $\mathbb{F}_{3}$. The relevant connection in this case is the Levi-Cività connection $\hat{\Gamma}$ from (2.19) at the locus (2.20), and hence it formally corresponds to the $\sigma=0$ member of the family of Dirac operators (2.36) on $\mathbb{F}_{3}$. Hence the same remarks concerning the $\sigma=\frac{1}{3}$ case of Sec. V C apply here as well, but with two further complications. First, there is no nice simplification for the square of the Dirac operator $\left(\mathrm{i} \not \not_{q, m}\right)^{2}$ in this case, such as that in (5.21) for $\sigma=1$ and that of (5.58) for $\sigma=\frac{1}{3}$; the rather cumbersome formula can be found in ([17], Thm. 3.2). The issue here is that $\mathbb{F}_{3}$ is a nonsymmetric coset space, and moreover the Levi-Cività connection (2.19) is valued in the Lie algebra $\mathrm{SU}(3)$ so there is no way to cancel its off-diagonal components using solely the background bimonopole fields that take values in the Cartan subalgebra $\mathrm{u}(1) \oplus \mathrm{u}(1)$; as we saw explicitly in Sec. VB, the presence of torsion gets around the Lichnerowicz theorem, which would otherwise forbid the construction of harmonic spinors as parallel spinors. Second, strictly speaking, the Kähler structure on $\mathbb{F}_{3}$ does not really live in the family of quasi-Kähler $\mathrm{SU}(3)$ structures parametrized by the metric moduli $\mathrm{s}_{\alpha}, \alpha=1,2,3$; passing to the Kähler locus corresponds to a discontinuous change of complex structure $\mathcal{J}_{+} \leftrightarrow \mathcal{J}_{-}$on $T^{*} \mathbb{F}_{3}$, which cannot be implemented by smoothly varying any continuous parameters like $\sigma$ or $\mathrm{s}_{\alpha}$.

Let us briefly spell out how discrete changes in the complex structure on $\mathbb{F}_{3}$ induce discrete changes in the Yukawa couplings. For the almost Kähler structure on $\mathbb{F}_{3}$ given by (2.17), suitable gamma matrices on $M \times \mathbb{F}_{3}$ are constructed as in (3.20) but now using gamma matrices $\hat{\gamma}^{\alpha}$, $\hat{\gamma}^{\bar{\alpha}}$ with complex orthonormal indices $\alpha=1,2,3$ with respect to the metric $\hat{\mathrm{g}}$. We then use (3.9) with $\tilde{\Theta}^{\alpha}=$ $\frac{\Lambda}{\sqrt{3}} \Theta^{\alpha}=\frac{\Lambda}{\sqrt{3}} \hat{\theta}^{\alpha}$ for $\alpha=1,2$ and $\tilde{\Theta}^{3}=\frac{R}{\sqrt{3}} \Theta^{3}=\frac{R}{\sqrt{3}} \hat{\theta}^{\overline{3}}$, together with the obvious modification of the Clifford map (2.34), to replace the orthonormal one-forms $\hat{\theta}^{\alpha}$ by $\hat{\Gamma}^{\alpha}=\gamma \otimes \hat{\gamma}^{\alpha}$. Note that the discrete change of complex structure
$\mathcal{J}_{+} \leftrightarrow \mathcal{J}_{-}$sends $\tilde{\gamma}^{3} \leftrightarrow \hat{\gamma}^{\overline{3}}$ and hence changes the Yukawa couplings, and also $I_{3}^{-} \leftrightarrow I_{\overline{3}}^{+}=-\left(I_{3}^{-}\right)^{\dagger}$, and so it further changes the group theory coefficients determining the dynamical fermion masses. The $d$-dimensional Yukawa interactions on $M$ are again of the form (5.7) but now with the set of Yukawa coupling coefficients (5.5) replaced by

$$
\begin{aligned}
\hat{Y}_{+1 ; \ell, \ell^{\prime}}^{(q, m)_{n},(q-1, m+3)_{n \pm 1}}= & \Lambda \sqrt{\frac{n \mp q+1 \pm 1}{6(n+1)}} \lambda_{k, l}^{ \pm}(n, m) \\
& \times \int_{\mathbb{F}_{3} \frac{\hat{\omega}^{\wedge 3}}{3 !} \chi_{q, m ; \ell}^{+} \dagger^{\dagger}} \hat{\gamma}^{1} \chi_{q-1, m+3 ; \ell^{\prime}}^{-} \\
\hat{Y}_{+2 ; \ell, \ell^{\prime}}^{(q, m)_{n},(q-1, m-3)_{n \pm 1}}= & \Lambda \sqrt{\frac{n \mp q+1 \pm 1}{6(n+1 \pm 1)}} \lambda_{k, l}^{\mp}(n, m) \\
& \times \int_{\mathbb{F}_{3} \frac{\hat{\omega}^{\wedge 3}}{3 !} \chi_{q, m ; \ell}^{+} \hat{\gamma}^{\dagger} \hat{\gamma}^{2} \chi_{q-1, m-3 ; \ell^{\prime}}^{-},} \\
\hat{Y}_{+3 ; \ell, \ell^{\prime}}^{(q, m)_{n}(q+2, m)_{n}}= & R \sqrt{\frac{(n-q)(n+q+2)}{12}} \\
& \times \int_{\mathbb{F}_{3} \frac{\hat{\omega}^{\wedge 3}}{3 !} \chi_{q, m ; \ell}^{+} \hat{\gamma}^{\dagger} \chi_{q+2, m ; \ell^{\prime}}^{-},}
\end{aligned}
$$

and similarly for (5.6).

It is probable that some Yukawa couplings that are zero in the quasi-Kähler case will become nonzero in this case, and vice versa. Again, without an explicit construction of the nonconstant spinor zero modes on $\mathbb{F}_{3}$, it is not possible to be more specific, but the important point here is that the choice of almost Kähler structure on the internal coset space influences the Yukawa interactions in the dimensionally reduced field theory. Whether the choice of the nearly Kähler structure described in Sec. V B leads to phenomenologically more viable heterotic string vacua will require further detailed investigation.

\section{CONCLUSIONS}

A detailed study has been carried out of the equivariant dimensional reduction of Yang-Mills-Dirac theory over the space $M \times \mathbb{F}_{3}$, with particular attention paid to the Higgs and Yukawa sectors of the resulting field theory on $M$. The study is motivated by heterotic string theory, and although our model lacks two features of that theory [we use the gauge group $\mathrm{U}(N)$ rather than $\mathrm{E}_{8}$ and we do not insist on supersymmetry] we believe that the model retains enough of the features of the heterotic model for the analysis to be instructive. Indeed the model exhibits enough interesting features to merit study in its own right.

The most general family of quasi-Kähler SU(3) structures on $\mathbb{F}_{3}$, including the standard nearly Kähler structure was considered. We have further shown how equivariant dimensional reduction over $\mathbb{F}_{3}$ can be extended to the nonKähler case and can still yield a physical particle spectrum that has many features similar to that of the Standard 
Model. We have included fermions in the analysis, albeit only in certain limited cases.

The model has yielded a remarkable vacuum structure with gauge boson, Higgs boson and fermion masses induced by the scheme, with the masses expressed as functions of the moduli of the $\mathrm{SU}(3)$ structures. The Higgs potential in particular has the exciting new feature of having vacua corresponding to solitonic solutions, opening up the possibility of Higgs masses that are inversely proportional to the gauge coupling, (4.30)-a new aspect of the Higgs mechanism that has not been noticed before.

We have also analyzed the Yukawa couplings as functions of the moduli and computed induced fermion mass matrices arising from dynamical symmetry breaking, although only in some specific cases, since the general case is technically formidable and beyond our present techniques. The particular cases, when the spinors are constant on $\mathbb{F}_{3}$, the calculation was tractable and the fermion mass matrix is given explicitly in terms of $\mathrm{SU}(3)$ moduli in (5.55).

It would be very interesting to use what we have learned from this analysis to tackle the gauge group $\mathrm{E}_{8}$ and/or a supersymmetric Lagrangian as the starting point.

\section{ACKNOWLEDGMENTS}

We thank F. Pfäffle, A. Popov, C. Sämann and C. Stephan for helpful discussions and correspondence. Part of this work was carried out while R. J. S. was visiting the Isaac Newton Institute for Mathematical Sciences in Cambridge, UK, in February/March 2012 under the auspices of the Programme "Mathematics and Applications of Branes in String and M-Theory"; he would like to thank David Berman, Neil Lambert and Sunil Mukhi for the invitation to participate and hospitality during the programme. The work of R.J.S. was supported in part by the Consolidated Grant No. ST/J000310/1 from the UK Science and Technology Facilities Council.

\section{APPENDIX A: REPRESENTATIONS OF SU(3)}

Generators and relations. - Choose a basis set $\left\{I_{A}\right\}$ for the Lie algebra su(3) with $A=1, \ldots, 8$ in such a way that $I_{7}, I_{8}$ yield a basis for the Cartan subalgebra $\mathrm{u}(1) \oplus \mathrm{u}(1)$. The structure constants $f_{A B}^{C}$ are defined by the Lie brackets

$\left[I_{A}, I_{B}\right]=f_{A B}^{C} I_{C} \quad$ with $\quad g_{A B}:=f_{A C}^{D} f_{D B}^{C}=\delta_{A B}$

where we have further chosen the basis so that it is orthonormal with respect to the Cartan-Killing form on $\operatorname{su}(3)$. Then $f_{A B C}:=f_{A B}^{D} \delta_{D C}$ is totally antisymmetric in $A, B, C$. The structure constants completely determine the geometry of the homogeneous space $\mathbb{F}_{3}$.

The nonvanishing structure constants that conform with the nearly Kähler structure of $\mathbb{F}_{3}$ and the structure equations (2.22) are given by [12]

$$
\begin{aligned}
& f_{135}=f_{425}=f_{416}=f_{326}=-\frac{1}{2 \sqrt{3}}, \\
& f_{127}=f_{347}=\frac{1}{2 \sqrt{3}} .
\end{aligned}
$$

Correspondingly, we choose the basis for $3 \times 3$ matrices of the antifundamental representation of $\operatorname{su}(3)$ given by

$I_{1}=\frac{1}{2 \sqrt{3}}\left(\begin{array}{ccc}0 & 0 & -1 \\ 0 & 0 & 0 \\ 1 & 0 & 0\end{array}\right), \quad I_{2}=\frac{1}{2 \sqrt{3}}\left(\begin{array}{ccc}0 & 0 & \mathrm{i} \\ 0 & 0 & 0 \\ \mathrm{i} & 0 & 0\end{array}\right)$,

$I_{3}=\frac{1}{2 \sqrt{3}}\left(\begin{array}{ccc}0 & 1 & 0 \\ -1 & 0 & 0 \\ 0 & 0 & 0\end{array}\right), \quad I_{4}=\frac{1}{2 \sqrt{3}}\left(\begin{array}{ccc}0 & \mathrm{i} & 0 \\ \mathrm{i} & 0 & 0 \\ 0 & 0 & 0\end{array}\right)$,

$I_{5}=\frac{1}{2 \sqrt{3}}\left(\begin{array}{ccc}0 & 0 & 0 \\ 0 & 0 & 1 \\ 0 & -1 & 0\end{array}\right), \quad I_{6}=\frac{1}{2 \sqrt{3}}\left(\begin{array}{ccc}0 & 0 & 0 \\ 0 & 0 & \mathrm{i} \\ 0 & \mathrm{i} & 0\end{array}\right)$,

$I_{7}=\frac{\mathrm{i}}{2 \sqrt{3}}\left(\begin{array}{ccc}0 & 0 & 0 \\ 0 & -1 & 0 \\ 0 & 0 & 1\end{array}\right)$ and $I_{8}=\frac{\mathrm{i}}{6}\left(\begin{array}{ccc}2 & 0 & 0 \\ 0 & -1 & 0 \\ 0 & 0 & -1\end{array}\right)$.

The matrices

$$
\begin{aligned}
& I_{1}^{-}:=\frac{1}{2}\left(I_{1}-\mathrm{i} I_{2}\right)=\frac{1}{2 \sqrt{3}}\left(\begin{array}{lll}
0 & 0 & 0 \\
0 & 0 & 0 \\
1 & 0 & 0
\end{array}\right), \\
& I_{\overline{1}}^{+}:=\frac{1}{2}\left(I_{1}+\mathrm{i} I_{2}\right)=\frac{1}{2 \sqrt{3}}\left(\begin{array}{ccc}
0 & 0 & -1 \\
0 & 0 & 0 \\
0 & 0 & 0
\end{array}\right) \text {, } \\
& I_{2}^{-}:=\frac{1}{2}\left(I_{3}-\mathrm{i} I_{4}\right)=\frac{1}{2 \sqrt{3}}\left(\begin{array}{lll}
0 & 1 & 0 \\
0 & 0 & 0 \\
0 & 0 & 0
\end{array}\right) \text {, } \\
& I_{\overline{2}}^{+}:=\frac{1}{2}\left(I_{3}+\mathrm{i} I_{4}\right)=\frac{1}{2 \sqrt{3}}\left(\begin{array}{ccc}
0 & 0 & 0 \\
-1 & 0 & 0 \\
0 & 0 & 0
\end{array}\right) \text {, } \\
& I_{3}^{-}:=\frac{1}{2}\left(I_{5}-\mathrm{i} I_{6}\right)=\frac{1}{2 \sqrt{3}}\left(\begin{array}{lll}
0 & 0 & 0 \\
0 & 0 & 1 \\
0 & 0 & 0
\end{array}\right) \text {, } \\
& I_{\overline{3}}^{+}:=\frac{1}{2}\left(I_{5}+\mathrm{i} I_{6}\right)=\frac{1}{2 \sqrt{3}}\left(\begin{array}{ccc}
0 & 0 & 0 \\
0 & 0 & 0 \\
0 & -1 & 0
\end{array}\right) \text {, } \\
& -\mathrm{i} I_{7}=\frac{1}{2 \sqrt{3}}\left(\begin{array}{ccc}
0 & 0 & 0 \\
0 & -1 & 0 \\
0 & 0 & 1
\end{array}\right) \text { and }-\mathrm{i} I_{8}=\frac{1}{6}\left(\begin{array}{ccc}
2 & 0 & 0 \\
0 & -1 & 0 \\
0 & 0 & -1
\end{array}\right)
\end{aligned}
$$


form a basis for the complexified Lie algebra $\operatorname{sl}(3, \mathbb{C})$ in the antifundamental representation. Here the complex conjugation acts by interchanging the barred and unbarred indices.

The nonvanishing structure constants $C_{A B}^{C}$ of $\operatorname{sl}(3, \mathbb{C})$ in the basis (A4) are given by

$C_{23}^{\overline{1}}=C_{31}^{\overline{2}}=C_{12}^{\overline{3}}=C_{\overline{2} \overline{3}}^{1}=C_{\overline{3} \overline{1}}^{2}=C_{\overline{1} \overline{2}}^{3}=-\frac{1}{2 \sqrt{3}}$,

$C_{71}^{1}=C_{72}^{2}=-C_{7 \overline{1}}^{\overline{1}}=-C_{7 \overline{2}}^{\overline{2}}=\frac{1}{2 \sqrt{3}}$,

$C_{73}^{3}=-C_{7 \overline{3}}^{\overline{3}}=-\frac{1}{\sqrt{3}}, \quad C_{81}^{1}=-C_{8 \overline{1}}^{\overline{1}}=-\frac{1}{2}$,

$C_{82}^{2}=-C_{8 \overline{2}}^{\overline{2}}=\frac{1}{2}, \quad C_{1 \overline{1}}^{7}=C_{2 \overline{2}}^{7}=-\frac{1}{4 \sqrt{3}}$,

$C_{3 \overline{3}}^{7}=\frac{1}{2 \sqrt{3}}, \quad C_{1 \overline{1}}^{8}=\frac{1}{4} \quad$ and $\quad C_{2 \overline{2}}^{8}=-\frac{1}{4}$.

After the rescaling (2.31), the structure constants (A5) are rescaled as

$$
\begin{aligned}
& \tilde{C}_{\beta \gamma}^{\bar{\alpha}}=2 \sqrt{3} \frac{\varsigma_{\beta} \varsigma_{\gamma}}{\varsigma_{\alpha}} C_{\beta \gamma}^{\bar{\alpha}}=-\frac{\varsigma_{\beta} \varsigma_{\gamma}}{\varsigma_{\alpha}} \varepsilon_{\beta \gamma}^{\bar{\alpha}}, \\
& \tilde{C}_{71}^{1}=C_{71}^{1}=\frac{1}{2 \sqrt{3}}, \quad \tilde{C}_{72}^{2}=C_{72}^{2}=\frac{1}{2 \sqrt{3}}, \\
& \tilde{C}_{73}^{3}=C_{73}^{3}=-\frac{1}{\sqrt{3}}, \quad \tilde{C}_{81}^{1}=C_{81}^{1}=-\frac{1}{2}, \\
& \tilde{C}_{82}^{2}=C_{82}^{2}=\frac{1}{2}, \quad \tilde{C}_{1 \overline{1}}^{7}=12 \varsigma_{1}^{2} C_{1 \overline{1}}^{7}=-\sqrt{3} \varsigma_{1}^{2}, \\
& \tilde{C}_{2 \overline{2}}^{7}=12 \varsigma_{2}^{2} C_{2 \overline{2}}^{7}=-\sqrt{3} \varsigma_{2}^{2}, \quad \tilde{C}_{3 \overline{3}}^{7}=12 \varsigma_{3}^{2} C_{3 \overline{3}}^{7}=2 \sqrt{3} \varsigma_{3}^{2}, \\
& \tilde{C}_{1 \overline{1}}^{8}=12 \varsigma_{1}^{2} C_{1 \overline{1}}^{8}=3 \varsigma_{1}^{2}, \quad \tilde{C}_{2 \overline{2}}^{8}=12 \varsigma_{2}^{2} C_{2 \overline{2}}^{8}=-3 \varsigma_{2}^{2},
\end{aligned}
$$

plus their complex conjugates. The nonvanishing structure constants $\hat{C}_{A B}^{C}$ of the Lie algebra su(3) for the complex basis of one-forms $\hat{\theta}^{\alpha}$ adapted to the Kähler structure on $\mathbb{F}_{3}$ and the structure equations (2.18) are given by

$$
\begin{aligned}
& \hat{C}_{2 \overline{3}}^{\overline{1}}=\hat{C}_{1 \overline{3}}^{\overline{2}}=-\frac{1}{2 \sqrt{6}}, \quad \hat{C}_{12}^{3}=-\frac{1}{\sqrt{6}}, \\
& \hat{C}_{1 \overline{1}}^{7}=\hat{C}_{2 \overline{2}}^{7}=\hat{C}_{3 \overline{3}}^{7}=-\frac{1}{4 \sqrt{3}}, \\
& \hat{C}_{1 \overline{1}}^{8}=\frac{1}{4} \quad \text { and } \quad \hat{C}_{2 \overline{2}}^{8}=-\frac{1}{4},
\end{aligned}
$$

and their complex conjugates, plus

$\hat{C}_{71}^{1}=\hat{C}_{72}^{2}=\frac{1}{2 \sqrt{3}}, \quad \hat{C}_{73}^{3}=-\frac{1}{\sqrt{3}}, \quad \hat{C}_{81}^{1}=-\frac{1}{2}$,

$\hat{C}_{82}^{2}=\frac{1}{2}, \quad \hat{C}_{7 \bar{\alpha}}^{\bar{\alpha}}=-\hat{C}_{7 \alpha}^{\alpha} \quad$ and $\quad \hat{C}_{8 \bar{\alpha}}^{\bar{\alpha}}=-\hat{C}_{8 \alpha}^{\alpha}$

for $\alpha=1,2,3$. Here we have chosen $R^{2}=2 \Lambda^{2}=6$.
In the basis (A4), the Chevalley generators are given by $E_{\alpha_{1}}=-2 \sqrt{3} I_{\overline{3}}^{+}, \quad E_{\alpha_{2}}=-2 \sqrt{3} I_{\overline{1}}^{+}$and $E_{\alpha_{1}+\alpha_{2}}=2 \sqrt{3} I_{2}^{-}$,

where $\alpha_{1}, \alpha_{2}$ are the simple roots of SU(3). Compared to the representations pertinent to the holomorphic Kähler loci of the moduli space [11], the change in the Chevalley generator $I_{3}^{-} \rightarrow I_{3}^{+}$corresponds to the change in sign of the almost complex structure along the $\mathbb{C} P^{1}$-fiber direction of $\mathbb{F}_{3}$.

Irreducible modules.-For each fixed pair of nonnegative integers $(k, l)$ there is an irreducible representation $\hat{V}^{k, l}$ of $\mathrm{SU}(3)$ of dimension

$$
d^{k, l}=\frac{1}{2}(k+1)(l+1)(k+l+2) .
$$

The integer $k$ is the number of fundamental representations $\hat{V}^{1,0}$ and $l$ the number of antifundamental representations $\hat{V}^{0,1}$ appearing in the usual tensor product construction of $\hat{V}^{k, l}$. All irreducible $T$ modules are one dimensional, and the collection of weight vectors of the maximal torus $T=$ $\mathrm{U}(1) \times \mathrm{U}(1)$ in the $\mathrm{SU}(3)$ label points in the weight diagram $W^{k, l}$ for $\hat{V}^{k, l}$. We denote them by $(q, m)_{n}$, where $q=$ $2 I_{z}$ and $m=3 Y$ are, respectively, isospin and hypercharge eigenvalues, and the label by the total isospin integer $n=$ $2 I$ is used to keep track of multiplicities of states in the weight diagram. They may be conveniently parametrized by a pair of independent $\mathrm{SU}(2)$ spins $j_{ \pm}$, with $2 j_{+}=$ $0,1, \ldots, k$ and $2 j_{-}=0,1, \ldots, l$, and the corresponding component spins $m_{ \pm} \in\left\{-j_{ \pm},-j_{ \pm}+1, \ldots, j_{ \pm}-1, j_{ \pm}\right\}$, which are defined in terms of Young tableaux as follows. Represent the irreducible $T$ module $V_{(q, m)_{n}}$ with weight vector $(q, m)_{n}=(1,1)_{1}$ by $\otimes$, that with $(q, m)_{n}=$ $(-1,1)_{1}$ by $\square$, and that with $(q, m)_{n}=(0,-2)_{0}$ by $\square$. Then the $\mathrm{SU}(3) \rightarrow \mathrm{U}(1) \times \mathrm{U}(1)$ decomposition of the fundamental representation

$$
\left.\hat{V}^{1,0}\right|_{T}=V_{(1,1)_{1}} \oplus V_{(-1,1)_{1}} \oplus V_{(0,-2)_{0}}
$$

is depicted by

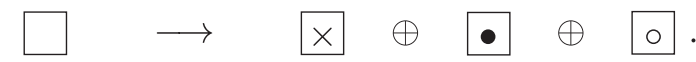

In terms of the SU(3) Young tableaux, the irreducible representation $\hat{V}^{k, l}$ corresponds to the diagram

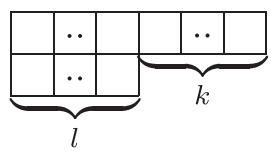

and this contains all $\mathrm{U}(1) \times \mathrm{U}(1)$ representations 


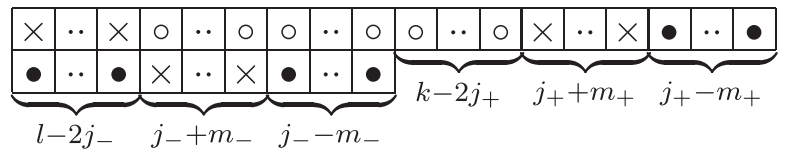

of dimension $2 j_{+}+2 j_{-}+1$, isospin charge $2 m_{+}+2 m_{-}$, and hypercharge $2(l-k)+6\left(j_{+}-j_{-}\right)$, with multiplicity one. This gives

$q=2\left(m_{+}+m_{-}\right)$,

$m=6\left(j_{+}-j_{-}\right)-2(k-l)$ and $n=2\left(j_{+}+j_{-}\right)$.

The $\mathrm{SU}(2)$ spin $j_{+}\left(j_{-}\right)$is the value of the isospin contributed by the upper (lower) indices of the SU(3) tensor corresponding to the irreducible module $\hat{V}^{k, l}$. The integers $(q, m)_{n}$ all have the same even/odd parity.

Biedenharn basis.-To explicitly represent the coset generators of $\mathbb{F}_{3}$, we use the Biedenharn basis for the irreducible representation $\hat{V}^{k, l}$ of SU(3) [11]. The generators of the complex torus $T^{\mathbb{C}}=\mathbb{C}^{*} \times \mathbb{C}^{*}$ for the irreducible module corresponding to the weight vector $(q, m)_{n}$ in this basis are given by

$$
-\mathrm{i} I_{7}^{(q, m)_{n}}=\frac{q}{2 \sqrt{3}} \quad \text { and } \quad-\mathrm{i} I_{8}^{(q, m)_{n}}=\frac{m}{6}
$$

while the nonvanishing off-diagonal matrix elements of the remaining generators of $\operatorname{SL}(3, \mathbb{C})$ are

$$
\begin{aligned}
I_{1}^{-(q, m)_{n},(q-1, m+3)_{n \pm 1}} & =\sqrt{\frac{n \mp q+1 \pm 1}{24(n+1)}} \lambda_{k, l}^{ \pm}(n, m), \\
I_{2}^{-(q, m)_{n},(q-1, m-3)_{n \pm 1}} & =\sqrt{\frac{n \mp q+1 \pm 1}{24(n+1 \pm 1)}} \lambda_{k, l}^{\mp}(n \pm 1, m-3), \\
I_{3}^{-(q, m)_{n},(q+2, m)_{n}} & =\sqrt{\frac{(n-q)(n+q+2)}{48}},
\end{aligned}
$$

where

$$
\begin{aligned}
& \lambda_{k, l}^{+}(n, m)=\frac{1}{\sqrt{n+2}} \sqrt{\left(\frac{k+2 l}{3}+\frac{n}{2}+\frac{m}{6}+2\right)\left(\frac{k-l}{3}+\frac{n}{2}+\frac{m}{6}+1\right)\left(\frac{2 k+l}{3}-\frac{n}{2}-\frac{m}{6}\right)}, \\
& \lambda_{k, l}^{-}(n, m)=\frac{1}{\sqrt{n}} \sqrt{\left(\frac{k+2 l}{3}-\frac{n}{2}+\frac{m}{6}+1\right)\left(\frac{l-k}{3}+\frac{n}{2}-\frac{m}{6}\right)\left(\frac{2 k+l}{3}+\frac{n}{2}-\frac{m}{6}+1\right) .}
\end{aligned}
$$

The latter constants are defined for $n>0$, and we set $\lambda_{k, l}^{-}(0, m):=0$. The analogous relations for $I_{\bar{\alpha}}^{+}$can be derived by Hermitian conjugation of (A17) using the property $\left(I_{\bar{\alpha}}^{+}\right)^{\dagger}=-I_{\alpha}^{-}$.

\section{APPENDIX B: MATRIX ELEMENTS OF INVARIANT CURVATURES}

The diagonal matrix elements of the curvature (3.9) of the gauge potential (3.9) at each vertex $(q, m)_{n} \in W^{k, l}$ of the weight diagram for $\hat{V}^{k, l}$ can be computed by substituting (A17) and are given by

$$
\begin{aligned}
& \mathcal{F}^{(q, m)_{n}(q, m)_{n}}=F^{(q, m)_{n}}+\frac{\varsigma_{1}^{2}}{2} \tilde{\Theta}^{1} \wedge \tilde{\Theta}^{\overline{1}} \sum_{ \pm}\left[\frac{n \mp q+1 \pm 1}{n+1} \lambda_{k, l}^{ \pm}(n, m)^{2}\left(\mathbf{1}_{N_{(q, m)_{n}}}-\phi_{(q, m)_{n}}^{1}{ }^{( \pm) \dagger} \phi_{(q, m)_{n}}^{1}{ }^{( \pm)}\right)\right. \\
& \left.-\frac{n \mp q+1 \mp 1}{n+1 \mp 1} \lambda_{k, l}^{ \pm}(n \mp 1, m-3)^{2}\left(\mathbf{1}_{N_{(q, m)_{n}}}-\phi_{(q+1, m-3)_{n \mp 1}}^{1}{ }^{( \pm)} \phi_{(q+1, m-3)_{n \mp 1}}^{1}{ }^{( \pm) \dagger}\right)\right] \\
& +\frac{\varsigma_{2}^{2}}{2} \tilde{\Theta}^{2} \wedge \tilde{\Theta}^{\overline{2}} \sum_{ \pm}\left[\frac{n \mp q+1 \pm 1}{n+1 \pm 1} \lambda_{k, l}^{\mp}(n \pm 1, m-3)^{2}\left(\mathbf{1}_{N_{(q, m)_{n}}}-\phi_{(q, m)_{n}}^{2}{ }^{( \pm) \dagger} \phi_{(q, m)_{n}}^{2}{ }^{( \pm)}\right)\right. \\
& \left.-\frac{n \mp q+1 \mp 1}{n} \lambda_{k, l}^{\mp}(n, m)^{2}\left(\mathbf{1}_{N_{(q, m) n}}-\phi_{(q+1, m+3)_{n \mp 1}}^{2}{ }^{( \pm)} \phi_{(q+1, m+3)_{n+1}}^{2}{ }^{( \pm) \dagger}\right)\right] \\
& +\frac{\varsigma_{3}^{2}}{4} \tilde{\Theta}^{3} \wedge \tilde{\Theta}^{\overline{3}}\left[(n-q)(n+q+2)\left(\mathbf{1}_{N_{(q, m)_{n}}}-\phi_{(q, m)_{n}}^{3}{ }^{\dagger} \phi_{(q, m)_{n}}^{3}\right)\right. \\
& \left.-(n+q)(n-q+2)\left(\mathbf{1}_{N_{(q, m) n}}-\phi_{(q-2, m)_{n}}^{3} \phi_{(q-2, m)_{n}}^{3}{ }^{\dagger}\right)\right] \text {, }
\end{aligned}
$$

where $F^{(q, m)_{n}}=\mathrm{d} A^{(q, m)_{n}}+A^{(q, m)_{n}} \wedge A^{(q, m)_{n}}$ is the curvature of the vector bundle $E_{(q, m)_{n}} \rightarrow M$, and we suppress tensor products to simplify the notation. The remaining nonvanishing off-diagonal matrix elements of the curvature two-form $\mathcal{F}$ are given by 


$$
\begin{aligned}
& \mathcal{F}^{(q-1, m+3)_{n \pm 1}(q, m)_{n}}=\lambda_{k, l}^{ \pm}(n, m)\left\{\varsigma_{1} \sqrt{\frac{(n \mp q+1 \pm 1)}{2(n+1)}} \tilde{\Theta}^{\overline{1}} \wedge D \phi_{(q, m)_{n}}^{1}{ }^{( \pm)}+\frac{\varsigma_{2} \varsigma_{3}}{2} \tilde{\Theta}^{2}\right. \\
& \wedge \tilde{\Theta}^{3}\left[\sqrt{\frac{\left((n+1 \pm 1)^{2}-q^{2}\right)(n \pm q+1 \pm 1)}{2(n+1)}}\left(\phi_{(q, m) n}^{1}{ }^{( \pm)}-\phi_{(q-1, m+3)_{n \pm 1}}^{3}{ }^{\dagger} \phi_{(q+1, m+3)_{n \pm 1}}^{2}{ }^{(\mp) \dagger}\right)\right. \\
& \left.\left.-\sqrt{\frac{(n \pm q+1 \mp 1)(n-q+2)(n+q)}{2(n+1)}}\left(\phi_{(q, m)_{n}}^{1}{ }^{( \pm)}-\phi_{(q-1, m+3)_{n \pm 1}}^{2}{ }^{(\mp) \dagger} \phi_{(q-2, m)_{n}}^{3}\right)\right]\right\} \text {, } \\
& \mathcal{F}^{(q-1, m-3)_{n \pm 1}(q, m)_{n}}=\lambda_{k, l}^{\mp}(n \pm 1, m-3)\left\{\varsigma_{2} \sqrt{\frac{(n \mp q+1 \pm 1)}{2(n+1 \pm 1)}} \tilde{\Theta}^{\overline{2}} \wedge D \phi_{(q, m)_{n}}^{2}{ }^{( \pm)}+\frac{\varsigma_{3} \varsigma_{1}}{2} \tilde{\Theta}^{3}\right. \\
& \wedge \tilde{\Theta}^{1}\left[\sqrt{\frac{\left((n+1 \pm 1)^{2}-q^{2}\right)(n \pm q+1 \pm 1)}{2(n+1 \pm 1)}}\left(\phi_{(q, m)_{n}}^{2}{ }^{( \pm)}-\phi_{(q-1, m-3)_{n \pm 1}}^{1}{ }^{(\mp) \dagger} \phi_{(q-2, m)_{n}}^{3}\right)\right. \\
& \left.-\sqrt{\frac{(n \pm q+1 \mp 1)(n-q+2)(n+q)}{2(n+1 \pm 1)}}\left(\phi_{(q, m)_{n}}^{2}{ }^{( \pm)}-\phi_{(q-1, m-3)_{n \pm 1}}^{3} \phi_{(q+1, m-3)_{n \pm 1}}^{(\mp) \dagger)}\right]\right\} \text {, } \\
& \mathcal{F}^{(q+2, m)_{n}(q, m)_{n}}=\frac{\varsigma_{3}}{2} \sqrt{(n-q)(n+q+2)} \tilde{\Theta}^{\overline{3}} \wedge D \phi_{(q, m)_{n}}^{3}+\frac{\boldsymbol{\varsigma}_{1} \boldsymbol{\varsigma}_{2}}{2} \sqrt{(n+1)^{2}-(q+1)^{2}} \tilde{\Theta}^{1} \\
& \wedge \tilde{\Theta}^{2} \sum_{ \pm}\left[\lambda_{k, l}^{ \pm}(n \mp 1, m-3)^{2}\left(\phi_{(q, m)_{n}}^{3}-\phi_{(q+2, m)_{n}}^{2}{ }^{(\mp) \dagger} \phi_{(q+1, m-3)_{n \mp 1}}^{1}{ }^{( \pm) \dagger}\right)\right. \\
& \left.-\lambda_{k, l}^{ \pm}(n, m)^{2}\left(\phi_{(q, m)_{n}}^{3}-\phi_{(q+2, m)_{n}}^{1}( \pm) \dagger \phi_{(q+1, m+3)_{n \pm 1}}^{2}(\mp) \dagger\right)\right] \text {, }
\end{aligned}
$$

and

$$
\begin{aligned}
& \mathcal{F}^{(q+2, m)_{n}(q-1, m+3)_{n \pm 1}}=\frac{\varsigma_{1} \varsigma_{3}}{2} \sqrt{\frac{(n-q)(n+q+2)(n \mp q+1 \pm 1)}{2(n+1)}} \lambda_{k, l}^{ \pm}(n, m) \tilde{\Theta}^{1} \wedge \tilde{\Theta}^{\overline{3}}\left(\phi_{(q, m)_{n}}^{3} \phi_{(q, m)_{n}}^{1}{ }^{( \pm) \dagger}\right. \\
& \left.-\phi_{(q+2, m)_{n}}^{1}( \pm) \dagger \phi_{(q-1, m+3)_{n \pm 1}}^{3}\right), \\
& \mathcal{F}^{(q+2, m)_{n}(q-1, m-3)_{n \pm 1}}=\frac{\varsigma_{2} \varsigma_{3}}{2} \sqrt{\frac{(n-q)(n+q+2)(n \mp q+1 \pm 1)}{2(n+1 \pm 1)}} \lambda_{k, l}^{\mp}(n \pm 1, m-3) \tilde{\Theta}^{2} \wedge \tilde{\Theta}^{\overline{3}}\left(\phi_{(q, m)_{n}}^{3} \phi_{(q, m)_{n}}^{2}{ }^{( \pm) \dagger}\right. \\
& \left.-\phi_{(q+2, m)_{n}}^{2}{ }^{( \pm) \dagger} \phi_{(q-1, m-3)_{n \pm 1}}^{3}\right), \\
& \mathcal{F}^{(q+1, m-3)_{n \mp 1}(q+1, m+3)_{n \pm 1}}=\frac{\varsigma_{1} \varsigma_{2}}{2} \sqrt{\frac{\left((n+1)^{2}-(q+1)^{2}\right)}{(n+1 \mp 1)(n+1)}} \lambda_{k, l}^{ \pm}(n, m) \lambda_{k, l}^{ \pm}(n \mp 1, m-3) \tilde{\Theta}^{1} \wedge \tilde{\Theta}^{\overline{2}}\left(\phi_{(q+2, m)_{n}}^{2}{ }^{(\mp)} \phi_{(q+2, m)_{n}}^{1}{ }^{( \pm) \dagger}\right.
\end{aligned}
$$

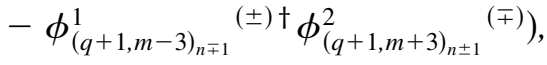

plus their hermitIan conjugates $\mathcal{F}^{\left(q^{\prime}, m^{\prime}\right)_{n^{\prime}}(q, m)_{n}}=-\left(\mathcal{F}^{(q, m)_{n}\left(q^{\prime}, m^{\prime}\right)_{n^{\prime}}}\right)^{\dagger}$ for $\left(q^{\prime}, m^{\prime}\right)_{n^{\prime}} \neq(q, m)_{n}$. Here

$$
\begin{aligned}
D \phi_{(q, m)_{n}}^{( \pm)} & =\mathrm{d} \phi_{(q, m)_{n}}^{1}{ }^{( \pm)}+A^{(q-1, m+3)_{n \pm 1}} \phi_{(q, m)_{n}}^{1}{ }^{( \pm)}-\phi_{(q, m)_{n}}^{1}{ }^{( \pm)} A^{(q, m)_{n},}, \\
D \phi_{(q, m)_{n}}^{2}{ }^{( \pm)} & =\mathrm{d} \phi_{(q, m)_{n}}^{2}{ }^{( \pm)}+A^{(q-1, m-3)_{n \pm 1}} \phi_{(q, m)_{n}}^{2}{ }^{( \pm)}-\phi_{(q, m)_{n}}^{2}{ }^{( \pm)} A^{(q, m)_{n}}, \\
D \phi_{(q, m)_{n}}^{3} & =\mathrm{d} \phi_{(q, m)_{n}}^{3}+A^{(q+2, m)_{n}} \phi_{(q, m)_{n}}^{3}-\phi_{(q, m)_{n}}^{3} A^{(q, m)_{n},}
\end{aligned}
$$

are bifundamental covariant derivatives of the Higgs fields on $M$. 
[1] G. L. Cardoso, G. Curio, G. Dall'Agata, D. Lüst, P. Manousselis, and G. Zoupanos, Nucl. Phys. B652, 5 (2003).

[2] A. Strominger, Nucl. Phys. B274, 253 (1986).

[3] J. Scherk and J. H. Schwarz, Nucl. Phys. B153, 61 (1979).

[4] D. Kapetanakis and G. Zoupanos, Phys. Rep. 219, 4 (1992).

[5] A. Chatzistavrakidis and G. Zoupanos, J. High Energy Phys. 09 (2009) 077.

[6] M. Klaput, A. Lukas, and C. Matti, J. High Energy Phys. 09 (2011) 100.

[7] D. Lüst, Nucl. Phys. B276, 220 (1986).

[8] O. Lechtenfeld, C. Nölle, and A. D. Popov, J. High Energy Phys. 09 (2010) 074.

[9] N. Irges and G. Zoupanos, Phys. Lett. B 698, 146 (2011).

[10] O. Lechtenfeld, A. D. Popov, and R. J. Szabo, Prog. Theor. Phys. Suppl. 171, 258 (2007); B. P. Dolan and R. J. Szabo, Gen. Relativ. Gravit. 43, 2453 (2011).

[11] O. Lechtenfeld, A. D. Popov, and R. J. Szabo, J. High Energy Phys. 08 (2008) 093.

[12] A. D. Popov and R. J. Szabo, J. High Energy Phys. 02 (2012) 033.
[13] B. P. Dolan and R. J. Szabo, J. High Energy Phys. 03 (2009) 059.

[14] B. P. Dolan and R. J. Szabo, J. High Energy Phys. 08 (2009) 038.

[15] R. Bott and L.W. Tu, Differential Forms in Algebraic Topology (Springer, New York, 1982).

[16] S. Chiossi and S. M. Salamon, in Differential Geometry, Valencia 2001, edited by O. Gil-Medrano and V. Miquel (World Scientific, Singapore, 2002), pp. 115-133.

[17] I. Agricola, Commun. Math. Phys. 232, 535 (2003).

[18] T. Houri, D. Kubizňák, C. Warnick, and Y. Yasui, Classical Quantum Gravity 27, 185019 (2010).

[19] B. P. Dolan, J. High Energy Phys. 05 (2003) 018.

[20] G. D. Landweber, Represent. Theor. 4, 466 (2000).

[21] T. Kimura and P. Yi, J. High Energy Phys. 07 (2006) 030.

[22] J.-M. Bismut, Math. Ann. 284, 681 (1989).

[23] T. Kimura, J. High Energy Phys. 08 (2007) 048.

[24] R. Rajaraman, Solitons and Instantons (North-Holland, Amsterdam, 1982).

[25] T. Friedrich and S. Ivanov, Asian J. Math. 6, 303 (2002). 